\title{
Water-Quality Data From Five Oregon Stream Basins
}
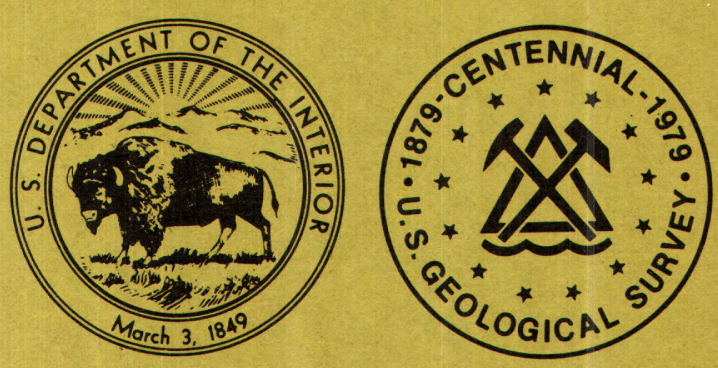

Prepared in cooperation with the OREGON DEPARTMENT OF ENVIRONMENTAL QUALITY 
WATER-QUALITY DATA FROM

FIVE OREGON STREAM BASINS

By Timothy L. Miller

U.S. GEOLOGICAL SURVEY

Open-File Report 79-1535

Prepared in cooperation with the

OREGON DEPARTMENT OF ENVIRONMENTAL QUALITY

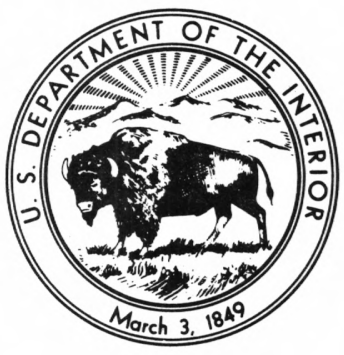




\section{UNITED STATES DEPARTMENT OF THE INTERIOR \\ CECIL D. ANDRUS, Secretary \\ GEOLOGICAL SURVEY}

H. William Menard, Director

For additional information write to:

U.S. GEOLOGICAL SURVEY

P.O. Box 3202

Portland, Oregon 97208 
Abstract-1- 1

Introduction-

Sampling-1.-

Site selection--

Analyses and equipment-_. 2

Physica1 characteristics-1

Chemical and miscellaneous data-

Periphyton-- 3

Benthic invertebrates- 3

Hydrologic data- 3

References

\section{ILLUSTRATIONS}

Figure 1. Map showing five Oregon stream basins and their sampling

Page

sites-..-...- 5

2-6. Map showing sampling sites in:

2. Evans Creek basin- 7

3. Mola1la River basin-_ 8

4. Siuslaw Basin area-_. 9

5. South Fork John Day River basin-_... 10

6. Umatilla River basin-... 11

TABLES

Page

Conversion factors-1v

Table 1. Locations of sampling sites-1 6

2. Physical characteristics-_. 12

3. Chemical data-- 38

4. Miscellaneous data-_... 43

5. Taxa and numbers of periphyton-_. 48

6. Taxa and numbers of benthic invertebrates... 74 


\section{Conversion factors for inch-pound system and International System Units (SI)}

[For use of those readers who may prefer to use metric units rather than inch-pound units, the conversion factors for the terms used in this report are listed below:]

\begin{tabular}{lcl}
\hline Multiply inch-pound units & By & To obtain metric unit \\
\hline Length & \\
\hline $\begin{array}{l}\text { inch (in) } \\
\text { foot (ft) } \\
\text { mile (mi) }\end{array}$ & 25.40 & $\begin{array}{l}\text { millimeter }(\mathrm{mm}) \\
\text { meter }(\mathrm{m}) \\
\text { kilometer }(\mathrm{km})\end{array}$ \\
\hline & 0.3048 & \\
\hline square inch $\left(\mathrm{in}^{2}\right)$ & 1.609 & Area \\
square foot $\left(\mathrm{ft}^{2}\right)$ & 6.452 & $\begin{array}{l}\text { square centimeter }\left(\mathrm{cm}^{2}\right) \\
\text { square meter }\left(\mathrm{m}^{2}\right)\end{array}$ \\
\hline & 0.09290 & \\
\hline cubic foot per second $\left(\mathrm{ft}^{3} / \mathrm{s}\right)$ & Specific combination & cubic meter per second \\
& 0.0283 & $\left(\mathrm{~m}^{3} / \mathrm{s}\right)$ \\
\hline
\end{tabular}

$\underline{1} / \mathrm{Temp}^{\circ} \mathrm{C}=\left(\mathrm{temp}^{\circ} \mathrm{F}-32\right) 1.8$. 


\title{
WATER-QUALITY DATA FROM FIVE OREGON STREAM BASINS
}

\author{
By Timothy L. Miller
}

\section{ABSTRACT}

The U.S. Geological Survey collected water-quality data in five Oregon stream basins during summer low-flow conditions in 1977 and 1978. During the two sampling periods, a total of 18 different sites were sampled. Several sites were sampled twice in 1977, and some sites were sampled in both 1977 and 1978. Included in the sampling were diel trace of dissolved oxygen, temperature, specific conductance, $\mathrm{pH}$, and solar radiation. In addition, periphyton and benthic invertebrate samples were collected and identified.

\section{INTRODUCTION}

The U.S. Geological Survey (USGS), in cooperation with the Oregon Department of Environmental Quality (DEQ), collected water-quality data from five Oregon stream basins (fig. 1). Data collected by the USGS provided waterquality information on drought (1977) and normal low-flow (1978) conditions. The DEQ collected data that included (1) terrain characteristics, (2) landmanagement practices, and (3) resultant channel quality as determined by channel morphology and fish habitat (Rickert and others, 1978, p. iii). The DEQ data for Evans Creek basin are presented in the report by Rickert and others. Data for the four other basins are contained in several additional $\mathrm{DEQ}$ reports.

The five basins represent different climates, geology, and land uses that are characteristic of the main geographical regions and physiographic divisions of the State. These basins encompass a broad range of conditions that influence nonpoint sources of pollution outside major urban areas. 


\section{Site Selection}

Locations of sampling sites for each basin are shown on figures 2 through 6 and are listed in table 1. Sampling sites were selected to represent drainage areas with different land uses or different intensities of a particular land use. A11 sampling sites were upstream from any known point sources, and none of the sites was downstream from reservoirs. The number of sites in each basin depended on the size of the basin and the different land uses in that basin. Data were collected at several sites in 1977 during very dry summer conditions and again in 1978 during near-normal low-flow conditions. Al1 data were collected during low-flow steady-state conditions. Steady-state conditions were assumed, for each site, when no significant precipitation had occurred in the 10- to 14-day period prior to sampling.

\section{Analyses and Equipment}

The data collected have been grouped into four categories for each sampling period and are presented in tables 2 to 6 . The four categories are (1) physical characteristics, (2) chemical and miscellaneous data, (3) periphyton, and (4) benthic invertebrates. Analyses and equipment for each category will be discussed separately.

\section{Physical Characteristics}

Field measurements of temperature, specific conductance, dissolved oxygen, and $\mathrm{pH}$ were made with a Martek / multiparameter monitor which was linked to an Elnik strip chart recorder. Solar radiation was measured with a Weathermeasure Star Pyranometer. At each site, the Martek probe was placed in a riffle area. The Martek equipment was calibrated three or more times during a 24-hour period. The pyranometer was calibrated at the time it was linked to the Elnik recorder. For this report, the continuous record for each characteristic was reduced to a time-weighted average hourly value.

\section{Chemica1 and Miscellaneous Data}

Samples for analysis of major ions, nutrients, and alkalinity were collected, preserved, and analyzed by methods outlined by Brown and others (1970). Discharge was measured by standard USGS techniques (Buchanan and Somers, 1969). Suspended sediment was collected and analyzed for concentration according to methods described by Guy (1969). Greeson and others (1977) provided methods for collection and analysis of algal-growth potential and fecal coliform, and for the oxygen light-dark bottle test for phytoplankton. Samples for periphyton biomass and chlorophyll were collected by the same procedure described in the section entitled "Periphyton." After collection, the

\footnotetext{
- The use of brand names in this report is for identification purposes only and does not imply endorsement by the U.S. Geological Survey.
} 
biomass and water mixtures for both analyses were filtered through a Gelman glass-fiber filter type A-E. The biomass filters were air-dried, whereas the chlorophyl1 filters were placed on ice. The chlorophyll samples were analyzed by the USGS laboratory in Atlanta, Ga. The biomass filters were dried at $105^{\circ} \mathrm{C}$ to determine dry weight and were then ignited at $500^{\circ} \mathrm{C}$ for 1 hour to determine the dry weight less ash weight.

\section{Periphyton}

Samples were collected for periphyton identification, biomass, and chlorophy11 by selecting 10 rocks from a riffle area near the Martek probe. The rocks were selected to represent the entire cross section on a flowweighted basis because more rocks were from the deeper riffle areas than from the shallower bank areas. Where the riffle areas were sma11 or the rocks were few, only five rocks were selected. An area 1 inch square on each rock was scraped using a glass slide. Care was taken to scrape periphyton from only the upper area of the rock which was exposed to sunlight.

\section{Benthic Invertebrates}

Benthic invertebrate samples were collected from riffle areas by using a Surber sampler covering 1 square foot. For most sites, only one sample was collected, generally from midchannel near the center of the riffle area. At some sites where the riffle area was relatively large compared to the other sites, or if the number of organisms was relatively sma11, more than one sample was collected and composited.

\section{HYDROLOGIC DATA}

Data collected for the study are listed in the tables that follow. Each table is organized in sequential site-number order. Table 2 contains the average hourly values of the physical characteristics. Table 3 shows the results of the chemical analyses. Table 4 includes several additional biological and miscellaneous tests. Tables 5 and 6 contain identification and abundance of periphyton and benthic invertebrates, respectively. 
Brown, Eugene, Skougstad, M. W., and Fishman, M. J., 1970, Methods for co1lection and analysis of water samples for dissolved minerals and gases: U.S. Geologica1 Survey Techniques of Water-Resources Investigations, book 5, chap. A1, 160 p.

Buchanan, T. J., and Somers, W. P., 1969, Discharge measurements at gaging stations: U.S. Geological Survey Techniques of Water-Resources Investigations, book 3, chap. A8, 65 p.

Greeson, P. E., Ehlke, T. A., Irwin, G. A., Lium, B. W., and Slack, K. V., 1977, Methods for collection and analysis of aquatic biological and microbiologica1 samples: U.S. Geologica1 Survey Techniques of WaterResources Investigations, book 5, chap。A4, 332 p.

Guy, H. P., 1969, Laboratory theory and methods for sediment analysis: U.S. Geologica1 Survey Techniques of Water-Resources Investigations, book 5, chap. $\mathrm{C} 1,58 \mathrm{p}$.

Rickert, D。 A., Beach, G. L., Jackson, J. E。, Anderson, D。 M., Hazen, H. H., and Suwijn, E., 1978, Oregon's procedure for assessing the impacts of land management activities on erosion related non-point source problems: Oregon Department of Environmental Quality, 219 p. 


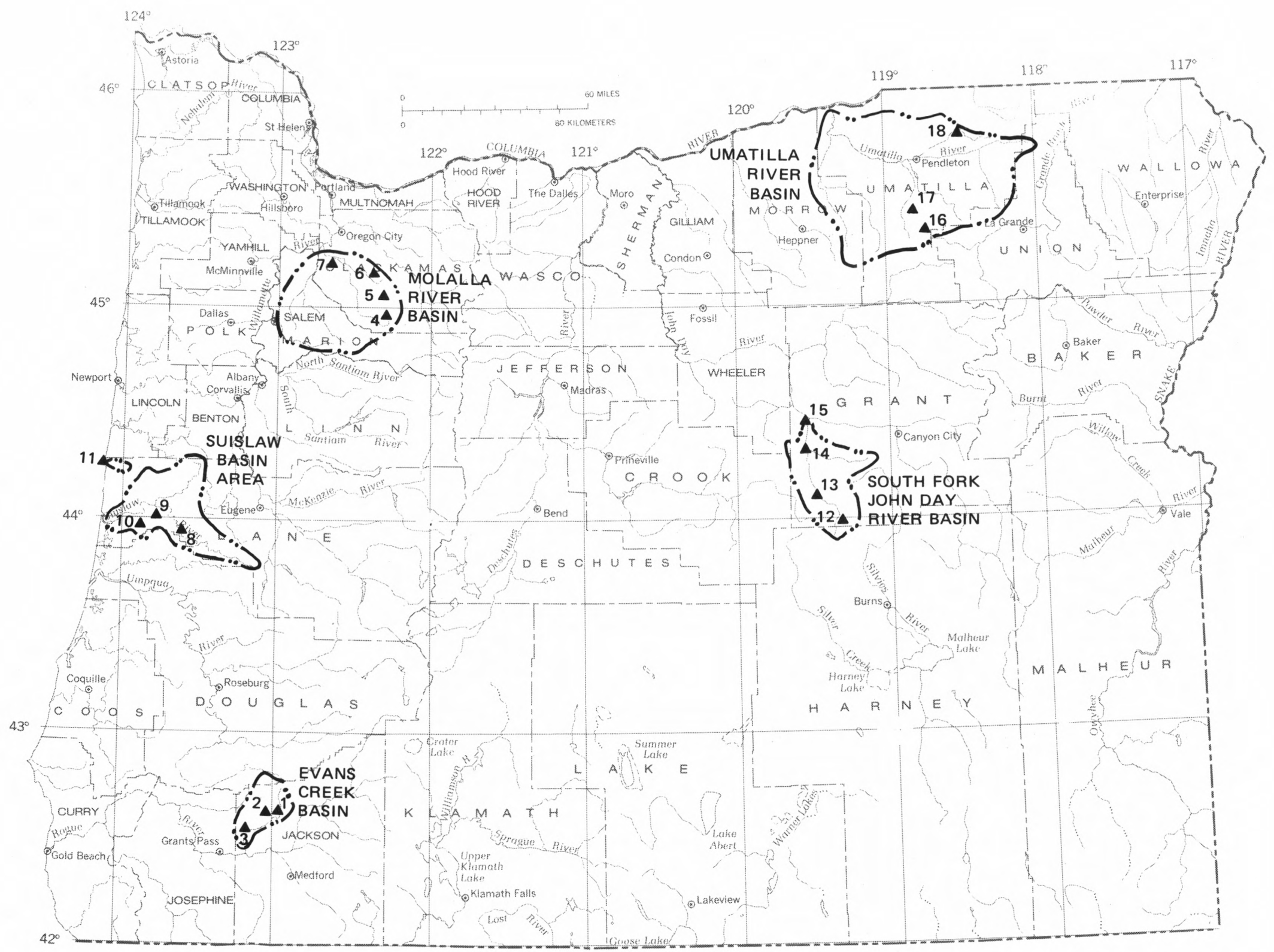

Figure 1.-Five Oregon stream basins and their sampling sites. 


\begin{tabular}{|c|c|c|c|}
\hline $\begin{array}{l}\text { Site } \\
\text { No. }\end{array}$ & Site name & Location & $\begin{array}{l}\text { Land use upstream from } \\
\text { sampling site }\end{array}$ \\
\hline $1 \mathrm{~A}$ & Evans Creek above West Fork & 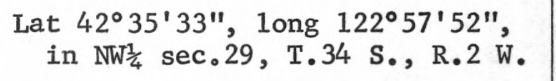 & Forest (active logging). \\
\hline $1 \mathrm{~B}$ & Evans Creek above West Fork & 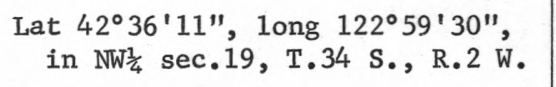 & Do. \\
\hline 2 & West Fork Evans Creek & 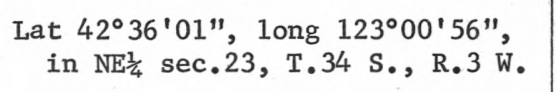 & $\begin{array}{l}\text { Forest (active logging), } \\
\text { native grazing. }\end{array}$ \\
\hline 3 & Evans Creek at Minthorne Road & 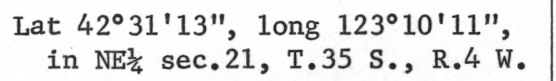 & $\begin{array}{l}\text { Forest, pasture, irrigation } \\
\text { withdrawa1. }\end{array}$ \\
\hline 4 & Lost Créek, Mola1la River basin & $\begin{array}{l}\text { Lat } 44^{\circ} 59^{\prime} 12^{\prime \prime} \text {, long } 122^{\circ} 15^{\prime} 58^{\prime \prime} \text {, } \\
\text { in SW } \frac{1}{4} \text { sec.1, T.7 S., R.4 E。 }\end{array}$ & Forest (active logging). \\
\hline 5 & $\begin{array}{l}\text { Dead Horse Canyon Creek, Molalla River } \\
\text { basin }\end{array}$ & $\begin{array}{l}\text { Lat } 45^{\circ} 05^{\prime} 10^{\prime \prime} \text {, long } 122^{\circ} 19^{\prime} 06^{\prime \prime} \text {, } \\
\text { in SE } \frac{1}{4} \text { sec. } 33 \text {, T. } 5 \text { S., R.4 E。 }\end{array}$ & Do. \\
\hline 6 & Dorn Creek, Molalla River basin & $\begin{array}{l}\text { Lat } 45^{\circ} 12^{\prime} 28^{\prime \prime} \text {, long } 122^{\circ} 21^{\prime} 52^{\prime \prime} \text {, } \\
\text { in SW } \frac{1}{4} \text { sec.19, T.4 S., R.4 E. }\end{array}$ & Forest. \\
\hline 7 & Milk Creek, Mola1la River basin & $\begin{array}{l}\text { Lat } 45^{\circ} 14^{\prime} 22^{\prime \prime} \text {, long } 122^{\circ} 39^{\prime} 14^{\prime \prime} \text {, } \\
\text { in NW } \frac{1}{4} \text { sec. } 11, \text { T.4 S., R.1 E. }\end{array}$ & $\begin{array}{l}\text { Forest, irrigation with- } \\
\text { drawa1, pasture. }\end{array}$ \\
\hline 8 & Wo1f Creek, Siuslaw River basin & 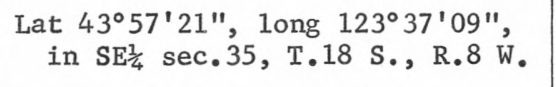 & Do. \\
\hline 9 & Knowles Creek, Siuslaw River basin & $\begin{array}{l}\text { Lat } 44^{\circ} 00^{\prime} 51^{\prime \prime}, \text { long } 123^{\circ} 47^{\prime} 10^{\prime \prime} \text {, } \\
\text { in SW } \frac{1}{4} \text { sec.9, T.18 S., R.9 W. }\end{array}$ & Forest (active logging). \\
\hline 10 & Cedar Creek, Siuslaw River basin & $\begin{array}{l}\text { Lat } 43^{\circ} 58^{\prime} 32^{\prime \prime}, \text { long } 123^{\circ} 54^{\prime} 24^{\prime \prime}, \\
\quad \text { in SW } \frac{1}{4} \text { sec. } 28, \text { T.18 S., R.10 W. }\end{array}$ & Do. \\
\hline 11 & Bob Creek, Siuslaw Basin $1 /$ & 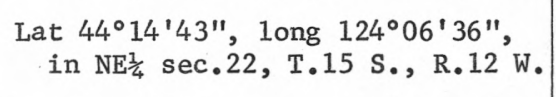 & Forest. \\
\hline 12 & $\begin{array}{l}\text { South Fork John Day River above } \\
\text { Forest Service boundary }\end{array}$ & 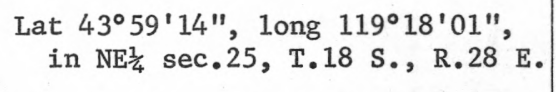 & Do. \\
\hline 13 & $\begin{array}{l}\text { South Fork John Day River above } \\
\text { Little Pine Creek }\end{array}$ & $\begin{array}{l}\text { Lat } 44^{\circ} 07^{\prime} 20^{\prime \prime}, \text { long } 119^{\circ} 29^{\prime} 28^{\prime \prime} \text {, } \\
\quad \text { in SW } \frac{1}{4} \text { sec.4, T.17 S., R.27 E. }\end{array}$ & $\begin{array}{l}\text { Forest (active logging), } \\
\text { pasture. }\end{array}$ \\
\hline 14 & $\begin{array}{l}\text { B1ack Canyon Creek, South Fork John } \\
\text { Day River basin }\end{array}$ & 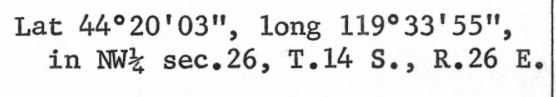 & Forest, native grazing. \\
\hline 15 & South Fork John Day River at Dayville & 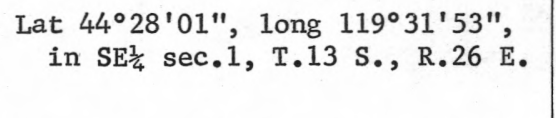 & $\begin{array}{l}\text { Urbanization, irrigation } \\
\text { withdrawa1, pasture, } \\
\text { forest, native grazing. }\end{array}$ \\
\hline 16 & Pearson Creek, Umatilla River basin & $\begin{array}{l}\text { Lat } 45^{\circ} 20^{\prime} 15^{\prime \prime} \text {, long } 118^{\circ} 43^{\prime} 08^{\prime \prime}, \\
\text { in SE } \frac{1}{4} \text { sec.4, T.3 S., R.33 E. }\end{array}$ & Forest (active logging). \\
\hline 17 & $\begin{array}{l}\text { East Fork Birch Creek, Umatilla River } \\
\text { basin }\end{array}$ & $\begin{array}{l}\text { Lat } 45^{\circ} 25^{\prime} 54^{\prime \prime}, \text { long } 118^{\circ} 49^{\prime} 13^{\prime \prime} \text {, } \\
\text { in } \operatorname{SE}_{\frac{1}{4}} \text { sec. } 4, \text { T.1 S., R.32 E. }\end{array}$ & $\begin{array}{l}\text { Forest, pasture, native } \\
\text { grazing. }\end{array}$ \\
\hline 18 & Wild Horse Creek, Umatilla River basin & $\begin{array}{l}\text { Lat } 45^{\circ} 47^{\prime} 46^{\prime \prime}, \text { long } 118^{\circ} 28^{\prime} 28^{\prime \prime} \text {, } \\
\text { in SW } \frac{1}{4} \text { sec.29, T.4 N., R.35 E. }\end{array}$ & $\begin{array}{l}\text { Pasture, native grazing, } \\
\text { annual crop agriculture. }\end{array}$ \\
\hline
\end{tabular}

1/ Bob Creek drains directly into the Pacific Ocean。 


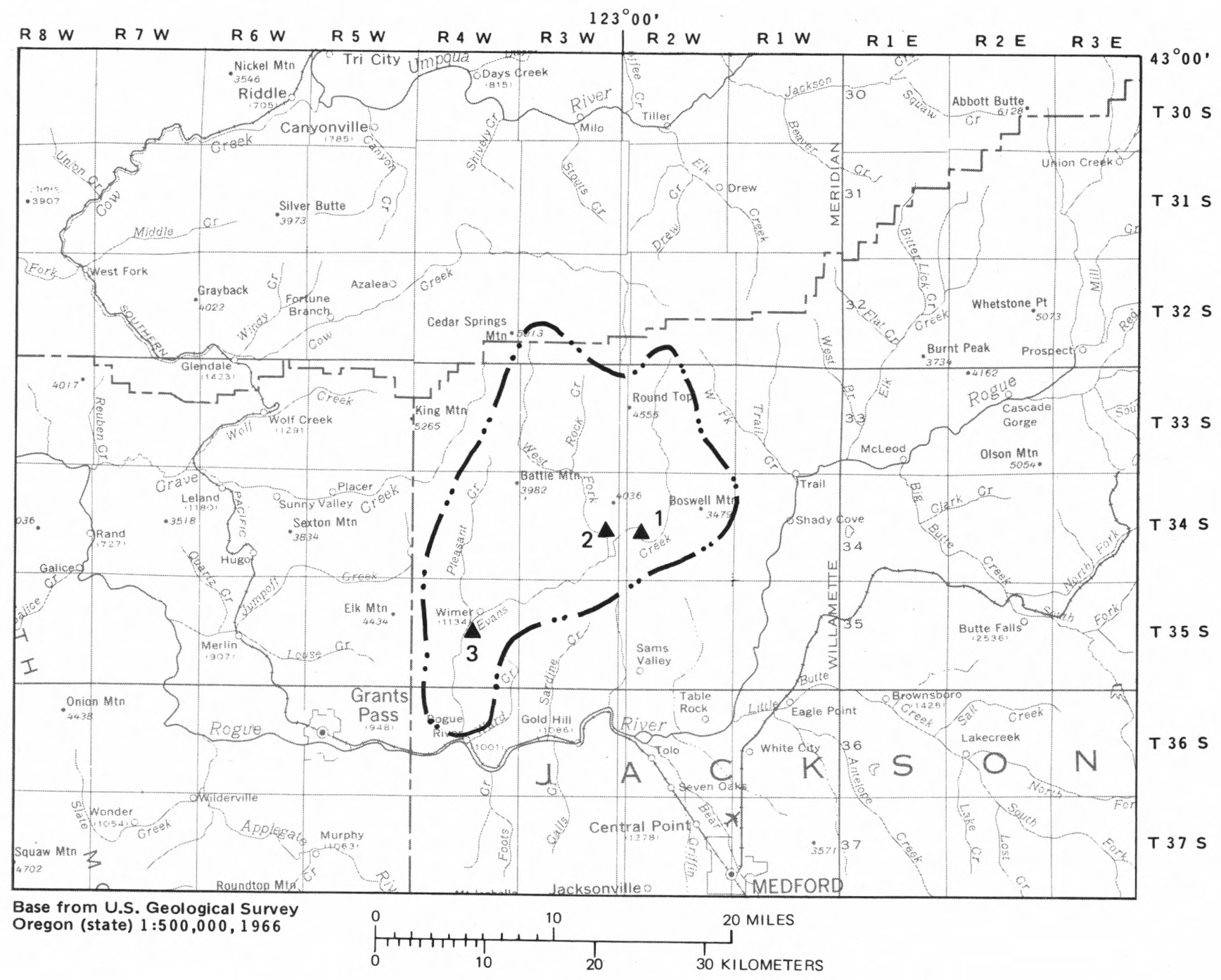

Figure 2.-Sampling sites in Evans Creek basin. 


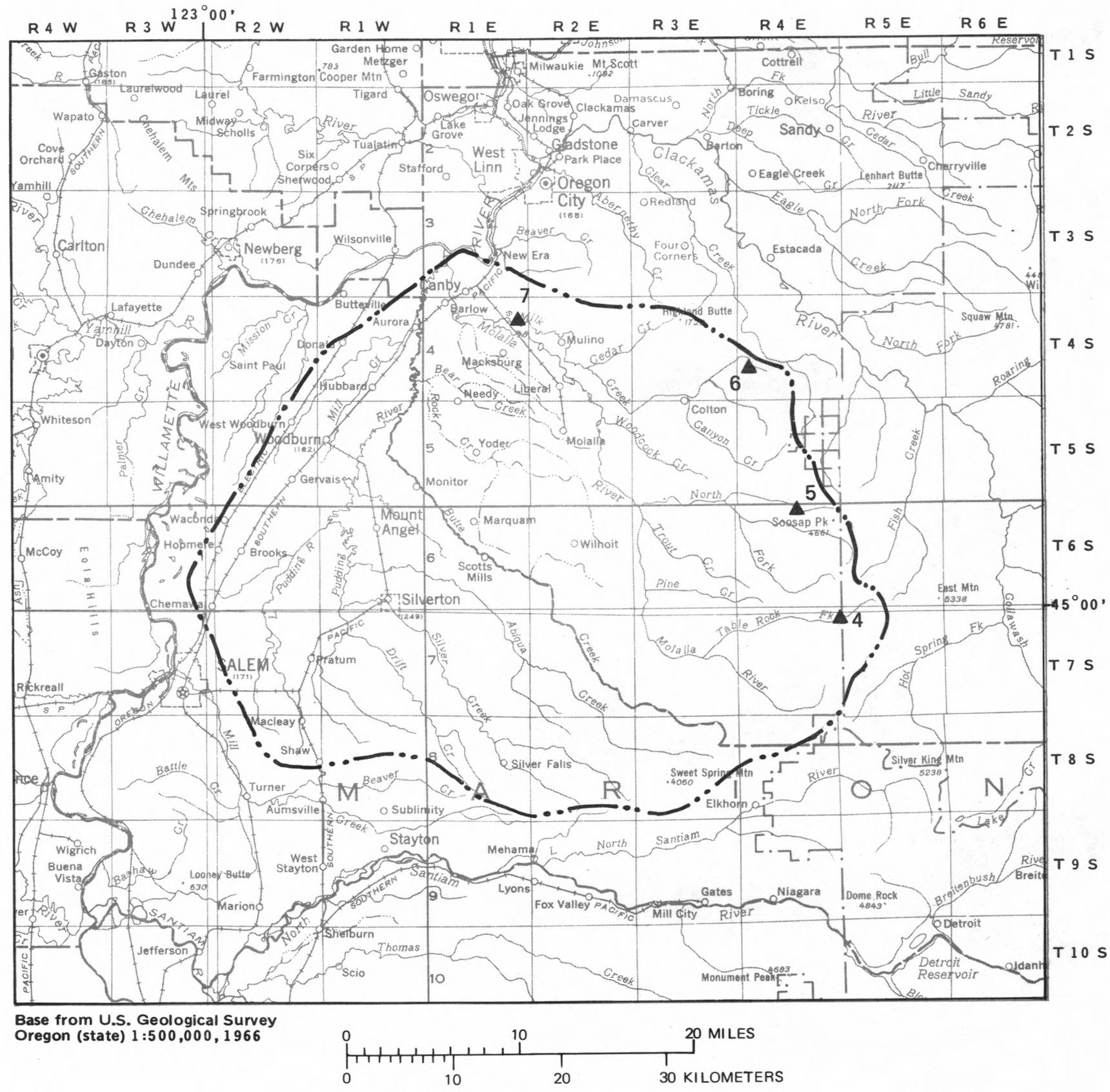

Figure 3.-Sampling sites in Molalla River basin. 


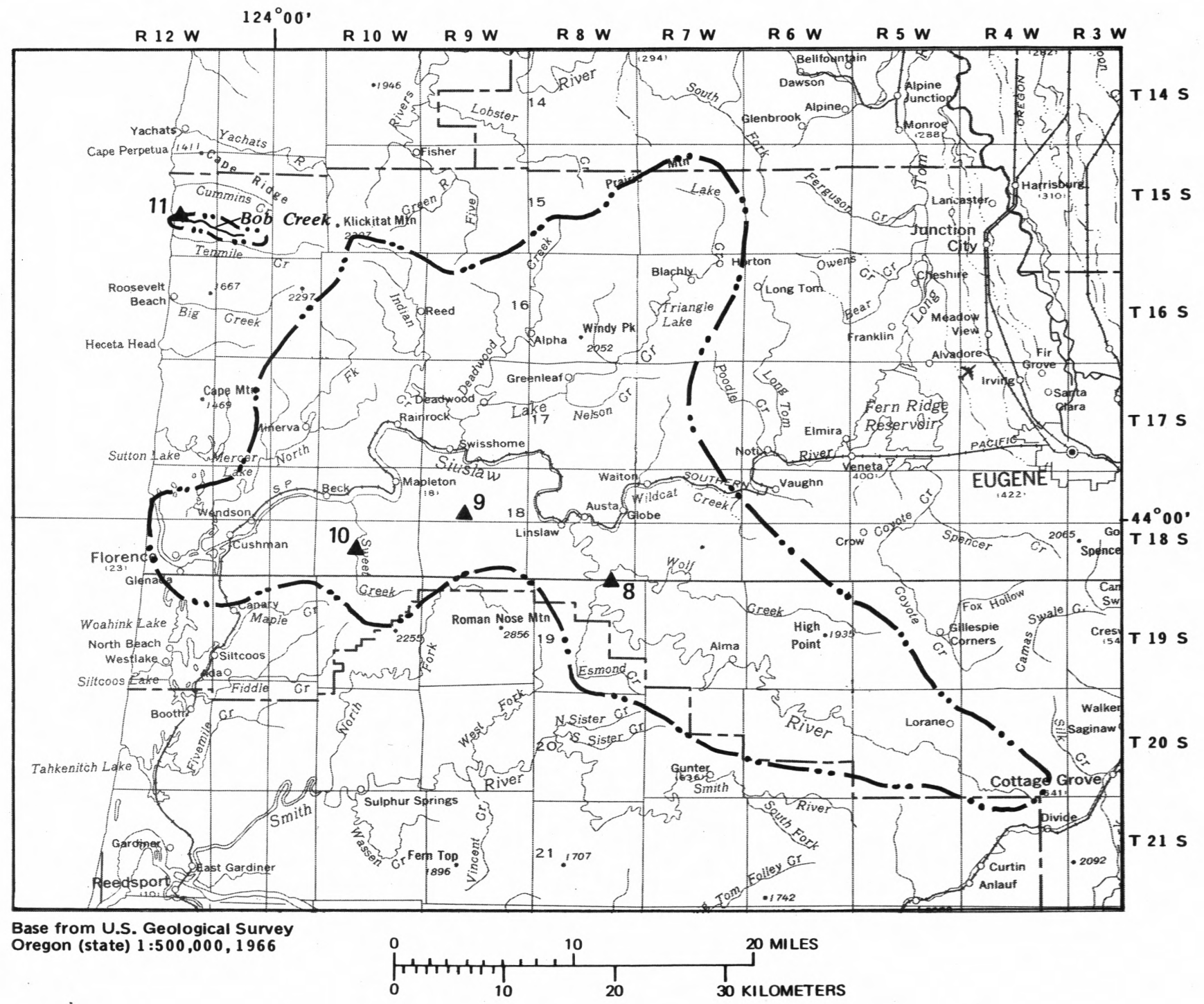

Figure 4.-Sampling sites in Siuslaw basin area . 


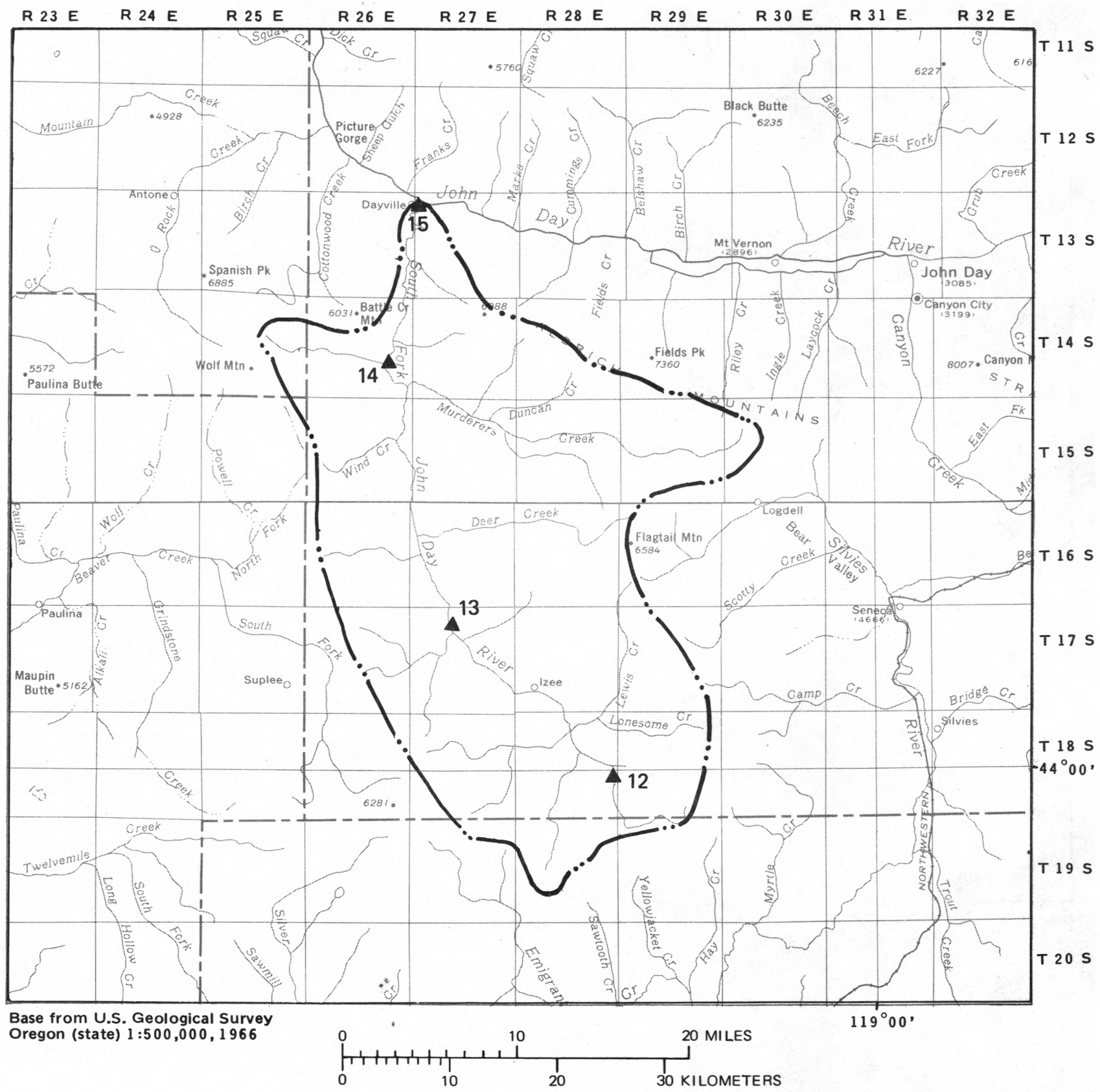

Figure 5.-Sampling sites in South Fork Day River basin. 
$119^{\circ} 00$ '

R26E R27E R28E R29E R 30 E R 31 E R 32 E R 33 E R 34 E R 35 E R 36 E R37E R38E

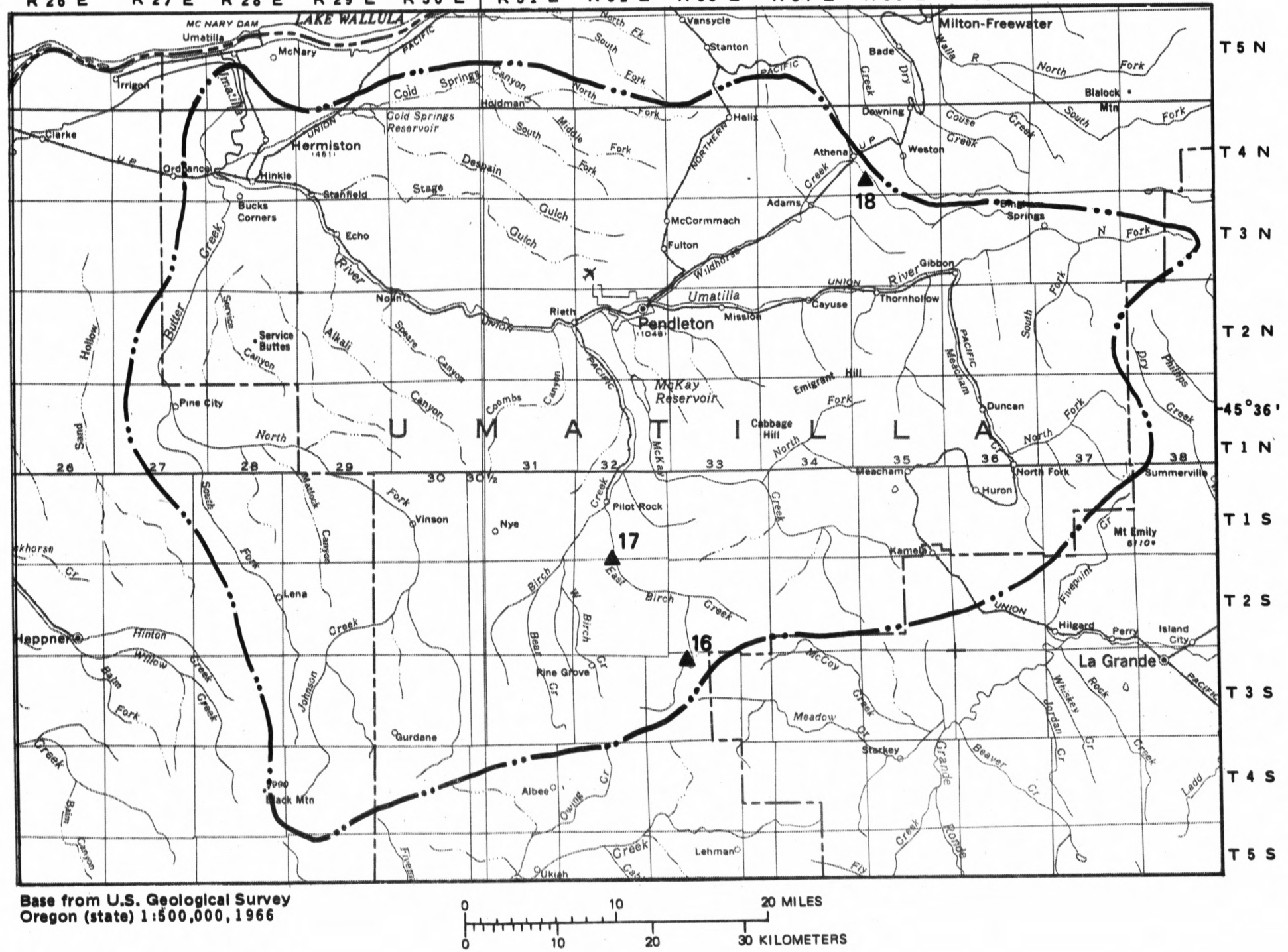

Figure 6.-Sampling sites in Umatilla River basin. 
Site number and name: 1A - Evans Creek above West Fork

Date: August 11-12, 1977

Discharge $\left(\mathrm{ft}^{3} / \mathrm{s}\right): 0.25$

\begin{tabular}{|c|c|c|c|c|c|c|}
\hline $\begin{array}{l}\text { Time } \\
(2400 \\
\text { hours) }\end{array}$ & $\begin{array}{l}\text { Water } \\
\text { tempera- } \\
\text { ture } \\
\left({ }^{\circ} \mathrm{C}\right)\end{array}$ & $\begin{array}{l}\text { Specific } \\
\text { conductance } \\
\text { (micromhos/ } \\
\mathrm{cm} \text { at } 25^{\circ} \mathrm{C} \text { ) }\end{array}$ & $\begin{array}{l}\text { Dissolved } \\
\text { oxygen } \\
\text { (mg/L) }\end{array}$ & $\begin{array}{l}\text { Dissolved } \\
\text { oxygen } \\
\text { (percent } \\
\text { saturation) }\end{array}$ & $\begin{array}{c}\mathrm{pH} \\
\text { (units) }\end{array}$ & $\begin{array}{l}\text { Pyrometer } \\
\text { (calories/ } \\
\mathrm{cm}^{2} / \mathrm{min} \text { ) }\end{array}$ \\
\hline \multicolumn{7}{|c|}{ August 11} \\
\hline 1400 & 23.0 & 162 & 9.0 & 111 & 8.4 & -- \\
\hline 1500 & 24.5 & 164 & 8.5 & 109 & 8.6 & -- \\
\hline 1600 & 26.1 & 165 & 8.1 & 106 & 8.7 & -- \\
\hline 1700 & 30.0 & .154 & 7.8 & 109 & 8.8 & -- \\
\hline 1800 & 28.6 & 159 & 7.5 & 104 & 8.5 & -- \\
\hline 1900 & 26.6 & 160 & 7.3 & 97 & 8.2 & -- \\
\hline 2000 & 25.2 & 164 & 7.3 & 94 & 8.0 & -- \\
\hline 2100 & 24.1 & 163 & 7.3 & 92 & 7.8 & -- \\
\hline 2200 & 23.1 & 165 & 7.3 & 90 & 7.7 & -- \\
\hline 2300 & 22.2 & 167 & 7.3 & 89 & 7.6 & -- \\
\hline 2400 & 21.4 & 167 & 7.4 & 89 & 7.5 & -- \\
\hline \multicolumn{7}{|c|}{ August 12} \\
\hline 0100 & 20.7 & 168 & 7.4 & 88 & 7.4 & -- \\
\hline 0200 & 20.2 & 170 & 7.5 & 88 & 7.4 & -- \\
\hline 0300 & 19.5 & 170 & 7.6 & 88 & 7.4 & -- \\
\hline 0400 & 19.0 & 170 & 7.6 & 87 & 7.4 & -- \\
\hline 0500 & 18.6 & 171 & 7.7 & 88 & 7.4 & -- \\
\hline 0600 & 18.3 & 169 & 7.8 & 89 & 7.4 & -- \\
\hline 0700 & 18.0 & 169 & 7.8 & 89 & 7.4 & -- \\
\hline 0800 & 17.9 & 168 & 7.9 & 89 & 7.5 & -- \\
\hline 0900 & 18.4 & 168 & 8.3 & 94 & 7.8 & -- \\
\hline 1000 & 19.3 & 168 & 8.6 & 100 & 7.9 & -- \\
\hline 1100 & 20.5 & 167 & 8.7 & 104 & 8.0 & -- \\
\hline 1200 & 22.0 & 165 & 8.8 & 107 & 8.2 & -- \\
\hline
\end{tabular}


Site number and name: 1B - Evans Creek above West Fork

Date: July 6-7, 1978

Discharge $\left(\mathrm{ft}^{3} / \mathrm{s}\right): 5.9$

\begin{tabular}{|c|c|c|c|c|c|c|}
\hline $\begin{array}{l}\text { Time } \\
(2400 \\
\text { hours })\end{array}$ & $\begin{array}{l}\text { Water } \\
\text { tempera- } \\
\text { ture } \\
\left({ }^{\circ} \mathrm{C}\right)\end{array}$ & $\begin{array}{l}\text { Specific } \\
\text { conductance } \\
\text { (micromhos/ } \\
\mathrm{cm} \text { at } 25^{\circ} \mathrm{C} \text { ) }\end{array}$ & $\begin{array}{l}\text { Dissolved } \\
\text { oxygen } \\
(\mathrm{mg} / \mathrm{L})\end{array}$ & $\begin{array}{l}\text { Dissolved } \\
\text { oxygen } \\
\text { (percent } \\
\text { saturation) }\end{array}$ & (units) & $\begin{array}{l}\text { Pyrometer } \\
\text { (calories/ } \\
\mathrm{cm}^{2} / \mathrm{min} \text { ) }\end{array}$ \\
\hline \multicolumn{7}{|l|}{ July 6} \\
\hline 1500 & 21.2 & 140 & 9.3 & 104 & 8.0 & -- \\
\hline 1600 & 21.7 & 145 & 8.8 & 99 & 7.9 & -- \\
\hline 1700 & 21.6 & 147 & 8.7 & 98 & 7.8 & -- \\
\hline 1800 & 21.3 & 148 & 8.6 & 96 & 7.7 & -- \\
\hline 1900 & 21.1 & 149 & 8.5 & 95 & 7.6 & -- \\
\hline 2000 & 21.1 & 148 & 8.3 & 92 & 7.5 & -- \\
\hline 2100 & 21.1 & 147 & 8.1 & 90 & 7.4 & -- \\
\hline 2200 & 20.8 & 145 & 8.0 & 89 & 7.3 & -- \\
\hline 2300 & 20.3 & 145 & 8.0 & 88 & 7.3 & -- \\
\hline 2400 & 19.8 & 145 & 8.1 & 88 & 7.3 & -- \\
\hline \multicolumn{7}{|l|}{ July 7} \\
\hline 0100 & 19.4 & 144 & 8.2 & 88 & 7.2 & -- \\
\hline 0200 & 19.0 & 144 & 8.2 & 88 & 7.1 & -- \\
\hline 0300 & 18.6 & 146 & 8.2 & 87 & 7.1 & -- \\
\hline 0400 & 18.4 & 147 & 8.2 & 87 & 7.2 & -- \\
\hline 0500 & 18.2 & 149 & 8.3 & 87 & 7.2 & -- \\
\hline 0600 & 17.9 & 149 & 8.4 & 88 & 7.2 & -- \\
\hline 0700 & 17.6 & 146 & 8.4 & 87 & 7.2 & -- \\
\hline 0800 & 17.3 & 139 & 8.5 & 88 & 7.0 & -- \\
\hline 0900 & 17.5 & 140 & 8.6 & 89 & 7.2 & -- \\
\hline 1000 & 17.9 & 141 & 8.9 & 93 & 7.6 & -- \\
\hline 1100 & 18.5 & 143 & 9.0 & 95 & 7.8 & -- \\
\hline 1200 & 19.3 & 143 & 9.2 & 99 & 8.0 & -- \\
\hline 1300 & 20.3 & 142 & 9.0 & 99 & 8.2 & -- \\
\hline 1400 & 21.4 & 143 & 9.0 & 101 & 8.3 & -- \\
\hline
\end{tabular}


Site number and name: 2 - West Fork Evans Creek

Date: August 11-12, 1977

Discharge $\left(\mathrm{ft}^{3} / \mathrm{s}\right): 3.4$

\begin{tabular}{|c|c|c|c|c|c|c|}
\hline $\begin{array}{l}\text { Time } \\
(2400 \\
\text { hours })\end{array}$ & $\begin{array}{l}\text { Water } \\
\text { tempera- } \\
\text { ture } \\
\left({ }^{\circ} \mathrm{C}\right)\end{array}$ & $\begin{array}{l}\text { Specific } \\
\text { conductance } \\
\text { (micromhos/ } \\
\mathrm{cm} \text { at } 25^{\circ} \mathrm{C} \text { ) }\end{array}$ & $\begin{array}{l}\text { Dissolved } \\
\text { oxygen } \\
\text { (mg/L) }\end{array}$ & $\begin{array}{l}\text { Dissolved } \\
\text { oxygen } \\
\text { (percent } \\
\text { saturation) }\end{array}$ & $\begin{array}{c}\mathrm{pH} \\
\text { (units) }\end{array}$ & $\begin{array}{c}\text { Pyrometer } \\
\text { (calories/ } \\
\mathrm{cm}^{2} / \mathrm{min} \text { ) }\end{array}$ \\
\hline
\end{tabular}

August 11

\begin{tabular}{|c|c|c|c|c|c|c|}
\hline 1200 & 23.2 & 254 & 9.2 & 114 & 8.2 & 1.2 \\
\hline 1300 & 24.8 & 252 & 9.2 & 116 & 8.5 & 1.2 \\
\hline 1400 & 26.0 & 250 & 9.2 & 119 & 8.7 & 1.2 \\
\hline 1500 & 27.0 & 249 & 9.2 & 121 & 8.8 & 1.2 \\
\hline 1600 & 27.2 & 247 & 9.2 & 123 & 8.9 & .80 \\
\hline 1700 & $27 . C$ & 246 & 8.6 & 113 & 8.8 & .40 \\
\hline 1800 & 26.6 & 245 & 8.0 & 105 & 8.7 & .18 \\
\hline 1900 & 26.1 & 245 & 7.4 & 96 & 8.5 & .08 \\
\hline 2000 & 25.6 & 245 & 7.0 & 90 & 8.2 & .04 \\
\hline 2100 & 25.0 & 245 & 6.6 & 84 & 7.8 & 0 \\
\hline 2200 & 24.4 & 245 & 6.2 & 78 & 7.7 & 0 \\
\hline 2300 & 23.8 & 245 & 6.0 & 74 & 7.6 & 0 \\
\hline 2400 & 23.2 & 246 & 5.9 & 73 & 7.5 & 0 \\
\hline Aug. & & & & & & \\
\hline 0100 & 22.6 & 248 & 5.8 & 71 & 7.5 & 0 \\
\hline 0200 & 22.0 & 251 & 5.8 & 70 & 7.5 & 0 \\
\hline 0300 & 21.4 & 254 & 6.1 & 73 & 7.5 & 0 \\
\hline 0400 & 20.9 & 258 & 6.4 & 75 & 7.5 & 0 \\
\hline 0500 & 20.4 & 262 & 6.8 & 79 & 7.5 & 0 \\
\hline 0600 & 19.8 & 265 & 7.3 & 85 & 7.5 & 0 \\
\hline 0700 & 19.4 & 266 & 7.8 & 90 & 7.6 & .04 \\
\hline 0800 & 19.4 & 266 & 8.1 & 93 & 7.6 & .10 \\
\hline 0900 & 19.9 & 264 & 8.5 & 99 & 7.7 & .16 \\
\hline 1000 & 20.8 & 262 & 8.9 & 105 & 7.9 & .40 \\
\hline 1100 & 22.0 & 260 & 9.3 & 112 & 8.0 & 1.0 \\
\hline 1200 & 23.2 & 258 & 9.6 & 118 & 8.1 & 1.1 \\
\hline
\end{tabular}


Site number and name: 2 - West Fork Evans Creek

Date: September 8-9, 1977

Discharge $\left(\mathrm{ft}^{3} / \mathrm{s}\right): 4.7$

\begin{tabular}{|c|c|c|c|c|c|c|}
\hline $\begin{array}{l}\text { Time } \\
(2400 \\
\text { hours })\end{array}$ & $\begin{array}{l}\text { Water } \\
\text { tempera- } \\
\text { ture } \\
\left({ }^{\circ} \mathrm{C}\right)\end{array}$ & $\begin{array}{l}\text { Specific } \\
\text { conductance } \\
\text { (micromhos/ } \\
\mathrm{cm} \text { at } 25^{\circ} \mathrm{C} \text { ) }\end{array}$ & $\begin{array}{l}\text { Dissolved } \\
\text { oxygen } \\
\text { (mg/L) }\end{array}$ & $\begin{array}{l}\text { Dissolved } \\
\text { oxygen } \\
\text { (percent } \\
\text { saturation) }\end{array}$ & $\begin{array}{c}\mathrm{pH} \\
\text { (units) }\end{array}$ & $\begin{array}{c}\text { Pyrometer } \\
\text { (calories/ } \\
\mathrm{cm}^{2} / \mathrm{min} \text { ) }\end{array}$ \\
\hline
\end{tabular}

\section{$\frac{\text { Sept. } 8}{1200}$}

1300

1400

1500

1600

1700

1800

1900

2000

2100

2200

2300

2400

Sept. 9

$\begin{array}{ll}0100 & 15.8 \\ 0200 & 15.5 \\ 0300 & 14.8 \\ 0400 & 14.4 \\ 0500 & 14.0 \\ 0600 & 13.6 \\ 0700 & 13.2 \\ 0800 & 13.0 \\ 0900 & 13.2 \\ 1000 & 14.4 \\ 1100 & 15.6\end{array}$

--
250
--
260

$--$

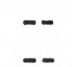

260

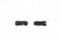

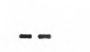

--

$-$

$--$

16.6

16.0

15.8

15.5

14.8

14.4

4.0

13.6

13.2

13.0

13.2

14.4

15.6

268

\section{6}

10.5

10.3

10.1

119

122

123

122

\section{9}

9.6

9.1

8.8

8.8

8.9

9.0

9.1

9.2

9.4

9.5

9.5

9.6

9.6

9.7

9.9

10.4

10.8

10.9

10.8

\section{8}

114

106

100

99

99

98

99

98

100

101

100

99

99

99

100

104

109

112

115

$\begin{array}{ll}8.4 & 1.0 \\ 8.4 & 1.2 \\ 8.4 & 1.2 \\ 8.3 & 1.0\end{array}$

8.3

8.3

8.3

8.2

8.0

7.9

7.8

7.8

7.8

7.8

7.8

7.8

7.8

7.8

.56

.38

.08

.04

0

0

0

0

0

7.8

7.8

7.8

8.0

8.2

8.3

0

0

0

0

0 
Site number and name: 2 - West Fork Evans Creek

Date: July 6-7, 1978

Discharge $\left(\mathrm{ft}^{3} / \mathrm{s}\right): 20$

\begin{tabular}{|c|c|c|c|c|c|c|}
\hline $\begin{array}{l}\text { Time } \\
(2400 \\
\text { hours })\end{array}$ & $\begin{array}{l}\text { Water } \\
\text { tempera- } \\
\text { ture } \\
\left({ }^{\circ} \mathrm{C}\right)\end{array}$ & $\begin{array}{l}\text { Specific } \\
\text { conductance } \\
\text { (micromhos/ } \\
\mathrm{cm} \text { at } 25^{\circ} \mathrm{C} \text { ) }\end{array}$ & $\begin{array}{l}\text { Dissolved } \\
\text { oxygen } \\
\text { (mg/L) }\end{array}$ & $\begin{array}{l}\text { Dissolved } \\
\text { oxygen } \\
\text { (percent } \\
\text { saturation) }\end{array}$ & $\begin{array}{c}\mathrm{pH} \\
\text { (units) }\end{array}$ & $\begin{array}{l}\text { Pyrometer } \\
\text { (calories/ } \\
\mathrm{cm}^{2} / \mathrm{min} \text { ) }\end{array}$ \\
\hline
\end{tabular}

$\frac{\text { July } 6}{1400}$

1500

1600

1700

1800

1900

2000

2100

2200

2300

2400

$\frac{\mathrm{July} 7}{0100}$

0200

0300

0400

0500

0600

0700

0800

0900

1000

1100

1200

1300

$20.5 \quad 167$

21.3

21.8

22.1

168

167

167

167

168

167

166

166

168

168

167

167

166

165

164

165

165

164

164

166

165

164

167

18.9

$\begin{array}{rr}10.3 & 113 \\ 9.2 & 103 \\ 8.9 & 100 \\ 8.8 & 100\end{array}$

8.6

8.5

8.3

8.2

8.2

8.2

8.2

8.2

8.2

8.2

8.2

8.1

8.1

8.2

8.4

8.7

9.0

9.2

9.3

9.3

113
103
100
100
97

92

91

90

89

88

99
101
8.3

1.3

8.2

8.2

8.2

1.1

.90

8.1

.66

7.9

7.8

7.7

.28

7.6

7.5

0

7.5

7.4

0

7.3

7.2

7.2

7.2

7.2

.02

.06

7.2

7.2

7.3

7.4

7.4

7.4

.18

.54

.88

1.1

1.2

1.3 
Site number and name: 3 - Evans Creek at Minthorne Road

Date: September 7-8, 1977

Discharge $\left(\mathrm{ft}^{3} / \mathrm{s}\right): 2.6$

\begin{tabular}{|c|c|c|c|c|c|c|}
\hline $\begin{array}{l}\text { Time } \\
(2400 \\
\text { hours })\end{array}$ & $\begin{array}{l}\text { Water } \\
\text { tempera- } \\
\text { ture } \\
\left({ }^{\circ} \mathrm{C}\right)\end{array}$ & $\begin{array}{l}\text { Specific } \\
\text { conductance } \\
\text { (micromhos/ } \\
\mathrm{cm} \text { at } 25^{\circ} \mathrm{C} \text { ) }\end{array}$ & $\begin{array}{l}\text { Dissolved } \\
\text { oxygen } \\
\text { (mg/L) }\end{array}$ & $\begin{array}{l}\text { Dissolved } \\
\text { oxygnn } \\
\text { (percent } \\
\text { saturation) }\end{array}$ & $\begin{array}{c}\mathrm{pH} \\
\text { (units) }\end{array}$ & $\begin{array}{c}\text { Pyrometer } \\
\text { (calories/ } \\
\mathrm{cm}^{2} / \mathrm{min} \text { ) }\end{array}$ \\
\hline \multicolumn{7}{|c|}{ Sept. 7} \\
\hline 1000 & 18.7 & 228 & 6.9 & 78 & 7.7 & 0.88 \\
\hline 1100 & 19.3 & 228 & 7.2 & 84 & 7.6 & 1.0 \\
\hline 1200 & 19.9 & 228 & 7.6 & 89 & 7.7 & 1.1 \\
\hline 1300 & 21.1 & 228 & 8.1 & 96 & 7.9 & 1.1 \\
\hline 1400 & 22.9 & 228 & 8.5 & 105 & 8.0 & .14 \\
\hline 1500 & 23.7 & 228 & 8.8 & 110 & 8.0 & .08 \\
\hline 1600 & 23.5 & 228 & 8.7 & 109 & 7.9 & .08 \\
\hline 1700 & 23.1 & 228 & 8.3 & 102 & 7.9 & .08 \\
\hline 1800 & 22.7 & 228 & 7.9 & 98 & 7.8 & .06 \\
\hline 1900 & 22.0 & 228 & 7.6 & 93 & 7.7 & .02 \\
\hline 2000 & 21.5 & 228 & 7.3 & 89 & 7.6 & 0 \\
\hline 2100 & 21.1 & 228 & 7.1 & 84 & 7.5 & 0 \\
\hline 2200 & 20.7 & 226 & 7.0 & 83 & 7.4 & 0 \\
\hline 2300 & 20.3 & 226 & 6.9 & 81 & 7.4 & 0 \\
\hline 2400 & 20.0 & 226 & 6.8 & 79 & 7.3 & 0 \\
\hline \multicolumn{7}{|l|}{ Sept. 8} \\
\hline 0100 & 19.5 & 226 & 6.6 & 77 & 7.2 & 0 \\
\hline 0200 & 19.1 & 226 & 6.4 & 74 & 7.2 & 0 \\
\hline 0300 & 18.5 & 226 & 6.4 & 73 & 7.1 & 0 \\
\hline 0400 & 18.1 & 226 & 6.4 & 73 & 7.1 & 0 \\
\hline 0500 & 17.9 & 226 & 6.4 & 72 & 7.0 & 0 \\
\hline 0600 & 17.5 & 224 & 6.6 & 73 & 7.0 & 0 \\
\hline 0700 & 17.3 & 222 & 6.8 & 76 & 7.0 & .06 \\
\hline 0800 & 17.3 & 220 & 7.0 & 78 & 7.1 & .28 \\
\hline 0900 & 17.5 & 218 & 8.5 & 94 & 7.1 & .68 \\
\hline
\end{tabular}


Site number and name: 3 - Evans Creek at Minthorne Road

Date: Ju1y 5-6, 1978

Discharge $\left(\mathrm{ft}^{3} / \mathrm{s}\right): 28$

\begin{tabular}{|c|c|c|c|c|c|c|}
\hline $\begin{array}{l}\text { Time } \\
(2400 \\
\text { hours) }\end{array}$ & $\begin{array}{l}\text { Water } \\
\text { tempera- } \\
\text { ture } \\
\left({ }^{\circ} \mathrm{C}\right)\end{array}$ & $\begin{array}{l}\text { Specific } \\
\text { conductance } \\
\text { (micromhos/ } \\
\mathrm{cm} \text { at } 25^{\circ} \mathrm{C} \text { ) }\end{array}$ & $\begin{array}{l}\text { Dissolved } \\
\text { oxygen } \\
\text { (mg/L) }\end{array}$ & $\begin{array}{l}\text { Dissolved } \\
\text { oxygen } \\
\text { (percent } \\
\text { saturation) }\end{array}$ & $\begin{array}{c}\mathrm{pH} \\
\text { (units) }\end{array}$ & $\begin{array}{c}\text { Pyrometer } \\
\text { (calories/ } \\
\mathrm{cm}^{2} / \mathrm{min} \text { ) }\end{array}$ \\
\hline \multicolumn{7}{|l|}{ July 5} \\
\hline 1300 & 20.8 & 146 & 10.7 & 118 & 7.9 & 1.3 \\
\hline 1400 & 22.3 & 147 & 10.6 & 121 & 8.3 & .52 \\
\hline 1500 & 23.6 & 149 & 10.5 & 122 & 8.5 & .28 \\
\hline 1600 & 24.6 & 150 & 10.4 & 123 & 8.5 & .12 \\
\hline 1700 & 24.8 & 150 & 10.2 & 121 & 8.5 & .18 \\
\hline 1800 & 24.9 & 150 & 9.9 & 118 & 8.6 & .08 \\
\hline 1900 & 24.7 & 152 & 9.6 & 114 & 8.6 & .08 \\
\hline 2000 & 24.1 & 152 & 9.2 & 108 & 8.5 & .04 \\
\hline 2100 & 23.3 & 153 & 8.8 & 102 & 8.4 & 0 \\
\hline 2200 & 22.3 & 154 & 8.4 & 96 & 8.3 & 0 \\
\hline 2300 & 21.3 & 158 & 8.2 & 92 & 7.6 & 0 \\
\hline 2400 & 20.6 & 159 & 8.0 & 88 & 7.1 & 0 \\
\hline \multicolumn{7}{|l|}{ July 6} \\
\hline 0100 & 20.1 & 160 & 8.0 & 87 & 7.0 & 0 \\
\hline 0200 & 19.5 & 160 & 8.0 & 86 & 6.9 & 0 \\
\hline 0300 & 18.9 & 159 & 8.0 & 85 & 6.9 & 0 \\
\hline 0400 & 18.5 & 159 & 8.1 & 86 & 7.0 & 0 \\
\hline 0500 & 18.1 & 161 & 8.0 & 84 & 7.0 & 0 \\
\hline 0600 & 17.9 & 158 & 8.0 & 84 & 7.0 & .02 \\
\hline 0700 & 17.7 & 160 & 8.2 & 85 & 7.1 & .16 \\
\hline 0800 & 17.6 & 161 & 8.3 & 86 & 7.3 & .50 \\
\hline 0900 & 17.7 & 162 & 8.5 & 89 & 7.4 & .76 \\
\hline 1000 & 18.2 & 163 & 8.9 & 94 & 7.6 & 1.0 \\
\hline 1100 & 19.1 & 162 & 9.4 & 101 & 7.8 & 1.1 \\
\hline 1200 & 20.3 & 160 & 9.8 & 107 & 8.0 & 1.2 \\
\hline
\end{tabular}


Site number and name: 4 - Lost Creek, Molalla River basin

Date: July 21-22, 1977

Discharge $\left(\mathrm{ft}^{3} / \mathrm{s}\right): 5.9$

\begin{tabular}{|c|c|c|c|c|c|c|}
\hline $\begin{array}{l}\text { Time } \\
(2400 \\
\text { hours })\end{array}$ & $\begin{array}{l}\text { Water } \\
\text { tempera- } \\
\text { ture } \\
\left({ }^{\circ} \mathrm{C}\right)\end{array}$ & $\begin{array}{l}\text { Specific } \\
\text { conductance } \\
\text { (micromhos/ } \\
\mathrm{cm} \text { at } 25^{\circ} \mathrm{C} \text { ) }\end{array}$ & $\begin{array}{l}\text { Dissolved } \\
\text { oxygen } \\
\text { (mg/L) }\end{array}$ & $\begin{array}{l}\text { Dissolved } \\
\text { oxygen } \\
\text { (percent } \\
\text { saturation) }\end{array}$ & $\begin{array}{c}\mathrm{pH} \\
\text { (units) }\end{array}$ & $\begin{array}{l}\text { Pyrometer } \\
\text { (calories/ } \\
\mathrm{cm}^{2} / \mathrm{min} \text { ) }\end{array}$ \\
\hline
\end{tabular}

Ju1y 21

\begin{tabular}{lllllll}
\hline 1200 & 14.4 & 60 & 9.0 & 87 & 7.4 & 1.3 \\
1300 & 15.6 & 60 & 8.9 & 90 & 7.5 & 1.3 \\
1400 & 16.8 & 60 & 8.7 & 90 & 7.5 & 1.2 \\
1500 & 17.6 & 59 & 8.5 & 89 & 7.6 & 1.2 \\
1600 & 18.2 & 60 & 8.5 & 89 & 7.6 & 1.0 \\
1700 & 18.2 & 60 & 8.4 & 88 & 7.6 & .82 \\
1800 & 17.6 & 60 & 8.6 & 91 & 7.5 & .35 \\
1900 & 16.4 & 61 & 8.7 & 92 & 7.4 & .06 \\
2000 & 15.9 & 60 & 8.8 & 89 & 7.3 & 0 \\
2100 & 15.0 & 62 & 8.9 & 87 & 7.2 & 0 \\
2200 & 14.4 & 62 & 9.0 & 87 & 7.2 & 0 \\
2300 & 14.0 & 62 & 9.1 & 88 & 7.2 & 0 \\
2400 & 13.6 & 61 & 9.2 & 88 & 7.2 & 0 \\
Ju1y 22 & & & & & & \\
0100 & 13.2 & 62 & 9.3 & 88 & 7.2 & 0 \\
0200 & 12.8 & 62 & 9.3 & 88 & 7.2 & 0 \\
0300 & 12.6 & 63 & 9.4 & 89 & 7.2 & 0 \\
0400 & 12.4 & 62 & 9.4 & 87 & 7.1 & 0 \\
0500 & 12.2 & 62 & 9.5 & 88 & 7.1 & 0 \\
0600 & 12.0 & 62 & 9.5 & & & \\
0700 & 12.0 & 62 & 9.5 & 88 & 7.0 & 0 \\
0800 & 12.0 & 62 & 9.4 & 88 & 7.0 & .04 \\
0900 & 12.4 & 62 & 9.4 & 87 & 7.0 & .06 \\
1000 & 13.0 & 62 & 9.3 & 88 & 7.0 & .10 \\
1100 & 14.0 & 62 & 9.2 & 88 & 7.1 & .10 \\
1200 & 15.2 & 62 & 9.0 & 88 & 7.1 & .12 \\
& & & & & & .18
\end{tabular}


Site number and name: 5 - Dead Horse Canyon Creek, Molalla River basin

Date: September 15-16, 1977

Discharge $\left(\mathrm{ft}^{3} / \mathrm{s}\right): 3.2$

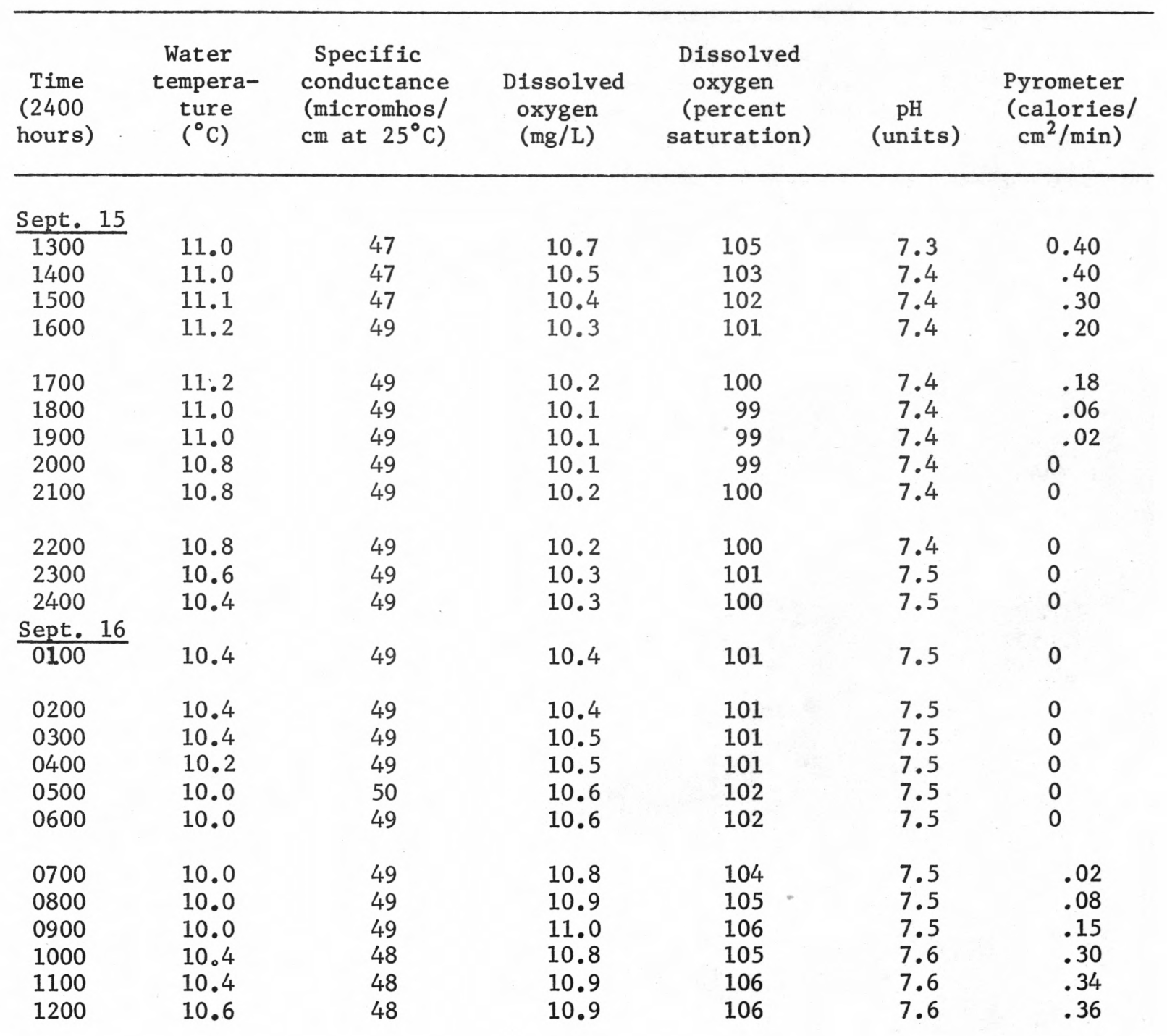


Site number and name: 6 - Dorn Creek, Molalla River basin

Date: September 14-15, 1977

Discharge $\left(\mathrm{ft}^{3} / \mathrm{s}\right): 0.14$

\begin{tabular}{|c|c|c|c|c|c|c|}
\hline $\begin{array}{l}\text { Time } \\
(2400 \\
\text { hours })\end{array}$ & $\begin{array}{l}\text { Water } \\
\text { tempera- } \\
\text { ture } \\
\left({ }^{\circ} \mathrm{C}\right)\end{array}$ & $\begin{array}{l}\text { Specific } \\
\text { conductance } \\
\text { (micromhos/ } \\
\mathrm{cm} \text { at } 25^{\circ} \mathrm{C} \text { ) }\end{array}$ & $\begin{array}{l}\text { Dissolved } \\
\text { oxygen } \\
\text { (mg/L) }\end{array}$ & $\begin{array}{l}\text { Dissolved } \\
\text { oxygen } \\
\text { (percent } \\
\text { saturation) }\end{array}$ & $\begin{array}{c}\mathrm{pH} \\
\text { (units) }\end{array}$ & $\begin{array}{c}\text { Pyrometer } \\
\text { (calories/ } \\
\mathrm{cm}^{2} / \mathrm{min} \text { ) }\end{array}$ \\
\hline \multicolumn{7}{|c|}{ Sept. 14} \\
\hline 1200 & 10.8 & 68 & 10.2 & 95 & 7.0 & 0.06 \\
\hline 1300 & 11.0 & 68 & 9.9 & 93 & 6.9 & .08 \\
\hline 1400 & 11.0 & 68 & 9.7 & 92 & 7.0 & .10 \\
\hline 1500 & 11.2 & 70 & 9.5 & 90 & 7.1 & .08 \\
\hline 1600 & 11.2 & 70 & 9.7 & 92 & 7.1 & .06 \\
\hline 1700 & 11.2 & 70 & 9.6 & 91 & 7.1 & .05 \\
\hline 1800 & 11.2 & 70 & 9.6 & 91 & 7.1 & .04 \\
\hline 1900 & 11.2 & 70 & 9.3 & 88 & 7.1 & 0 \\
\hline 2000 & 11.0 & 70 & 9.4 & 89 & 7.1 & 0 \\
\hline 2100 & -- & -- & -- & -- & -- & -- \\
\hline 2200 & -- & -- & -- & -- & -- & -- \\
\hline 2300 & -- & -- & -- & -- & -- & -- \\
\hline 2400 & -- & -- & -- & -- & -- & -- \\
\hline \multicolumn{7}{|c|}{ Sept. 15} \\
\hline 0100 & -- & -- & -- & -- & -- & -- \\
\hline 0200 & -- & -- & -- & -- & -- & -. \\
\hline 0300 & -- & -- & -- & -- & -- & -- \\
\hline 0400 & -- & -- & -- & -- & -- & -- \\
\hline 0500 & -- & -- & -- & -- & -- & -- \\
\hline 0600 & -- & -- & -- & -- & -- & -- \\
\hline 0700 & -- & -- & -- & -- & -- & -- \\
\hline 0800 & -- & -- & -- & -- & -- & -- \\
\hline 0900 & 10.0 & 70 & 10.6 & 98 & 7.1 & .10 \\
\hline 1000 & 10.2 & 70 & 10.4 & 96 & 7.1 & .10 \\
\hline
\end{tabular}


Site number and name: 7 - Milk Creek, Molalla River basin

Date: September 13-14, 1977

Discharge $\left(\mathrm{ft}^{3} / \mathrm{s}\right): 19$

\begin{tabular}{|c|c|c|c|c|c|c|}
\hline $\begin{array}{l}\text { Time } \\
(2400 \\
\text { hours })\end{array}$ & $\begin{array}{l}\text { Water } \\
\text { tempera- } \\
\text { ture } \\
\left({ }^{\circ} \mathrm{C}\right)\end{array}$ & $\begin{array}{l}\text { Specific } \\
\text { conductance } \\
\text { (micromhos/ } \\
\mathrm{cm} \text { at } 25^{\circ} \mathrm{C} \text { ) }\end{array}$ & $\begin{array}{l}\text { Dissolved } \\
\text { oxygen } \\
\text { (mg/L) }\end{array}$ & $\begin{array}{l}\text { Dissolved } \\
\text { oxygen } \\
\text { (percent } \\
\text { saturation) }\end{array}$ & $\underset{\text { (units) }}{\mathrm{pH}}$ & $\begin{array}{l}\text { Pyrometer } \\
\text { (calories/ } \\
\mathrm{cm}^{2} / \mathrm{min} \text { ) }\end{array}$ \\
\hline
\end{tabular}

Sept. 13

1100

1300

1400

1500

1600

1700

1800

1900

2000

2100

2200

2300

2400

Sept. 14

0200

0300

0400

$0500 \quad 17.1$

0600

0700

0800

0900

1000

\section{0 \\ 16.4 \\ 16.9 \\ 17.6}

$$
18.2
$$

18.8

19.4

19.5

19.5

$$
19.2
$$

18.8

18.8

18.8

18.4

18.4

18.2

17.8

17.6

17.1

16.9

16.6

16.4

16.2

16.0
88

88

88

88

88

88

88

88

88

88

88

88

88

88

88

88

88

88

88

88

88

88

88

88

\section{2}

10.2

10.2

10.3

10.4

10.5

10.7

10.6

10.3

9.9

9.4

9.2

8.9

8.8

\section{8}

8.9

8.8

8.8

9.0

9.0

9.2

9.4

9.5

9.7
102

103

104

107

109

112

115

114

111

106

100

98

95

93

93

93

92

92

93

92

94

95

96

97
7.7

7.8

7.8

7.8

7.9

7.9

7.9

7.9

7.8

7.7

7.5

7.5

7.4

7.3

7.3

7.3

7.3

7.3

7.2

7.2

7.2

7.2

7.2

7.3
0.86

.98

1.0

1.0

.92

.76

.60

.28

.10

0

0

0

0

0

0

0

0

0

0

0

0

.02

.08 
Site number and name: 8 - Wolf Creek, Siuslaw River basin

Date: October 4-5, 1977

Discharge $\left(\mathrm{ft}^{3} / \mathrm{s}\right): 8.5$

\begin{tabular}{|c|c|c|c|c|c|c|}
\hline $\begin{array}{l}\text { Time } \\
(2400 \\
\text { hours })\end{array}$ & $\begin{array}{l}\text { Water } \\
\text { tempera- } \\
\text { ture } \\
\left({ }^{\circ} \mathrm{C}\right)\end{array}$ & $\begin{array}{l}\text { Specific } \\
\text { conductance } \\
\text { (micromhos/ } \\
\mathrm{cm} \text { at } 25^{\circ} \mathrm{C} \text { ) }\end{array}$ & $\begin{array}{l}\text { Dissolved } \\
\text { oxygen } \\
(\mathrm{mg} / \mathrm{L})\end{array}$ & $\begin{array}{l}\text { Dissolved } \\
\text { oxygen } \\
\text { (percent } \\
\text { saturation) }\end{array}$ & $\begin{array}{c}\mathrm{pH} \\
\text { (units) }\end{array}$ & $\begin{array}{l}\text { Pyrometer } \\
\text { (calories/ } \\
\mathrm{cm}^{2} / \mathrm{min} \text { ) }\end{array}$ \\
\hline \multicolumn{7}{|l|}{ oct. 4} \\
\hline 1000 & 10.5 & 92 & 12.4 & 111 & 7.6 & -- \\
\hline 1100 & 10.7 & 92 & 12.2 & 109 & 7.6 & -- \\
\hline 1200 & 11.2 & 90 & 11.9 & 108 & 7.6 & -- \\
\hline 1300 & 11.9 & 84 & 11.7 & 108 & 7.6 & -- \\
\hline 1400 & 12.1 & 79 & 11.5 & 106 & 7.6 & -- \\
\hline 1500 & 12.0 & 73 & 11.3 & 104 & 7.5 & -- \\
\hline 1600 & 11.8 & 69 & 11.2 & 103 & 7.4 & -- \\
\hline 1700 & 11.3 & 66 & 11.0 & 100 & 7.3 & -- \\
\hline 1800 & 11.1 & 66 & 10.9 & 99 & 7.2 & -- \\
\hline 1900 & 10.8 & 68 & 10.9 & 98 & 7.2 & -- \\
\hline 2000 & 10.8 & 70 & 10.8 & 97 & 7.2 & -- \\
\hline 2100 & 11.1 & 72 & 10.8 & 98 & 7.2 & -- \\
\hline 2200 & 11.3 & 74 & 10.7 & 97 & 7.2 & -- \\
\hline 2300 & 11.4 & 74 & 10.7 & 97 & 7.2 & -- \\
\hline 2400 & 11.6 & 74 & 10.7 & 98 & 7.2 & -- \\
\hline \multicolumn{7}{|l|}{ oct. 5} \\
\hline 0100 & 11.7 & 74 & 10.6 & 97 & 7.2 & -- \\
\hline 0200 & 11.7 & 74 & 10.6 & 97 & 7.2 & -- \\
\hline 0300 & 11.7 & 74 & 10.5 & 96 & 7.2 & -- \\
\hline 0400 & 11.7 & 74 & 10.4 & 95 & 7.1 & -- \\
\hline 0500 & 11.5 & 73 & 10.4 & 95 & 7.0 & -- \\
\hline 0600 & 11.5 & 73 & 10.4 & 95 & 6.9 & -- \\
\hline 0700 & 11.4 & 72 & 10.3 & 94 & 6.9 & -- \\
\hline 0800 & 11.4 & 72 & 10.4 & 95 & 6.9 & -- \\
\hline 0900 & 11.3 & 71 & 10.5 & 95 & 6.9 & -- \\
\hline 1000 & 11.1 & 70 & 10.6 & 96 & 6.9 & -- \\
\hline
\end{tabular}


Site number and name: 9 - Knowles Creek, Siuslaw River basin

Date: October 4-5, 1977

Discharge $\left(\mathrm{ft}^{3} / \mathrm{s}\right): 2.7$

\begin{tabular}{|c|c|c|c|c|c|c|}
\hline $\begin{array}{l}\text { Time } \\
(2400 \\
\text { hours })\end{array}$ & $\begin{array}{l}\text { Water } \\
\text { tempera- } \\
\text { ture } \\
\left({ }^{\circ} \mathrm{C}\right)\end{array}$ & $\begin{array}{l}\text { Specific } \\
\text { conductance } \\
\text { (micromhos/ } \\
\mathrm{cm} \text { at } 25^{\circ} \mathrm{C} \text { ) }\end{array}$ & $\begin{array}{c}\text { Dissolved } \\
\text { oxygen } \\
(\mathrm{mg} / \mathrm{L})\end{array}$ & $\begin{array}{l}\text { Dissolved } \\
\text { oxygen } \\
\text { (percent } \\
\text { saturation) }\end{array}$ & $\begin{array}{c}\mathrm{pH} \\
\text { (units) }\end{array}$ & $\begin{array}{c}\text { Pyrometer } \\
\text { (calories/ } \\
\mathrm{cm}^{2} / \mathrm{min} \text { ) }\end{array}$ \\
\hline
\end{tabular}

\section{$\frac{\text { Oct. } 4}{1100}$}

1200

1300

1400

1500

1600

1700

1800

1900

2000

2100

2200

2300

2400

$\frac{\text { 0ct. } 5}{0100}$

0200

0300

0400

0500

0600

0700

0800

0900

1000

\section{4 \\ 9.8 \\ 10.2 \\ 10.2}

$$
10.4
$$

$$
10.2
$$$$
10.0
$$$$
10.0
$$$$
10.0
$$

\section{5}

65

65

65

64

63

63

63

63

63

62

62

62

62

62

62

62

62

62

61

61

61

61

61

\section{7}

12.0

11.9

11.6

11.3

11.2

11.0

10.9

10.9

10.9

10.9

10.9

10.9

10.9

11.0

11.0

11.0

11.1

11.2

11.2

11.3

11.3

11.4

1.1 .4
102

105

106

105

101

99

97

96

96

96

96

96

96

96

97

96

96

97

98

97

98

98

100

100
6.9

7.3

7.3

7.3

0.08

.08

.08

.10

7. 3

.10

7.2

.10

7.2

7.2

.08

.06

7.2

0

$\begin{array}{ll}7.2 & 0 \\ 7.2 & 0 \\ 7.2 & 0 \\ 7.2 & 0 \\ 7.3 & 0\end{array}$

7.3

7.3

7.3

7.3

7.3

7.3

7.3

7.3

7.3

7.3

$$
\begin{aligned}
& 0 \\
& 0 \\
& 0 \\
& 0
\end{aligned}
$$

0
0
0
.02
.08
.10 
Site number and name: 10 - Cedar Creek, Siuslaw River basin

Date: August 8-10, 1977

Discharge $\left(\mathrm{ft}^{3} / \mathrm{s}\right): 0.43$

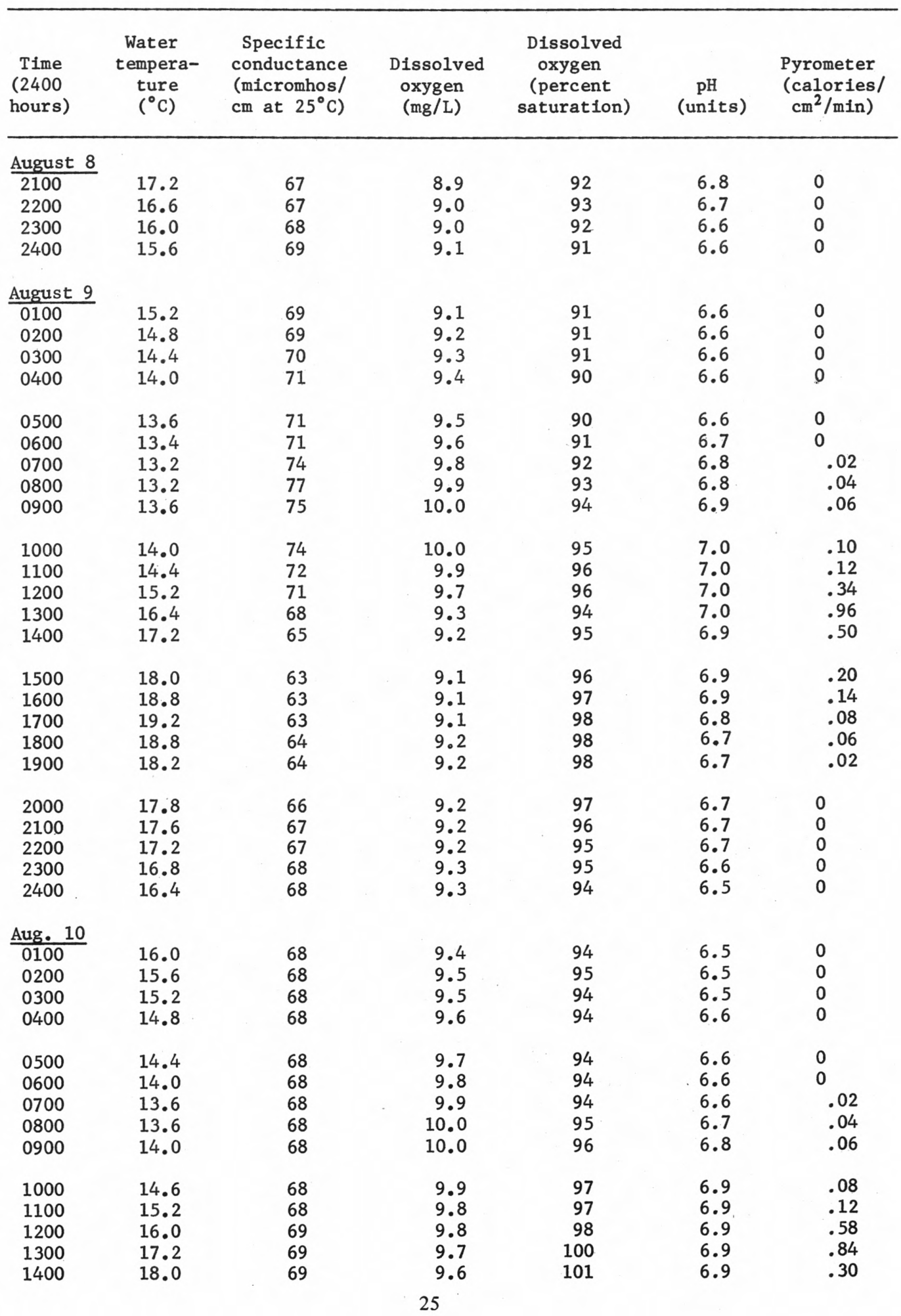


Table 2.--Physical characteristics--Continued

Site number and name: 10 - Cedar Creek, Siuslaw River basin

Date: October 5-6, 1977

Discharge $\left(\mathrm{ft}^{3} / \mathrm{s}\right): 2.4$

Remark: Recorder did not print during night.

\begin{tabular}{|c|c|c|c|c|c|c|}
\hline $\begin{array}{l}\text { Time } \\
(2400 \\
\text { hours })\end{array}$ & $\begin{array}{l}\text { Water } \\
\text { tempera- } \\
\text { ture } \\
\left({ }^{\circ} \mathrm{C}\right)\end{array}$ & $\begin{array}{l}\text { Specific } \\
\text { conductance } \\
\text { (micromhos/ } \\
\mathrm{cm} \text { at } 25^{\circ} \mathrm{C} \text { ) }\end{array}$ & $\begin{array}{l}\text { Dissolved } \\
\text { oxygen } \\
\text { (mg/L) }\end{array}$ & $\begin{array}{l}\text { Dissolved } \\
\text { oxygen } \\
\text { (percent } \\
\text { saturation) }\end{array}$ & $\begin{array}{c}\mathrm{pH} \\
\text { (units) }\end{array}$ & $\begin{array}{c}\text { Pyrometer } \\
\text { (calories/ } \\
\mathrm{cm}^{2} / \mathrm{min} \text { ) }\end{array}$ \\
\hline \multicolumn{7}{|l|}{ Oct. 5} \\
\hline 1330 & 10.9 & 58 & 12.2 & 110 & 7.1 & -- \\
\hline 1430 & 11.2 & 58 & 11.5 & 105 & 7.1 & -- \\
\hline 1530 & 11.4 & 57 & 11.2 & 102 & 7.2 & -- \\
\hline \multicolumn{7}{|l|}{ oct. 6} \\
\hline 1030 & 10.9 & 56 & 11.0 & 99 & 7.1 & -- \\
\hline 1130 & 11.0 & 56 & 10.6 & 95 & 7.2 & -- \\
\hline 1230 & 11.1 & 56 & 9.8 & 88 & 7.2 & -- \\
\hline
\end{tabular}


Site number and name: 11- Bob Creek, Siuslaw Basin

Date: August 8-9, 1977

Discharge $\left(\mathrm{ft}^{3} / \mathrm{s}\right): 2.7$

\begin{tabular}{|c|c|c|c|c|c|c|}
\hline $\begin{array}{l}\text { Time } \\
(2400 \\
\text { hours) }\end{array}$ & $\begin{array}{l}\text { Water } \\
\text { tempera- } \\
\text { ture } \\
\left({ }^{\circ} \mathrm{C}\right)\end{array}$ & $\begin{array}{l}\text { Specific } \\
\text { conductance } \\
\text { (micromhos/ } \\
\mathrm{cm} \text { at } 25^{\circ} \mathrm{C} \text { ) }\end{array}$ & $\begin{array}{l}\text { Dissolved } \\
\text { oxygen } \\
\text { (mg/L) }\end{array}$ & $\begin{array}{l}\text { Dissolved } \\
\text { oxygen } \\
\text { (percent } \\
\text { saturation) }\end{array}$ & $\begin{array}{c}\mathrm{pH} \\
\text { (units) }\end{array}$ & $\begin{array}{l}\text { Pyrometer } \\
\text { (calories/ } \\
\mathrm{cm}^{2} / \mathrm{min} \text { ) }\end{array}$ \\
\hline
\end{tabular}

August 8

\begin{tabular}{|c|c|c|c|c|c|c|}
\hline 1900 & 16.6 & 79 & 9.4 & 96 & 6.6 & -- \\
\hline 2000 & 16.3 & 77 & 9.0 & 91 & 6.6 & -- \\
\hline 2100 & 16.0 & 75 & 8.6 & 86 & 6.4 & -- \\
\hline 2200 & 15.6 & 74 & 8.6 & 86 & 6.3 & -- \\
\hline 2300 & 15.3 & 73 & 8.8 & 87 & 6.3 & -- \\
\hline 2400 & 15.1 & 74 & 8.9 & 88 & 6.4 & -- \\
\hline \multicolumn{7}{|l|}{ August 9} \\
\hline 0100 & 14.8 & 75 & 9.0 & 88 & 6.5 & -- \\
\hline 0200 & 14.7 & 78 & 9.2 & 90 & 6.6 & -- \\
\hline 0300 & 14.6 & 80 & 9.3 & 91 & 6.7 & -- \\
\hline 0400 & 14.5 & 81 & 9.4 & 91 & 6.8 & -- \\
\hline 0500 & 14.3 & 82 & 9.6 & 93 & 6.9 & -- \\
\hline 0600 & 13.9 & 82 & 9.6 & 92 & 6.9 & -- \\
\hline 0700 & 13.7 & 81 & 9.6 & 92 & 6.9 & -- \\
\hline 0800 & 13.6 & 77 & 9.8 & 93 & 6.9 & -- \\
\hline 0900 & 13.5 & 73 & 10.0 & 95 & 6.9 & -- \\
\hline 1000 & 13.9 & 73 & 10.1 & 97 & 6.9 & -- \\
\hline 1100 & 14.4 & 73 & 10.1 & 98 & 6.9 & -- \\
\hline 1200 & 15.0 & 73 & 10.0 & 98 & 6.9 & -- \\
\hline 1300 & 15.7 & 73 & 9.9 & 99 & 6.8 & -- \\
\hline 1400 & 16.4 & 73 & 9.8 & 100 & 6.8 & -- \\
\hline 1500 & 17.0 & 73 & 9.6 & 99 & 6.8 & -- \\
\hline 1600 & 17.0 & 73 & 9.5 & 98 & 6.7 & -- \\
\hline 1700 & 17.0 & 73 & 9.4 & 97 & 6.7 & -- \\
\hline 1800 & 17.0 & 74 & 9.3 & 96 & 6.7 & \\
\hline
\end{tabular}


Site number and name: 11 - Bob Creek, Siuslaw Basin

Date: October 5-6, 1977

Discharge $\left(\mathrm{ft}^{3} / \mathrm{s}\right): 9.2$

\begin{tabular}{|c|c|c|c|c|c|c|}
\hline $\begin{array}{l}\text { Time } \\
(2400 \\
\text { hours })\end{array}$ & $\begin{array}{l}\text { Water } \\
\text { tempera- } \\
\text { ture } \\
\left({ }^{\circ} \mathrm{C}\right)\end{array}$ & $\begin{array}{l}\text { Specific } \\
\text { conductance } \\
\text { (micromhos/ } \\
\mathrm{cm} \text { at } 25^{\circ} \mathrm{C} \text { ) }\end{array}$ & $\begin{array}{l}\text { Dissolved } \\
\text { oxygen } \\
(\mathrm{mg} / \mathrm{L})\end{array}$ & $\begin{array}{c}\text { Dissolved } \\
\text { oxygen } \\
\text { (percent } \\
\text { saturation) }\end{array}$ & $\begin{array}{c}\mathrm{pH} \\
\text { (units) }\end{array}$ & $\begin{array}{c}\text { Pyrometer } \\
\text { (calories/ } \\
\mathrm{cm}^{2} / \mathrm{min} \text { ) }\end{array}$ \\
\hline \multicolumn{7}{|l|}{ Oct. 5} \\
\hline 1800 & 11.2 & 58 & 11.0 & 100 & 7.2 & 0.12 \\
\hline 1900 & 11.0 & 58 & 11.0 & 100 & 7.2 & 0 \\
\hline 2000 & 10.8 & 58 & 11.0 & 99 & 7.1 & 0 \\
\hline 2100 & 10.8 & 59 & 10.9 & 98 & 7.1 & 0 \\
\hline 2200 & 10.6 & 59 & 10.9 & 97 & 7.1 & 0 \\
\hline 2300 & 10.6 & 59 & 10.9 & 97 & 7.0 & 0 \\
\hline 2400 & 10.4 & 59 & 10.9 & 97 & 7.0 & 0 \\
\hline \multicolumn{7}{|l|}{ Oct. 6} \\
\hline 0100 & 10.4 & 59 & 10.9 & 97 & 7.0 & 0 \\
\hline 0200 & 10.4 & 59 & 10.9 & 97 & 7.0 & 0 \\
\hline 0300 & 10.4 & 59 & 10.9 & 97 & 7.0 & 0 \\
\hline 0400 & 10.4 & 59 & 11.0 & 98 & 7.0 & 0 \\
\hline 0500 & 10.4 & 59 & 11.0 & 98 & 7.0 & 0 \\
\hline 0600 & 10.4 & 59 & 11.1 & 99 & 7.0 & 0 \\
\hline 0700 & 10.4 & 59 & 11.1 & 99 & 7.0 & 0 \\
\hline 0800 & 10.6 & 59 & 11.2 & 100 & 7.0 & 0 \\
\hline 0900 & 10.6 & 59 & 11.2 & 100 & 7.0 & .02 \\
\hline 1000 & 10.6 & 59 & 11.2 & 100 & 7.0 & .04 \\
\hline 1100 & 10.8 & 59 & 11.2 & 101 & 7.0 & .08 \\
\hline 1200 & 10.8 & 59 & 11.2 & 101 & 7.0 & .12 \\
\hline 1300 & 10.8 & 59 & 11.2 & 101 & 7.0 & .12 \\
\hline 1400 & 11.0 & 60 & 11.1 & 101 & 7.0 & .12 \\
\hline 1500 & 11.0 & 60 & 11.1 & 101 & 7.0 & .18 \\
\hline 1600 & 11.2 & 60 & 11.1 & 101 & 7.0 & .50 \\
\hline 1700 & 11.2 & 60 & 11.1 & 101 & 7.0 & .40 \\
\hline 1800 & 11.2 & 60 & 11.1 & 101 & 7.0 & .08 \\
\hline
\end{tabular}


Site number and name: 12 - South Fork John Day River above Forest Service boundary

Date: September 14-15, 1978

Discharge $\left(\mathrm{ft}^{3} / \mathrm{s}\right): 0.5$

\begin{tabular}{|c|c|c|c|c|c|c|}
\hline $\begin{array}{l}\text { Time } \\
(2400 \\
\text { hours })\end{array}$ & $\begin{array}{l}\text { Water } \\
\text { tempera- } \\
\text { ture } \\
\left({ }^{\circ} \mathrm{C}\right)\end{array}$ & $\begin{array}{l}\text { Specific } \\
\text { conductance } \\
\text { (micromhos/ } \\
\mathrm{cm} \text { at } 25^{\circ} \mathrm{C} \text { ) }\end{array}$ & $\begin{array}{l}\text { Dissolved } \\
\text { oxygen } \\
\text { (mg/L) }\end{array}$ & $\begin{array}{l}\text { Dissolved } \\
\text { oxygen } \\
\text { (percent } \\
\text { saturation) }\end{array}$ & $\begin{array}{c}\mathrm{pH} \\
\text { (units) }\end{array}$ & $\begin{array}{c}\text { Pyrometer } \\
\text { (calories/ } \\
\mathrm{cm}^{2} / \mathrm{min} \text { ) }\end{array}$ \\
\hline
\end{tabular}

$\frac{\text { Sept. } 14}{1900}$

$\begin{array}{lll}13.0 & 338\end{array}$

$2000 \quad 12.2 \quad 340$

2100

2200

2300

2400

Sept. 15

0100

0200

0300

0400

0500

0600

0700

0800

0900

1000

1100

1200

1300

1400
11.4

10.6

340

339

342

341

343

344

344

344

345

343

345

346

350

352

358

364

343

326
9.2

8.9

8.9

9.3

9.5

9.6

9.9

10.1

10.2

10.4

10.5

10.6

10.7

10.9

11.4

11.4

10.9

10.9

10.7

10.2
87

83

81

83

84

84

86

87

86

88

88

88

89

90

96

98

97

100

101

101
8.

8.1

8.1

8.1

8.2

8.2

8.3

8.3

8.3

8.2

8.2

8.2

8.2

8.2

8.3

8.3

8.2

8.3

8.3

8.4
$-$

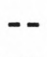

$-$ 
Table 2.--Physical characteristics--Continued

Site number and name: 13 - South Fork John Day River above Little Pine Creek

Date: September 15, 1978

Discharge $\left(\mathrm{ft}^{3} / \mathrm{s}\right): 16$

\begin{tabular}{|c|c|c|c|c|c|c|}
\hline $\begin{array}{l}\text { Time } \\
(2400 \\
\text { hours) }\end{array}$ & $\begin{array}{l}\text { Water } \\
\text { tempera- } \\
\text { ture } \\
\left({ }^{\circ} \mathrm{C}\right)\end{array}$ & $\begin{array}{l}\text { Specific } \\
\text { conductance } \\
\text { (micromhos/ } \\
\mathrm{cm} \text { at } 25^{\circ} \mathrm{C} \text { ) }\end{array}$ & $\begin{array}{c}\text { Dissolved } \\
\text { oxygen } \\
(\mathrm{mg} / \mathrm{L})\end{array}$ & $\begin{array}{l}\text { Dissolved } \\
\text { oxygen } \\
\text { (percent } \\
\text { saturation) }\end{array}$ & $\begin{array}{c}\mathrm{pH} \\
\text { (units) }\end{array}$ & $\begin{array}{l}\text { Pyrometer } \\
\text { (calories/ } \\
\mathrm{cm}^{2} / \mathrm{min} \text { ) }\end{array}$ \\
\hline
\end{tabular}

$\frac{\text { Sept. } 15}{1000}$

1100

10.0

460

468

1200

10.8

11.3

11.9

472

1300

480

10.4

10.5

10.7

10.7

8.2

0.70

1400

12.5

485

1500

13.5

1600

13.7

1700

13.7

1800

13.8

494

494

490

490

$\begin{array}{rr}10.5 & 98 \\ 10.3 & 98 \\ 10.0 & 96 \\ 9.6 & 92 \\ 9.3 & 89\end{array}$

8.3

.80

97

8.4

.88

99

.78

98

8.4

$\begin{array}{ll}8.4 & .90 \\ 8.4 & .82\end{array}$

8.3

.56

8.3

.25

89

8.2

.08 
Site number and name: 14 - Black Canyon Creek, South Fork John Day River basin

Date: September 13-14, 1978

Discharge $\left(\mathrm{ft}^{3} / \mathrm{s}\right): 9.1$

\begin{tabular}{|c|c|c|c|c|c|c|}
\hline $\begin{array}{l}\text { Time } \\
(2400 \\
\text { hours })\end{array}$ & $\begin{array}{l}\text { Water } \\
\text { tempera- } \\
\text { ture } \\
\left({ }^{\circ} \mathrm{C}\right)\end{array}$ & $\begin{array}{l}\text { Specific } \\
\text { conductance } \\
\text { (micromhos/ } \\
\mathrm{cm} \text { at } 25^{\circ} \mathrm{C} \text { ) }\end{array}$ & $\begin{array}{l}\text { Dissolved } \\
\text { oxygen } \\
\text { (mg/L) }\end{array}$ & $\begin{array}{l}\text { Dissolved } \\
\text { oxygen } \\
\text { (percent } \\
\text { saturation) }\end{array}$ & $\begin{array}{c}\mathrm{pH} \\
\text { (units) }\end{array}$ & $\begin{array}{l}\text { Pyrometer } \\
\text { (calories/ } \\
\mathrm{cm}^{2} / \mathrm{min} \text { ) }\end{array}$ \\
\hline
\end{tabular}

\section{$\frac{\text { Sept. } 13}{1400}$}

1500

1600

1700

1800

1900

2000

2100

2200

2300

2400

Sept. 14

0200

0300

0400

0500

0600

0700

0800

0900

1000

1100

1200

1300

1400

$\begin{array}{ll}10.6 & 170 \\ 10.9 & 170 \\ 11.1 & 170 \\ 11.2 & 170\end{array}$

169

170

169

169

170

170

170

171

171

171

171

170

170

170

168

167

164

160

162

163

163

$\begin{array}{rr}10.1 & 90 \\ 9.7 & 87 \\ 9.4 & 85 \\ 9.4 & 85 \\ 9.4 & 85 \\ 9.4 & 85 \\ 9.5 & 86 \\ 9.6 & 87 \\ 9.7 & 88 \\ 9.8 & 89 \\ 9.9 & 90\end{array}$

9.9

10.1

10.1

10.2

10.3

10.3

10.4

10.4

10.5

10.3

10.0

9.7

9.7

9.7
90

87

85

85

85

85

86

87

88

89

90

90

91

91

92

93

93

93

93

95

94

92

91

91

92
8.1

8.1

8.1

8.1

8.1

8.1

8.1

8.0

8.0

8.0

8.1

8.1

8.1

8.1

8.0

8.1

8.1

8.1

8.1

8.1

8.1

8.0

8.0

8.0

8.0
--

--

--

-.

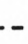

-

$-$

-. 
Site number and name: 15 - South Fork John Day River at Dayville

Date: August 12-13, 1977

Discharge $\left(\mathrm{ft}^{3} / \mathrm{s}\right): 1.9$

\begin{tabular}{|c|c|c|c|c|c|c|}
\hline $\begin{array}{l}\text { Time } \\
(2400 \\
\text { hours })\end{array}$ & $\begin{array}{l}\text { Water } \\
\text { tempera- } \\
\text { ture } \\
\left({ }^{\circ} \mathrm{C}\right)\end{array}$ & $\begin{array}{l}\text { Specific } \\
\text { conductance } \\
\text { (micromhos/ } \\
\mathrm{cm} \text { at } 25^{\circ} \mathrm{C} \text { ) }\end{array}$ & $\begin{array}{l}\text { Dissolved } \\
\text { oxygen } \\
(\mathrm{mg} / \mathrm{L})\end{array}$ & $\begin{array}{l}\text { Dissolved } \\
\text { oxygen } \\
\text { (percent } \\
\text { saturation) }\end{array}$ & $\begin{array}{c}\mathrm{pH} \\
\text { (units) }\end{array}$ & $\begin{array}{c}\text { Pyrometer } \\
\text { (calories/ } \\
\mathrm{cm}^{2} / \mathrm{min} \text { ) }\end{array}$ \\
\hline
\end{tabular}

$\frac{\text { Aug. } 12}{1300}$

1400

1500

24.3

25.5

26.1

1600

26.1

445

450

450

448

11.0

10.4

10.0

9.7

130

125

122

118

8.1

8.2

8.2

440

1700

25.8

9.2

112

8.5

7.4

435

1900

23.3

440

2000

22.5

21.5

444

6.7

101

86

77

70

8.2

448

2200

20.8

452

2400

19.9

454

64

5.8

5.6

61

59

8.2

8.2

8.1

8.0

7.9

Aug. 13

$18.4 \quad 455$

5.5

7.9

7.9

5.4

57

7.8

0100

455

0200

17.7

455

0300

17.0

455

0400

16.3

458

0500

15.7

460

0600

15.0

458

5.3

5.3

5.4

5.4

5.5

55

54

55

54

54

7.8

0700

14.6

460

0800

456

6.0

59

7.4

73

455

1000

15.7

9.2

92

457

10.5

108

7.8

7.8

7.8

7.9

7.9

1100

17.3

460

11.4

124

8.0

8.0

8.1

8.3

8.4 
Site number and name: 15 - South Fork John Day River at Dayville

Date: September 6-7, 1977

Discharge $\left(\mathrm{ft}^{3} / \mathrm{s}\right): 2.1$

\begin{tabular}{|c|c|c|c|c|c|c|}
\hline $\begin{array}{l}\text { Time } \\
(2400 \\
\text { hours })\end{array}$ & $\begin{array}{l}\text { Water } \\
\text { tempera- } \\
\text { ture } \\
\left({ }^{\circ} \mathrm{C}\right)\end{array}$ & $\begin{array}{l}\text { Specific } \\
\text { conductance } \\
\text { (micromhos/ } \\
\mathrm{cm} \text { at } 25^{\circ} \mathrm{C} \text { ) }\end{array}$ & $\begin{array}{l}\text { Dissolved } \\
\text { oxygen } \\
(\mathrm{mg} / \mathrm{L})\end{array}$ & $\begin{array}{l}\text { Dissolved } \\
\text { oxygen } \\
\text { (percent } \\
\text { saturation) }\end{array}$ & $\begin{array}{c}\mathrm{pH} \\
\text { (units) }\end{array}$ & $\begin{array}{c}\text { Pyrometer } \\
\text { (calories/ } \\
\mathrm{cm}^{2} / \mathrm{min} \text { ) }\end{array}$ \\
\hline
\end{tabular}

\section{$\frac{\text { Sept. } 6}{1900}$}

2000

2100

2200

2300

2400

$\frac{\text { Sept. } 7}{0100}$

0200

0300

0400

0500

0600

0700

0800

0900

1000

1100

1200

1300

1400

1500

1600

1700

1800

$\begin{array}{ll}22.8 & 376 \\ 22.2 & 378 \\ 21.7 & 382 \\ 20.8 & 388\end{array}$

$$
20.1
$$

19.2

395

400

18.7

17.9

17.3

16.3

15.6

14.9

14.0

14.0

14.7

15.3

17.1

19.2

20.2

21.8

22.8

22.2

22.0

21.3

$\begin{array}{rr}8.2 & 102 \\ 7.5 & 93 \\ 6.4 & 78 \\ 6.0 & 72\end{array}$

$5.7 \quad 68$

$5.6 \quad 65$

5.9

6.0

6.1

6.3

6.5

6.7

7.0

7.4

8.4

10.0

11.5

11.9

11.8

11.8

11.6

11.3

10.7

9.5

102
93
78
72
68
65

68

68

68

69

71

72

74

78

89

109

129

137

140

144

145

141

134

117

$\begin{array}{ll}8.0 & -- \\ 7.9 & -- \\ 7.8 & -- \\ 7.8 & --\end{array}$

7.7

7.7

7.7

7.7

7.7

7.8

7.8

7.8

7.8

7.9 --

8.0 --

8.1 --

8.2

8.3

8.3 --

8.4 --

8.4 --

8.4

8.4

8.3 
Site number and name: 15 - South Fork John Day River at Dayville

Date: September 13-15, 1978

Discharge $\left(\mathrm{ft}^{3} / \mathrm{s}\right): 43$

\begin{tabular}{|c|c|c|c|c|c|c|}
\hline $\begin{array}{l}\text { Time } \\
(2400 \\
\text { hours })\end{array}$ & $\begin{array}{l}\text { Water } \\
\text { tempera- } \\
\text { ture } \\
\left({ }^{\circ} \mathrm{C}\right)\end{array}$ & $\begin{array}{l}\text { Specific } \\
\text { conductance } \\
\text { (micromhos/ } \\
\mathrm{cm} \text { at } 25^{\circ} \mathrm{C} \text { ) }\end{array}$ & $\begin{array}{l}\text { Dissolved } \\
\text { oxygen } \\
\text { (mg/L) }\end{array}$ & $\begin{array}{l}\text { Dissolved } \\
\text { oxygen } \\
\text { (percent } \\
\text { saturation) }\end{array}$ & $\begin{array}{c}\mathrm{pH} \\
\text { (units) }\end{array}$ & $\begin{array}{c}\text { Pyrometer } \\
\text { (calories/ } \\
\mathrm{cm}^{2} / \mathrm{min} \text { ) }\end{array}$ \\
\hline
\end{tabular}

Sept. 13

$1200 \quad 13.5$

$1300 \quad 14.1 \quad 367$

$\begin{array}{lll}1400 & 14.6 & 367\end{array}$

$1500 \quad 14.8 \quad 364$

$\begin{array}{lll}1600 & 14.9 & 359\end{array}$

$1700 \quad 15.0 \quad 357$

$1800 \quad 15.0 \quad 356$

$\begin{array}{lll}1900 & 14.8 & 353\end{array}$

2000

14.7

348

2100

14.4

14.2

14.0

343

2200

13.8

343

2400

Sept. 14

0100

13.7

13.6

13.4

13.4

0400

13.4

11.0

105

8.2

0.42

10.9

8.5

.42

10.9

105

10.6

104

8.5

.38

$10.7 \quad 105$

$10.6 \quad 104$

$10.3 \quad 101$

$10.0 \quad 98$

9.492

$9.2 \quad 89$

9.2

9.3

9.4

89

89
90

90

$8.6 \quad .20$

$8.5 \quad .22$

$8.4 \quad .15$

$8.3 \quad .10$

$8.2 \quad 0^{.02}$

0500

13.2

340

9.5

9.5

9.6

9.6

9.6

$8.0 \quad 0$

0600

0700

13.2

9.6

9.6

8.0

7.9

7.8

0

0900

13.3

9.6

9.9

10.6

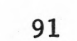

7.8
7.8

7.8
7.8

7.8

7.8

7.8

7.8

7.9

.02

1000

13.8

367

1100

14.5

365

10.9

11.1

11.1

11.2

8.0

.08

.22

1300

16.3

366
373

1400

16.7

369

1500

17.3

363

1600

17.5

365

1700

1800

17.5

366

$17.2 \quad 366$

2000

16.8

367

367

11.2

8.3

8.4

.84

.52

$8.4 \quad .48$

10.8

$10.8 \quad 112$

$8.4 \quad .68$

$\begin{array}{ll}10.4 & 108 \\ 10.0 & 103\end{array}$

$9.6 \quad 98$

9.293

8.4

$8.4-74$

$8.3 \quad .16$

$8.3 \quad .02$

$9.1 \quad 91$

9.1

9.3

8.2

2200

15.6

366

2300
2400

14.5

367

Sept. 15

$14.0 \quad 365$

$\begin{array}{lll}0100 & 13.6 & 364\end{array}$

$0200 \quad 13.1 \quad 362$

$0300 \quad 12.8-362$

$\begin{array}{lll}0400 & 12.5 & 363\end{array}$

0500

12.4

363
364

$0600 \quad 12.3 \quad 364$

$0700 \quad 12.4 \quad 366$

0800

12.5

366
369

9.5

9.6

9.7

9.7

9.7

8.1

8.1

8.0

7.9

0

90

91

9.7

9.7
10.1

7.8
7.8

7.8

7.9

7.9

7.9

7.9
8.0

90
90
94

.02
.12 
Site number and name: 16 - Pearson Creek, Umatilla River basin

Date: September 20-21, 1978

Discharge $\left(\mathrm{ft}^{3} / \mathrm{s}\right): 1.8$

\begin{tabular}{|c|c|c|c|c|c|c|}
\hline $\begin{array}{l}\text { Time } \\
(2400 \\
\text { hours })\end{array}$ & $\begin{array}{l}\text { Water } \\
\text { tempera- } \\
\text { ture } \\
\left({ }^{\circ} \mathrm{C}\right)\end{array}$ & $\begin{array}{l}\text { Specific } \\
\text { conductance } \\
\text { (micromhos/ } \\
\mathrm{cm} \text { at } 25^{\circ} \mathrm{C} \text { ) }\end{array}$ & $\begin{array}{c}\text { Dissolved } \\
\text { oxygen } \\
(\mathrm{mg} / \mathrm{L})\end{array}$ & $\begin{array}{l}\text { Dissolved } \\
\text { oxygen } \\
\text { (percent } \\
\text { saturation) }\end{array}$ & $\begin{array}{c}\mathrm{pH} \\
\text { (units) }\end{array}$ & $\begin{array}{c}\text { Pyrometer } \\
\text { (calories/ } \\
\mathrm{cm}^{2} / \mathrm{min} \text { ) }\end{array}$ \\
\hline \multicolumn{7}{|c|}{ Sept. 20} \\
\hline 1200 & 7.4 & 99 & 10.9 & 90 & 7.8 & 0.56 \\
\hline 1300 & 8.4 & 100 & 10.7 & 91 & 7.8 & .56 \\
\hline 1400 & 9.4 & 103 & 10.4 & 91 & 7.9 & .12 \\
\hline 1500 & 9.3 & 104 & 10.1 & 88 & 7.8 & .12 \\
\hline 1600 & 9.1 & 104 & 10.1 & 87 & 7.8 & .14 \\
\hline 1700 & 8.9 & 104 & 10.1 & 87 & 7.7 & .08 \\
\hline 1800 & 8.5 & 104 & 10.0 & 85 & 7.6 & .02 \\
\hline 1900 & 8.2 & 104 & 9.9 & 84 & 7.4 & 0 \\
\hline 2000 & 8.0 & 104 & 10.0 & 84 & 7.3 & 0 \\
\hline 2100 & 7.7 & 103 & 10.0 & 84 & 7.3 & 0 \\
\hline 2200 & 7.5 & 103 & 10.1 & 84 & 7.2 & 0 \\
\hline 2300 & 7.3 & 102 & 10.1 & 84 & 7.2 & 0 \\
\hline 2400 & 7.0 & 102 & 10.2 & 84 & 7.2 & 0 \\
\hline \multicolumn{7}{|c|}{ Sept. 21} \\
\hline 0100 & 6.8 & 101 & 10.2 & 83 & 7.2 & 0 \\
\hline 0200 & 6.6 & 100 & 10.2 & 83 & 7.2 & 0 \\
\hline 0300 & 6.5 & 100 & 10.2 & 83 & 7.3 & 0 \\
\hline 0400 & 6.4 & 100 & 10.4 & 84 & 7.4 & 0 \\
\hline 0500 & 6.3 & 100 & 10.4 & 84 & 7.4 & 0 \\
\hline 0600 & 6.3 & 100 & 10.5 & 85 & 7.4 & 0 \\
\hline 0700 & 6.4 & 100 & 10.3 & 83 & 7.4 & .02 \\
\hline 0800 & 6.6 & 100 & 10.4 & 85 & 7.5 & .06 \\
\hline 0900 & 6.8 & 100 & 10.7 & 87 & 7.6 & .12 \\
\hline 1000 & 7.1 & 99 & 10.6 & 87 & 7.7 & .12 \\
\hline 1100 & 7.4 & 99 & 10.6 & 88 & 7.8 & .18 \\
\hline
\end{tabular}


Site number and name: 17 - East Fork Birch Creek, Umatilla River basin

Date: September 21-22, 1978

Discharge $\left(\mathrm{ft}^{3} / \mathrm{s}\right): 5.0$

\begin{tabular}{|c|c|c|c|c|c|c|}
\hline $\begin{array}{l}\text { Time } \\
(2400 \\
\text { hours })\end{array}$ & $\begin{array}{l}\text { Water } \\
\text { tempera- } \\
\text { ture } \\
\left({ }^{\circ} \mathrm{C}\right)\end{array}$ & $\begin{array}{l}\text { Specific } \\
\text { conductance } \\
\text { (micromhos/ } \\
\mathrm{cm} \text { at } 25^{\circ} \mathrm{C} \text { ) }\end{array}$ & $\begin{array}{c}\text { Dissolved } \\
\text { oxygen } \\
\text { (mg/L) }\end{array}$ & $\begin{array}{l}\text { Dissolved } \\
\text { oxygen } \\
\text { (percent } \\
\text { saturation) }\end{array}$ & $\underset{\text { (units) }}{\mathrm{pH}}$ & $\begin{array}{c}\text { Pyrometer } \\
\text { (calories/ } \\
\mathrm{cm}^{2} / \mathrm{min} \text { ) }\end{array}$ \\
\hline
\end{tabular}

\begin{tabular}{|c|c|c|}
\hline \multicolumn{3}{|c|}{ Sept. 21} \\
\hline 1300 & 13.1 & 183 \\
\hline 1400 & 13.7 & 184 \\
\hline 1500 & 14.5 & 187 \\
\hline 1600 & 15.3 & 189 \\
\hline 1700 & 15.2 & 191 \\
\hline 1800 & 15.0 & 191 \\
\hline 1900 & 14.6 & 192 \\
\hline 2000 & 14.2 & 191 \\
\hline 2100 & 13.9 & 192 \\
\hline 2200 & 13.6 & 191 \\
\hline 2300 & 13.3 & 189 \\
\hline 2400 & 13.1 & 188 \\
\hline \multicolumn{3}{|c|}{ Sept. 22} \\
\hline 0100 & 12.8 & 187 \\
\hline 0200 & 12.6 & 186 \\
\hline 0300 & 12.6 & 185 \\
\hline 0400 & 12.4 & 184 \\
\hline 0500 & 12.3 & 183 \\
\hline 0600 & 12.2 & 183 \\
\hline 0700 & 12.0 & 181 \\
\hline 0800 & 11.9 & 181 \\
\hline 0900 & 12.3 & 181 \\
\hline 1000 & 12.5 & 181 \\
\hline 1100 & 13.1 & 182 \\
\hline
\end{tabular}

$\begin{array}{rrrl}11.0 & 104 & 7.8 & 0.16 \\ 10.6 & 102 & 7.8 & .34 \\ 10.2 & 99 & 7.9 & .50 \\ 9.6 & 95 & 7.9 & .22 \\ 9.1 & 90 & 7.8 & .14 \\ 8.8 & 87 & 7.6 & .04 \\ 8.5 & 83 & 7.4 & 0 \\ 8.6 & 83 & 7.4 & 0 \\ 8.7 & 84 & 7.4 & 0 \\ 8.9 & 85 & 7.3 & 0 \\ 9.1 & 86 & 7.3 & 0 \\ 9.2 & 87 & 7.3 & 0 \\ 9.3 & 87 & 7.3 & 0 \\ 9.5 & 89 & 7.3 & 0 \\ 9.5 & 89 & 7.3 & 0 \\ 9.6 & 89 & 7.3 & 0 \\ 9.6 & 89 & 7.3 & 0 \\ 9.7 & 90 & 7.3 & 0 \\ 9.8 & & & \\ 10.1 & 90 & 7.3 & .04 \\ 10.5 & 93 & 7.4 & .12 \\ 10.4 & 98 & 7.5 & .18 \\ 10.5 & 97 & 7.6 & .20 \\ & 99 & 7.7 & .40\end{array}$


Site number and name: 18 - Wildhorse Creek, Umatilla River basin

Date: September 22-23, 1978

Discharge $\left(\mathrm{ft}^{3} / \mathrm{s}\right): 0.05$

\begin{tabular}{|c|c|c|c|c|c|c|}
\hline $\begin{array}{l}\text { Time } \\
(2400 \\
\text { hours })\end{array}$ & $\begin{array}{l}\text { Water } \\
\text { tempera- } \\
\text { ture } \\
\left({ }^{\circ} \mathrm{C}\right)\end{array}$ & $\begin{array}{l}\text { Specific } \\
\text { conductance } \\
\text { (micromhos/ } \\
\mathrm{cm} \text { at } 25^{\circ} \mathrm{C} \text { ) }\end{array}$ & $\begin{array}{l}\text { Dissolved } \\
\text { oxygen } \\
\text { (mg/L) }\end{array}$ & $\begin{array}{l}\text { Dissolved } \\
\text { oxygen } \\
\text { (percent } \\
\text { saturation) }\end{array}$ & $\begin{array}{c}\mathrm{pH} \\
\text { (units) }\end{array}$ & $\begin{array}{l}\text { Pyrometer } \\
\text { (calories/ } \\
\mathrm{cm}^{2} / \mathrm{min} \text { ) }\end{array}$ \\
\hline
\end{tabular}

\begin{tabular}{cccc} 
Sept. 22 & & \\
\cline { 1 - 1 } 1500 & & 14.3 & 271 \\
1600 & & 14.3 & 271 \\
1700 & & 14.6 & 271 \\
1800 & 14.4 & 271 \\
& & \\
1900 & 14.2 & 271 \\
2000 & 13.8 & 271 \\
2100 & 13.6 & 271 \\
2200 & 13.3 & 271 \\
2300 & 13.2 & 271 \\
& & & \\
2400 & 12.6 & 267 \\
Sept. 23 & & \\
\hline 0100 & 12.0 & 264 \\
0200 & 11.7 & 262 \\
0300 & 11.6 & 262 \\
& & \\
0400 & 11.7 & 262 \\
0500 & 11.8 & 262 \\
0600 & 11.8 & 262 \\
0700 & 11.9 & 263 \\
0800 & 12.1 & 265 \\
& & \\
0900 & 12.4 & 266 \\
1000 & 13.2 & 269 \\
1100 & 14.2 & 274
\end{tabular}

$\begin{array}{rrrl}12.0 & 117 & 8.3 & 0.14 \\ 12.0 & 117 & 8.3 & .12 \\ 12.1 & 118 & 8.4 & .08 \\ 10.9 & 106 & 8.4 & .02 \\ 10.2 & 99 & 8.4 & 0 \\ 10.2 & 98 & 8.3 & 0 \\ 9.5 & 91 & 8.2 & 0 \\ 9.2 & 87 & 8.2 & 0 \\ 8.9 & 84 & 8.1 & 0 \\ 8.7 & 81 & 8.0 & 0 \\ 8.5 & 78 & 8.0 & 0 \\ 8.4 & 77 & 8.0 & 0 \\ 8.1 & 74 & 8.0 & 0 \\ 8.5 & & & \\ 9.8 & 78 & 8.0 & 0 \\ 9.8 & 90 & 7.9 & 0 \\ 9.2 & 90 & 7.9 & 0 \\ 9.1 & 85 & 7.9 & .02 \\ 9.2 & 84 & 7.9 & .06 \\ 9.1 & & & \\ 9.5 & 86 & 7.9 & .32 \\ 96 & 92 & 8.0 & .70 \\ & & 8.0 & .68\end{array}$


Table 3.--Chemical data

[n.a., not analyzed. A11 constituents dissolved except organic carbon, nitrogen species, and total phosphorus]

\begin{tabular}{|c|c|c|c|c|c|c|c|}
\hline \multicolumn{8}{|c|}{ Site number $\underline{1}$ (Evans Creek basin) } \\
\hline & IA & $1 B$ & 2 & 2 & 2 & 3 & 3 \\
\hline $\begin{array}{l}\text { Date } \\
\text { Time } \\
\text { Discharge }\left(\mathrm{ft}^{3} / \mathrm{s}\right)\end{array}$ & $\begin{array}{r}8-11-77 \\
1800 \\
0.25\end{array}$ & $\begin{array}{r}7-6-78 \\
1530 \\
5.9\end{array}$ & $\begin{array}{r}8-11-77 \\
1630 \\
3.4\end{array}$ & $\begin{array}{r}9-8-77 \\
1200 \\
4.7\end{array}$ & $\begin{array}{r}7-7-78 \\
1100 \\
20\end{array}$ & $\begin{array}{r}9-7-77 \\
1200 \\
2.6\end{array}$ & $\begin{array}{r}7-5-78 \\
1350 \\
28\end{array}$ \\
\hline \multicolumn{8}{|c|}{ Dissolved constituents (mg/L) } \\
\hline $\begin{array}{l}\text { Calcium } \\
\text { Carbon, organic } \\
\text { Chloride } \\
\text { Hardness (Ca, Mg) } \\
\text { Iron } \\
\text { Magnesium } \\
\text { Manganese } \\
\text { Nitrogen as N } \\
\quad \text { Ammonia } \\
\quad \text { Kjeldah1 } \\
\quad \text { Nitrite + } \\
\quad \text { nitrate } \\
\text { Phosphorus as P } \\
\quad \text { Total } \\
\quad \text { Orthophosphate } \\
\text { Potassium } \\
\text { Silica } \\
\text { Sodium } \\
\text { Sulfate }\end{array}$ & $\begin{array}{l}12 \\
2.4 \\
2.0 \\
48 \\
70 \\
4.3 \\
0 \\
\\
\\
.01 \\
.14 \\
.01\end{array}$ & $\begin{array}{l}16 \\
1.9 \\
2.0 \\
65 \\
40 \\
6.2 \\
10\end{array}$ & $\begin{array}{l}12 \\
1.5 \\
28 \\
57 \\
70 \\
6.6 \\
20\end{array}$ & $\begin{array}{l}23 \\
1.7 \\
29 \\
85 \\
70 \\
6.6 \\
20 \\
\\
.02 \\
.05 \\
.01 \\
\\
.02 \\
.02 \\
1.4 \\
21 \\
22 \\
2.3\end{array}$ & $\begin{array}{l}14 \\
1.7 \\
13 \\
63 \\
50 \\
6.7 \\
0 \\
\\
.00 \\
.09 \\
.00\end{array}$ & $\begin{array}{l}21 \\
1.6 \\
21 \\
86 \\
90 \\
8.1 \\
50 \\
\\
.03 \\
.17 \\
.04 \\
\\
.02 \\
.01 \\
1.6 \\
23 \\
16 \\
4.1\end{array}$ & $\begin{array}{l}14 \\
1.7 \\
8.1 \\
64 \\
40 \\
7.0 \\
0 \\
\\
.00 \\
.00 \\
.05 \\
\\
\\
.02 \\
.01 \\
1.0 \\
17 \\
9.2 \\
2.1\end{array}$ \\
\hline \multicolumn{8}{|c|}{ Alkalinity } \\
\hline $\begin{array}{l}\text { Date } \\
\text { Time } \\
\text { Mg/L }\end{array}$ & $\begin{array}{r}8-11-77 \\
0750 \\
85\end{array}$ & $\begin{array}{r}7-6-78 \\
1445 \\
72\end{array}$ & $\begin{array}{r}8-11-77 \\
2045 \\
65\end{array}$ & $\begin{array}{r}9-8-77 \\
1445 \\
75\end{array}$ & $\begin{array}{r}7-6-78 \\
1730 \\
67\end{array}$ & $\begin{array}{r}9-7-77 \\
1400 \\
166\end{array}$ & $\begin{array}{r}7-5-78 \\
1420 \\
67\end{array}$ \\
\hline $\begin{array}{l}\text { Date } \\
\text { Time } \\
\mathrm{Mg} / \mathrm{L}\end{array}$ & $\begin{array}{r}8-12-77 \\
1315 \\
85\end{array}$ & $\begin{array}{r}7-6-78 \\
1825 \\
80\end{array}$ & $\begin{array}{r}8-12-77 \\
0720 \\
73\end{array}$ & $\begin{array}{r}9-8-77 \\
1930 \\
74\end{array}$ & $\begin{array}{r}7-7-78 \\
0810 \\
71\end{array}$ & $\begin{array}{r}9-7-77 \\
1940 \\
84\end{array}$ & $\begin{array}{r}7-5-78 \\
1650 \\
67\end{array}$ \\
\hline $\begin{array}{l}\text { Date } \\
\text { Time } \\
\text { Mg/L } \\
\text { Date } \\
\text { Time } \\
\text { Mg/L }\end{array}$ & $\begin{array}{r}8-12-77 \\
2015 \\
84\end{array}$ & $\begin{array}{r}7-7-78 \\
0830 \\
72 \\
7-7-78 \\
1330 \\
74\end{array}$ & $\begin{array}{r}8-12-77 \\
1700 \\
73\end{array}$ & $\begin{array}{r}9-9-77 \\
0745 \\
77\end{array}$ & $\begin{array}{r}7-7-78 \\
1230 \\
66\end{array}$ & $\begin{array}{r}9-8-78 \\
0800 \\
87\end{array}$ & $\begin{array}{r}7-6-78 \\
0740 \\
71\end{array}$ \\
\hline
\end{tabular}

See footnote at end of table. 
Table 3.--Chemical data--Continued

\begin{tabular}{|c|c|c|c|c|}
\hline & \multicolumn{4}{|c|}{ Site number $1 /$ (Molalla River basin) } \\
\hline & 4 & 5 & 6 & 7 \\
\hline $\begin{array}{l}\text { Date } \\
\text { Time } \\
\text { Discharge }\left(\mathrm{ft}^{3} / \mathrm{s}\right)\end{array}$ & $\begin{array}{r}7-21-77 \\
1200 \\
6.0\end{array}$ & $\begin{array}{r}9-15-77 \\
1200 \\
3.2\end{array}$ & $\begin{array}{r}9-14-77 \\
1200 \\
0.14\end{array}$ & $\begin{array}{r}9-13-77 \\
1200 \\
19\end{array}$ \\
\hline \multicolumn{5}{|c|}{ Dissolved constituents (mg/L) } \\
\hline $\begin{array}{l}\text { Calcium } \\
\text { Carbon } \\
\text { Chloride } \\
\text { Hardness (Ca, Mg) } \\
\text { Iron } \\
\text { Magnesium } \\
\text { Manganese } \\
\text { Nitrogen as N } \\
\quad \text { Ammonia } \\
\quad \text { Kjeldahl } \\
\quad \text { Nitrite }+ \\
\quad \text { nitrate } \\
\\
\text { Phosphorus as P } \\
\quad \text { Total } \\
\quad \text { Orthophosphate } \\
\\
\text { Potassium } \\
\text { Silica } \\
\text { Sodium } \\
\text { Sulfate }\end{array}$ & $\begin{array}{l}6.0 \\
.6 \\
1.0 \\
20 \\
40 \\
1.2 \\
0 \\
\\
.01 \\
.08 \\
.02\end{array}$ & $\begin{array}{l}6.1 \\
.3 \\
2.6 \\
20 \\
20 \\
1.1 \\
0 \\
\\
.00 \\
.00 \\
.11\end{array}$ & $\begin{array}{l}5.8 \\
.6 \\
3.2 \\
31 \\
100 \\
3.9 \\
0 \\
\\
.00 \\
.37 \\
.30\end{array}$ & $\begin{array}{c}6.6 \\
1.5 \\
3.6 \\
30 \\
370 \\
3.3 \\
20 \\
\\
\\
.04 \\
.14 \\
.07\end{array}$ \\
\hline \multicolumn{5}{|c|}{ Alkalinity } \\
\hline $\begin{array}{l}\text { Date } \\
\text { Time } \\
\text { Mg/L }\end{array}$ & $\begin{array}{r}7-21-77 \\
1700 \\
1.0\end{array}$ & $\begin{array}{r}9-15-77 \\
1430 \\
23\end{array}$ & $\begin{array}{r}9-14-77 \\
1410 \\
34\end{array}$ & $\begin{array}{r}9-13-77 \\
1430 \\
37\end{array}$ \\
\hline $\begin{array}{l}\text { Date } \\
\text { Time } \\
\qquad \mathrm{Mg} / \mathrm{L}\end{array}$ & $\begin{array}{r}7-21-77 \\
2000 \\
.95\end{array}$ & $\begin{array}{r}9-16-77 \\
0825 \\
21\end{array}$ & $\begin{array}{r}9-15-77 \\
0845 \\
34\end{array}$ & $\begin{array}{r}9-13-77 \\
1630 \\
37\end{array}$ \\
\hline $\begin{array}{l}\text { Date } \\
\text { Time } \\
\qquad \mathrm{Mg} / \mathrm{L}\end{array}$ & $\begin{array}{r}7-22-77 \\
0700 \\
1.0\end{array}$ & & & $\begin{array}{r}9-14-78 \\
815 \\
36\end{array}$ \\
\hline $\begin{array}{l}\text { Date } \\
\text { Time } \\
\mathrm{Mg} / \mathrm{L}\end{array}$ & $\begin{array}{r}7-22-77 \\
1300 \\
.9\end{array}$ & & & \\
\hline
\end{tabular}

See footnote at end of table. 
Table 3.--Chemical data--Continued

\begin{tabular}{|c|c|c|c|c|c|c|}
\hline \multicolumn{7}{|c|}{ Site number $\underline{1}$ / (Siuslaw basin area) } \\
\hline & 8 & 9 & 10 & 10 & 11 & 11 \\
\hline $\begin{array}{l}\text { Date } \\
\text { Time } \\
\text { Discharge }\left(\mathrm{ft}^{3} / \mathrm{s}\right)\end{array}$ & $\begin{array}{r}10-4-77 \\
1000 \\
8.5\end{array}$ & $\begin{array}{r}10-4-77 \\
1200 \\
2.7\end{array}$ & $\begin{array}{r}8-9-77 \\
1600 \\
0.43\end{array}$ & $\begin{array}{r}10-5-77 \\
1200 \\
2.4\end{array}$ & $\begin{array}{r}8-9-77 \\
1130 \\
2.7\end{array}$ & $\begin{array}{r}10-5-77 \\
1200 \\
9.2\end{array}$ \\
\hline \multicolumn{7}{|c|}{ Dissolved constituents (mg/L) } \\
\hline $\begin{array}{l}\text { Calcium } \\
\text { Carbon } \\
\text { Chloride } \\
\text { Hardness (Ca, Mg) } \\
\text { Iron } \\
\text { Magnesium } \\
\text { Manganese } \\
\text { Nitrogen as N } \\
\quad \text { Ammonia } \\
\quad \text { Kjeldahl } \\
\quad \text { Nitrite + } \\
\quad \text { nitrate } \\
\text { Phosphorus as P } \\
\quad \text { Total } \\
\text { Orthophosphate } \\
\text { Potassium } \\
\text { Silica } \\
\text { Sodium } \\
\text { Sulfate }\end{array}$ & $\begin{array}{l}3.3 \\
1.9 \\
5.3 \\
12 \\
90 \\
1.0 \\
0 \\
\\
.01 \\
.01 \\
.41\end{array}$ & $\begin{array}{l}5.3 \\
2.0 \\
6.9 \\
19 \\
60 \\
1.4 \\
0 \\
\\
\\
.08 \\
.18 \\
.70\end{array}$ & $\begin{array}{l}5.0 \\
1.4 \\
3.1 \\
16 \\
70 \\
.9 \\
0 \\
\\
.01 \\
.09 \\
.12\end{array}$ & $\begin{array}{l}2.5 \\
.8 \\
7.8 \\
11 \\
70 \\
1.1 \\
0 \\
\\
.00 \\
.01 \\
.59\end{array}$ & $\begin{array}{r}.01 \\
.01 \\
12^{.5} \\
7.5 \\
12\end{array}$ & $\begin{array}{c}4.8 \\
3.1 \\
8.4 \\
19 \\
530 \\
1.6 \\
0 \\
\\
\\
.05 \\
.08 \\
.03\end{array}$ \\
\hline \multicolumn{7}{|c|}{ Alkalinity } \\
\hline $\begin{array}{l}\text { Date } \\
\text { Time } \\
\qquad \mathrm{Mg} / \mathrm{L}\end{array}$ & $\begin{array}{r}10-4-77 \\
1600 \\
21\end{array}$ & $\begin{array}{r}10-4-77 \\
1330 \\
18\end{array}$ & $\begin{array}{r}8-8-77 \\
2045 \\
59\end{array}$ & $\begin{array}{r}10-5-77 \\
0900 \\
46\end{array}$ & $\begin{array}{r}8-8-77 \\
2210 \\
22\end{array}$ & $\begin{array}{r}10-5-77 \\
0815 \\
18\end{array}$ \\
\hline $\begin{array}{l}\text { Date } \\
\text { Time } \\
\text { Mg/L } \\
\text { Date } \\
\text { Time } \\
\text { Mg/L }\end{array}$ & $\begin{array}{r}10-5-77 \\
0845 \\
28\end{array}$ & $\begin{array}{r}10-5-77 \\
0800 \\
23\end{array}$ & $\begin{array}{r}8-9-77 \\
0730 \\
52 \\
8-9-77 \\
1340 \\
36\end{array}$ & & $\begin{array}{r}8-9-77 \\
0852 \\
13 \\
8-9-77 \\
1400 \\
11\end{array}$ & \\
\hline
\end{tabular}

See footnote at end of table. 


\begin{tabular}{|c|c|c|c|c|c|c|}
\hline \multicolumn{7}{|c|}{ Site number $1 /$ (South Fork John Day River basin) } \\
\hline & 12 & 13 & 14 & 15 & 15 & 15 \\
\hline $\begin{array}{l}\text { Date collected } \\
\text { Time } \\
\text { Discharge }\left(\mathrm{ft}^{3} / \mathrm{s}\right)\end{array}$ & $\begin{array}{r}9-15-78 \\
1400 \\
0.5\end{array}$ & $\begin{array}{r}9-15-78 \\
1500 \\
16\end{array}$ & $\begin{array}{r}9-14-78 \\
1130 \\
9.1\end{array}$ & $\begin{array}{r}8-12-77 \\
1400 \\
1.9\end{array}$ & $\begin{array}{r}9-7-77 \\
0930 \\
2.1\end{array}$ & $\begin{array}{r}9-14-78 \\
1400 \\
43\end{array}$ \\
\hline \multicolumn{7}{|c|}{ Dissolved constituents (mg/L) } \\
\hline $\begin{array}{l}\text { Calcium } \\
\text { Carbon } \\
\text { Chloride } \\
\text { Hardness (Ca, Mg) } \\
\text { Iron } \\
\text { Magnesium } \\
\text { Manganese } \\
\text { Nitrogen as N } \\
\quad \text { Ammonia } \\
\quad \text { Kjeldahl } \\
\quad \text { Nitrite }+ \\
\quad \text { nitrate } \\
\\
\text { Phosphorus as P } \\
\quad \text { Total } \\
\text { Orthophosphate } \\
\text { Potassium } \\
\text { Silica } \\
\text { Sodium } \\
\text { Sulfate }\end{array}$ & $\begin{array}{c}45 \\
1.6 \\
1.6 \\
160 \\
20 \\
12 \\
0 \\
\\
.01 \\
.16 \\
.01 \\
\\
\\
.04 \\
.01 \\
1.8 \\
23 \\
12 \\
11\end{array}$ & $\begin{array}{l}94 \\
1.7 \\
3.6 \\
320 \\
20 \\
20 \\
20 \\
\\
.02 \\
.39 \\
.02 \\
\\
\\
.02 \\
.02 \\
\\
2.7 \\
23 \\
32 \\
100\end{array}$ & $\begin{array}{c}20 \\
1.3 \\
.9 \\
79 \\
20 \\
7.1 \\
0 \\
\\
.00 \\
.15 \\
.02\end{array}$ & $\begin{array}{l}n_{0} a_{0} \\
\text { n.a. } \\
\text { n.a. } \\
n_{0} a_{0} \\
n_{0} a_{0} \\
\text { n.a. } \\
\text { n.a. } \\
\\
\text { n.a. } \\
\text { n.a. } \\
0.03\end{array}$ & $\begin{array}{l}\text { n.a. } \\
\text { n.a. } \\
n_{0} a_{0} \\
\text { n.a. } \\
\text { n.a. } \\
\text { n.a. } \\
\text { n.a. } \\
\\
\text { n.a. } \\
\text { n.a. } \\
\text { n.a. }\end{array}$ & $\begin{array}{c}44 \\
2.6 \\
3.1 \\
170 \\
20 \\
14 \\
0 \\
\\
.02 \\
.23 \\
.01 \\
\\
\\
.02 \\
.01 \\
1.9 \\
28 \\
17 \\
34\end{array}$ \\
\hline \multicolumn{7}{|c|}{ Alkalinity } \\
\hline $\begin{array}{l}\text { Date } \\
\text { Time } \\
\mathrm{Mg} / \mathrm{L} \\
\text { Date } \\
\text { Time } \\
\mathrm{Mg} / \mathrm{L} \\
\text { Date } \\
\text { Time } \\
\mathrm{Mg} / \mathrm{L} \\
\text { Date } \\
\text { Time } \\
\mathrm{Mg} / \mathrm{L}\end{array}$ & $\begin{array}{r}9-14-78 \\
1905 \\
180\end{array}$ & $\begin{array}{r}9-15-78 \\
1800 \\
260\end{array}$ & $\begin{array}{r}9-13-78 \\
1400 \\
91.8 \\
\\
9-14-78 \\
0945 \\
89.4\end{array}$ & $\begin{array}{r}8-12-77 \\
1400 \\
210 \\
8-12-77 \\
1930 \\
210 \\
\\
8-13-77 \\
0705 \\
230\end{array}$ & $\begin{array}{r}9-6-77 \\
1930 \\
196 \\
\\
9-7-77 \\
0700 \\
216 \\
\\
9-7-77 \\
1220 \\
207 \\
\\
9-7-77 \\
1445 \\
207\end{array}$ & $\begin{array}{r}9-13-78 \\
1235 \\
171 \\
9-14-78 \\
0900 \\
166\end{array}$ \\
\hline
\end{tabular}

See footnote at end of table. 
Table 3.--Chemical data--Continued

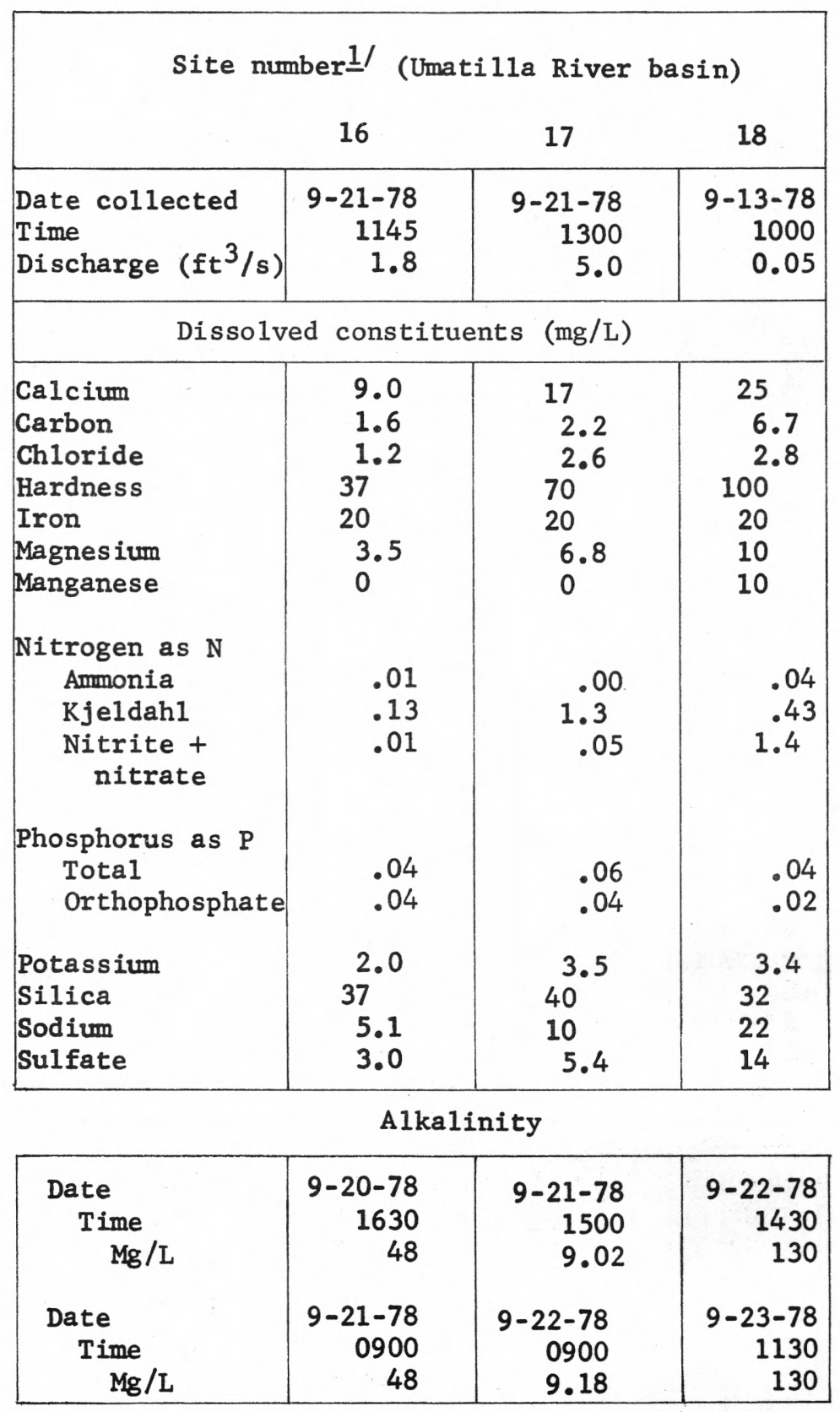

1/ Refer to table 1 for site names. 
Table 4.--Miscellaneous data

[n.a., not analyzed]

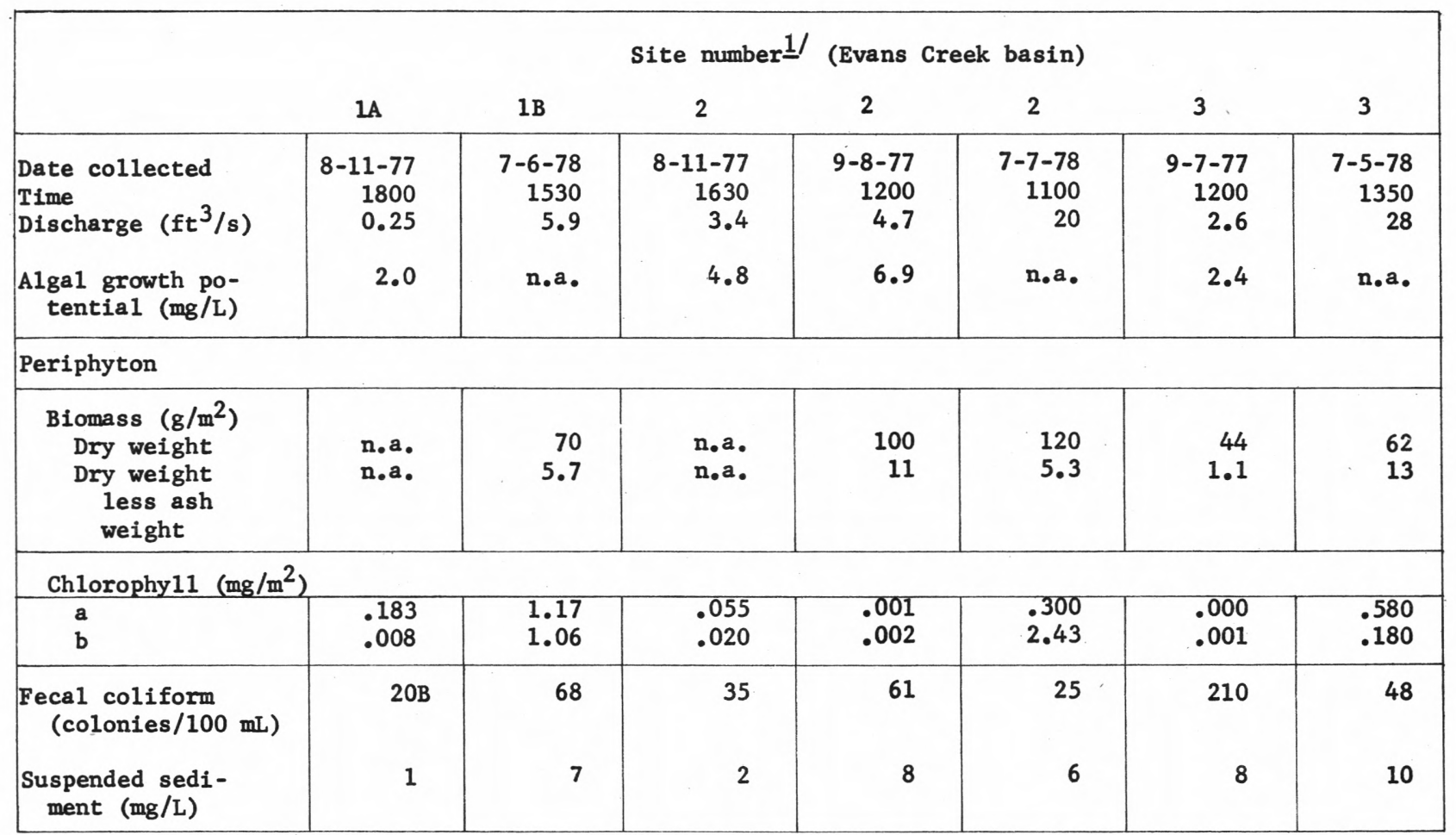

Oxygen, light-dark bottle test

\begin{tabular}{|c|c|c|c|c|c|c|c|}
\hline $\begin{array}{l}\text { Started } \\
\text { Time } \\
\text { Ended } \\
\text { Time }\end{array}$ & $\begin{array}{r}8-11-77 \\
1305 \\
8-12-77 \\
1220\end{array}$ & $\begin{array}{r}7-6-78 \\
1530 \\
7-7-78 \\
1400\end{array}$ & $\begin{array}{l}-- \\
-- \\
-- \\
--\end{array}$ & $\begin{array}{r}9-8-77 \\
1145 \\
9-9-77 \\
1145\end{array}$ & $\begin{array}{r}7-6-78 \\
1400 \\
7-7-78 \\
1230\end{array}$ & $\begin{array}{r}9-7-77 \\
0930 \\
9-8-77 \\
0930\end{array}$ & $\begin{array}{r}7-5-78 \\
1300 \\
7-6-78 \\
1230\end{array}$ \\
\hline \multicolumn{8}{|c|}{$\begin{array}{l}\text { Productivițy } \\
\quad\left(\mathrm{mg} \mathrm{O}_{2} / \mathrm{m}^{3} / \mathrm{hr}\right)\end{array}$} \\
\hline $\begin{array}{l}\text { Gross primary } \\
\text { Net primary } \\
\text { Respiration }\end{array}$ & $\begin{array}{r}43 \\
-22 \\
65\end{array}$ & $\begin{array}{r}0 \\
-44 \\
44\end{array}$ & $\begin{array}{l}\text { n.a. } \\
\text { n.a. } \\
\text { n.a. }\end{array}$ & $\begin{array}{r}0 \\
-33 \\
33\end{array}$ & $\begin{array}{r}0 \\
-49 \\
49\end{array}$ & $\begin{array}{r}8 \\
-21 \\
29\end{array}$ & $\begin{array}{r}0 \\
-51 \\
51\end{array}$ \\
\hline
\end{tabular}

See footnotes at end of table. 
Table 4.--Miscellaneous data--Continued

\begin{tabular}{|c|c|c|c|c|}
\hline \multicolumn{5}{|c|}{ Site number $1 /$ (Mola1la River basin) } \\
\hline & 4 & 5 & 6 & 7 \\
\hline $\begin{array}{l}\text { Date collected } \\
\text { Time } \\
\left.\text { Discharge ( } \mathrm{ft}^{3} / \mathrm{s}\right) \\
\text { Algal growth po- } \\
\text { tential (mg/L) }\end{array}$ & $\begin{array}{r}7-21-77 \\
1200 \\
6.0 \\
.9\end{array}$ & $\begin{array}{r}9-15-77 \\
1200 \\
3.2 \\
3.7\end{array}$ & $\begin{array}{r}9-14-77 \\
1200 \\
0.14 \\
2.8\end{array}$ & $\begin{array}{r}9-13-77 \\
1200 \\
19 \\
6.8\end{array}$ \\
\hline \multicolumn{5}{|l|}{ Periphyton } \\
\hline $\begin{array}{c}\text { Biomass }\left(\mathrm{g} / \mathrm{m}^{2}\right) \\
\text { Dry weight } \\
\text { Dry weight } \\
\text { less ash } \\
\text { weight }\end{array}$ & $\begin{array}{l}\text { n.a. } \\
\text { n.a. }\end{array}$ & $\begin{array}{r}91 \\
2.6\end{array}$ & $\begin{array}{l}160 \\
8.9\end{array}$ & $\begin{array}{r}160 \\
14\end{array}$ \\
\hline \multicolumn{5}{|l|}{ Chlorophyl1 (mg/m $\left./ \mathrm{m}^{2}\right)$} \\
\hline $\begin{array}{l}a \\
b\end{array}$ & $\begin{array}{l}.363 \\
.012\end{array}$ & $\begin{array}{l}.001 \\
.001\end{array}$ & $\begin{array}{l}\text { n.a. } \\
\text { n.a. }\end{array}$ & $\begin{array}{l}\text { n.a. } \\
\text { n.a. }\end{array}$ \\
\hline $\begin{array}{l}\text { Fecal coliform } \\
\quad(\text { colonies } / 100 \mathrm{~mL})\end{array}$ & n.a. & n.a. & 47 & $520 \mathrm{~B}$ \\
\hline $\begin{array}{l}\text { Suspended sedi- } \\
\text { ment (mg/L) }\end{array}$ & 2 & 6 & 5 & 10 \\
\hline
\end{tabular}

Oxygen, 1ight-dark

bottle test

\begin{tabular}{|c|c|c|c|c|}
\hline $\begin{array}{l}\text { Started } \\
\text { Time } \\
\text { Ended } \\
\text { Time }\end{array}$ & $\begin{array}{r}7-21-77 \\
1200 \\
7-22-77 \\
1200\end{array}$ & $\begin{array}{r}9-15-77 \\
1215 \\
9-16-77 \\
1215\end{array}$ & $\begin{array}{r}9-14-77 \\
1210 \\
9-15-77 \\
1010\end{array}$ & $\begin{array}{r}9-13-77 \\
1025 \\
9-14-77 \\
0925\end{array}$ \\
\hline \multicolumn{5}{|l|}{$\begin{array}{l}\text { Productivity } \\
\qquad\left(\mathrm{mg} 0_{2} / \mathrm{m}^{3} / \mathrm{hr}\right)\end{array}$} \\
\hline $\begin{array}{l}\text { Gross primary } \\
\text { Net primary } \\
\text { Respiration }\end{array}$ & $\begin{array}{r}17 \\
-21 \\
38\end{array}$ & $\begin{array}{l}0 \\
0 \\
0\end{array}$ & $\begin{array}{r}0 \\
-18 \\
18\end{array}$ & $\begin{array}{r}0 \\
-17 \\
17\end{array}$ \\
\hline
\end{tabular}

See footnotes at end of table. 
Table 4.--Miscellaneous data--Continued

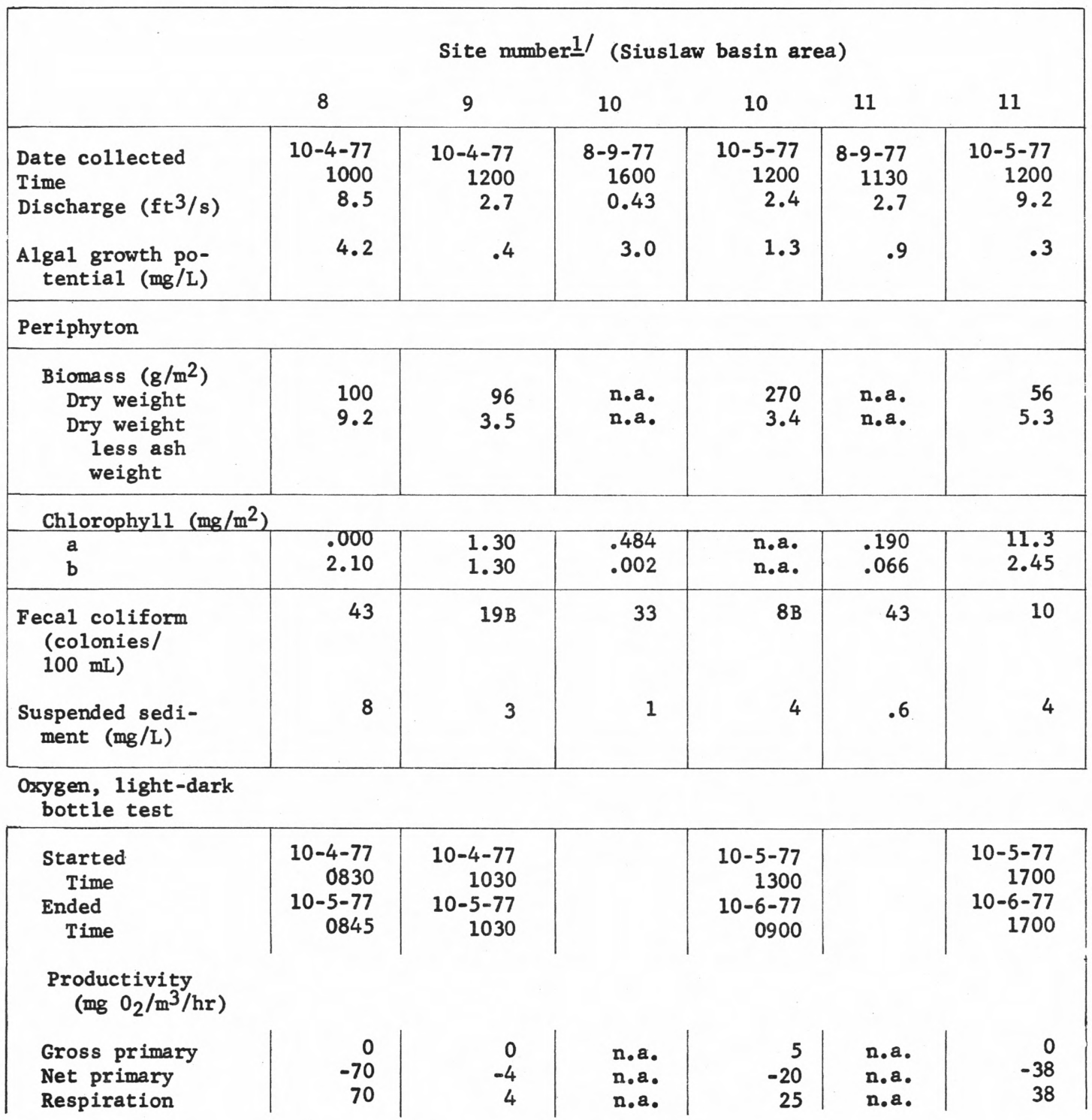

See footnotes at end of table. 
Table 4.--Miscellaneous data--Continued

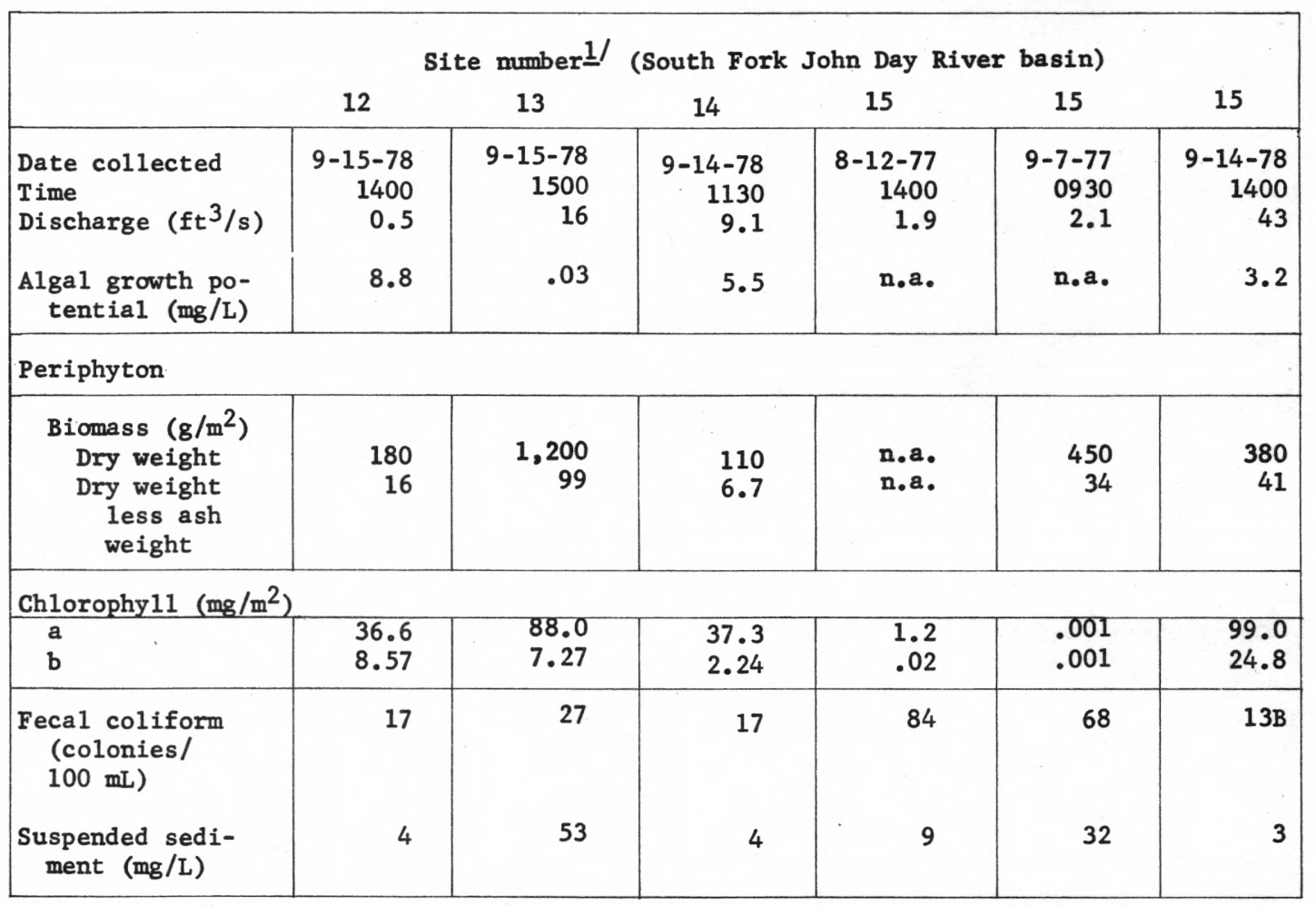

Oxygen, 1ight-dark

bottle test

\begin{tabular}{|c|c|c|c|c|c|c|}
\hline $\begin{array}{l}\text { Started } \\
\text { Time } \\
\text { Ended } \\
\text { Time }\end{array}$ & $\begin{array}{r}9-14-78 \\
1900 \\
9-15-78 \\
1400\end{array}$ & $\begin{array}{r}9-15-78 \\
1000 \\
9-15-78 \\
1700\end{array}$ & & $\begin{array}{r}8-12-77 \\
1305 \\
8-13-77 \\
1045\end{array}$ & $\begin{array}{r}9-6-77 \\
1830 \\
9-7-77 \\
1825\end{array}$ & $\begin{array}{r}9-13-78 \\
1600 \\
9-14-78 \\
1500\end{array}$ \\
\hline \multicolumn{7}{|l|}{$\begin{array}{l}\text { Productivity } \\
\quad\left(\mathrm{mg} \mathrm{O}_{2} / \mathrm{m} 3 / \mathrm{hr}\right)\end{array}$} \\
\hline $\begin{array}{l}\text { Gross primary } \\
\text { Net primary } \\
\text { Respiration }\end{array}$ & $\begin{array}{r}0 \\
-42 \\
42\end{array}$ & $\begin{array}{r}0 \\
-230 \\
230\end{array}$ & $\begin{array}{l}\text { n.a. } \\
\text { n.a. } \\
\text { n.a. }\end{array}$ & $\begin{array}{r}9 \\
-37 \\
46\end{array}$ & $\begin{array}{r}21 \\
21 \\
0\end{array}$ & $\begin{array}{l}\text { n.a. } \\
\text { n.a. } \\
\text { n.a. }\end{array}$ \\
\hline
\end{tabular}

See footnotes at end of table. 
Table 4.--Miscellaneous data--Continued

\begin{tabular}{|c|c|c|c|}
\hline \multicolumn{2}{|c|}{ Site number $\underline{1}$ / } & \multirow{2}{*}{ tilla R } & \multirow[t]{2}{*}{ basin) } \\
\hline \multicolumn{2}{|r|}{16} & & \\
\hline $\begin{array}{l}\text { Date collected } \\
\text { Time } \\
\text { Discharge }\left(\mathrm{ft}^{3} / \mathrm{s}\right)\end{array}$ & $\begin{array}{r}9-21-78 \\
1145 \\
1.8\end{array}$ & $\begin{array}{r}9-21-78 \\
1300 \\
5.0\end{array}$ & $\begin{array}{r}9-23-78 \\
1000 \\
0.05\end{array}$ \\
\hline $\begin{array}{l}\text { Algal growth po- } \\
\text { tential (mg/L) }\end{array}$ & n.a. & 9.6 & 9.5 \\
\hline \multicolumn{4}{|l|}{ Periphyton } \\
\hline $\begin{array}{c}\text { Biomass }\left(\mathrm{g} / \mathrm{m}^{2}\right) \\
\text { Dry weight } \\
\text { Dry weight } \\
\text { less ash } \\
\text { weight }\end{array}$ & $\begin{array}{l}87 \\
13\end{array}$ & $\begin{array}{r}290 \\
28\end{array}$ & $\begin{array}{r}200 \\
24\end{array}$ \\
\hline \multicolumn{4}{|c|}{ Chlorophyl1 (mg/m $\left./ \mathrm{m}^{2}\right)$} \\
\hline $\begin{array}{l}\mathrm{a} \\
\mathrm{b}\end{array}$ & $\begin{array}{r}34.6 \\
.0\end{array}$ & $\begin{array}{r}118 \\
3.87\end{array}$ & $\begin{array}{l}\text { n.a. } \\
\text { n.a. }\end{array}$ \\
\hline $\begin{array}{l}\text { Fecal coliform } \\
\text { (colonies/ } \\
100 \mathrm{~mL} \text { ) }\end{array}$ & 4 & 25 & 9 \\
\hline $\begin{array}{l}\text { Suspended sedi- } \\
\text { ment }(\mathrm{mg} / \mathrm{L})\end{array}$ & 2 & 10 & 6 \\
\hline
\end{tabular}

Oxygen, light-dark

bottle test

\begin{tabular}{|l|r|r|r|}
\hline Started & $9-20-78$ & $9-21-78$ & $9-22-78$ \\
Time & 1230 & 1245 & 1450 \\
Ended & $9-21-78$ & $9-22-78$ & $9-23-78$ \\
Time & 1130 & 1300 & 1000 \\
Productivity & & \\
$\quad$ (mg $\left.0_{2} / \mathrm{m}^{3} / \mathrm{hr}\right)$ & & & \\
Gross primary & & & \\
Net primary & 4 & 21 & 0 \\
Respiration & 4 & -12 & -5 \\
\end{tabular}

1/ Refer to table 1 for site names.

2/ Bacteria samples that did not have one or a combination of filters with a colony count within the ideal of 20 to 60 are reported with the remark " $\mathrm{k} . "$ 
Table 5.--Taxa and numbers of periphyton

Site number and name: $1 \mathrm{~A}$ - Evans Creek above West Fork

Date: August 11, 1977

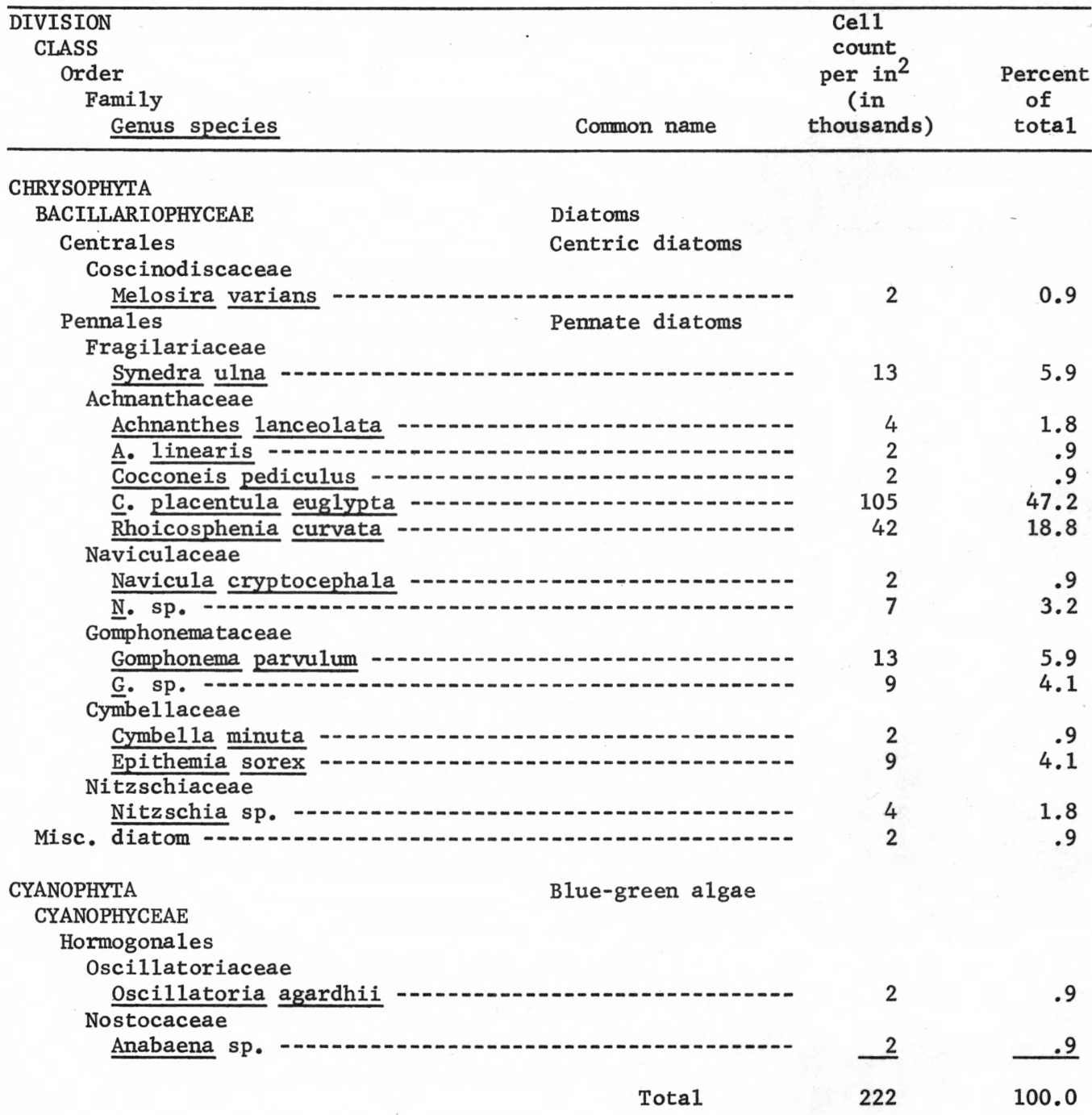


Table 5.--Taxa and numbers of periphyton--Continued

Site number and name: 1B - Evans Creek above West Fork

Date: July 7, 1978

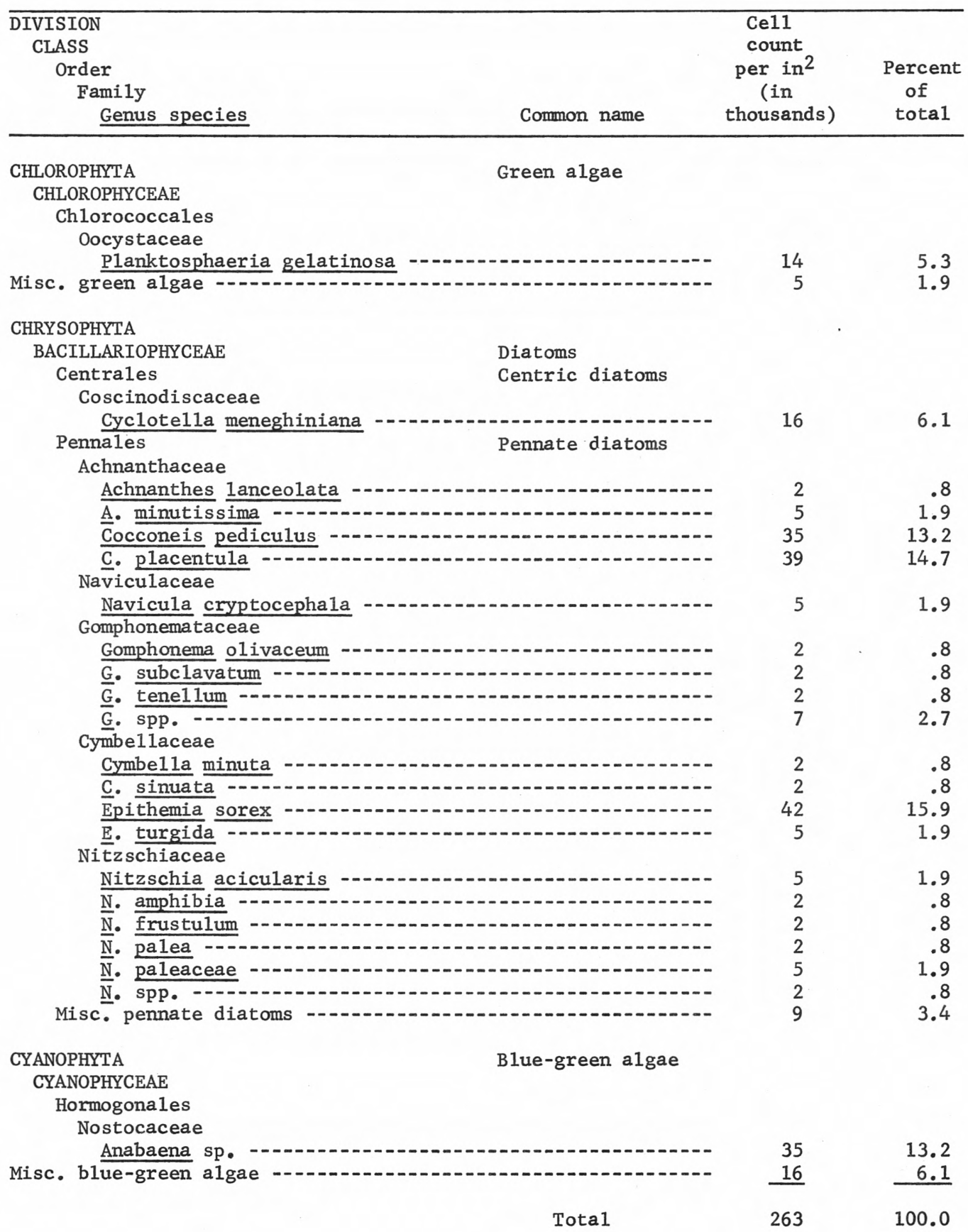


Site number and name: 2 - West Fork Evans Creek

Date: August 11, 1977

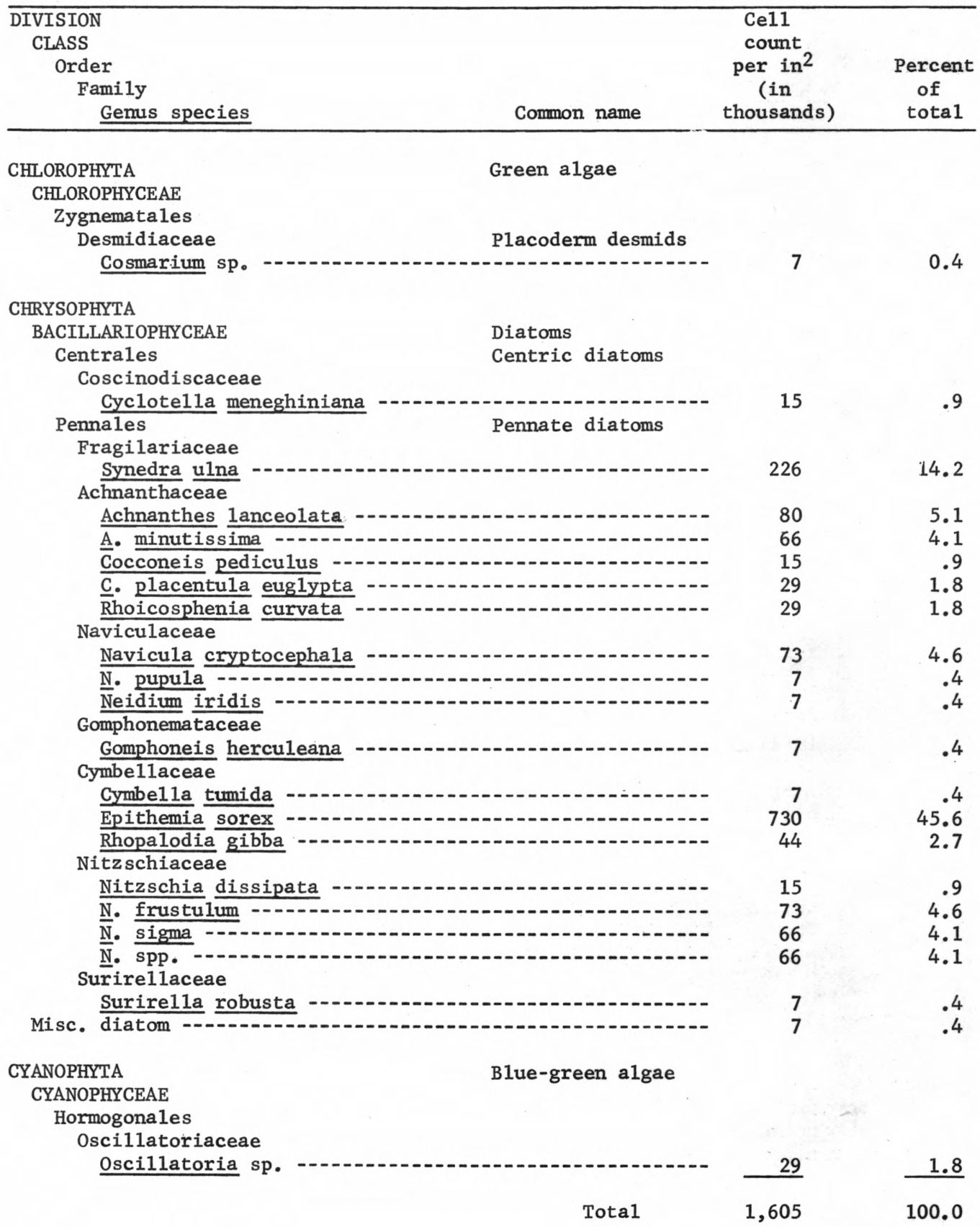


Site number and name: 2 - West Fork Evans Creek

Date: September 8, 1977

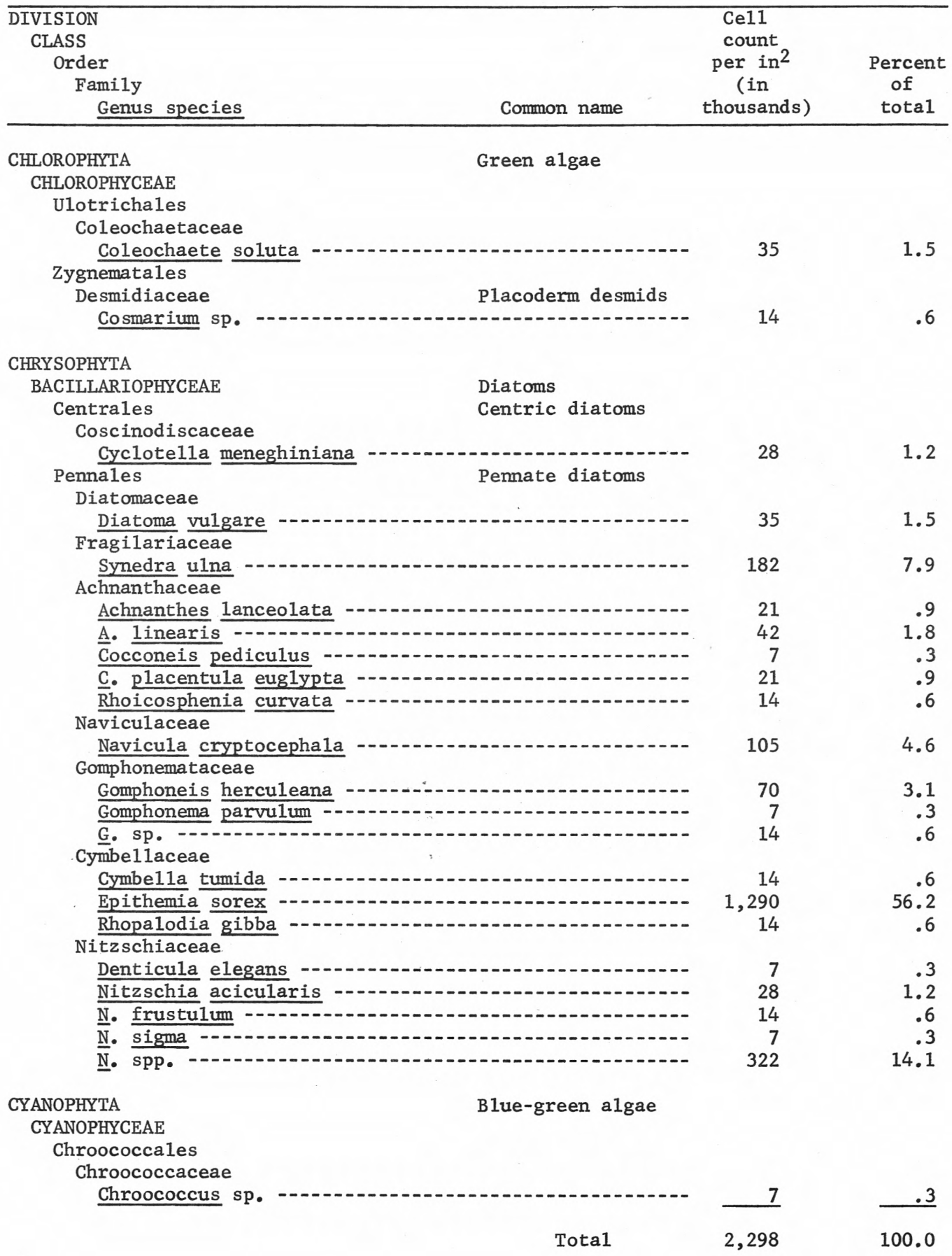


Table 5.--Taxa and numbers of periphyton--Continued

Site number and name: 2 - West Fork Evans Creek

Date:

July 7, 1978

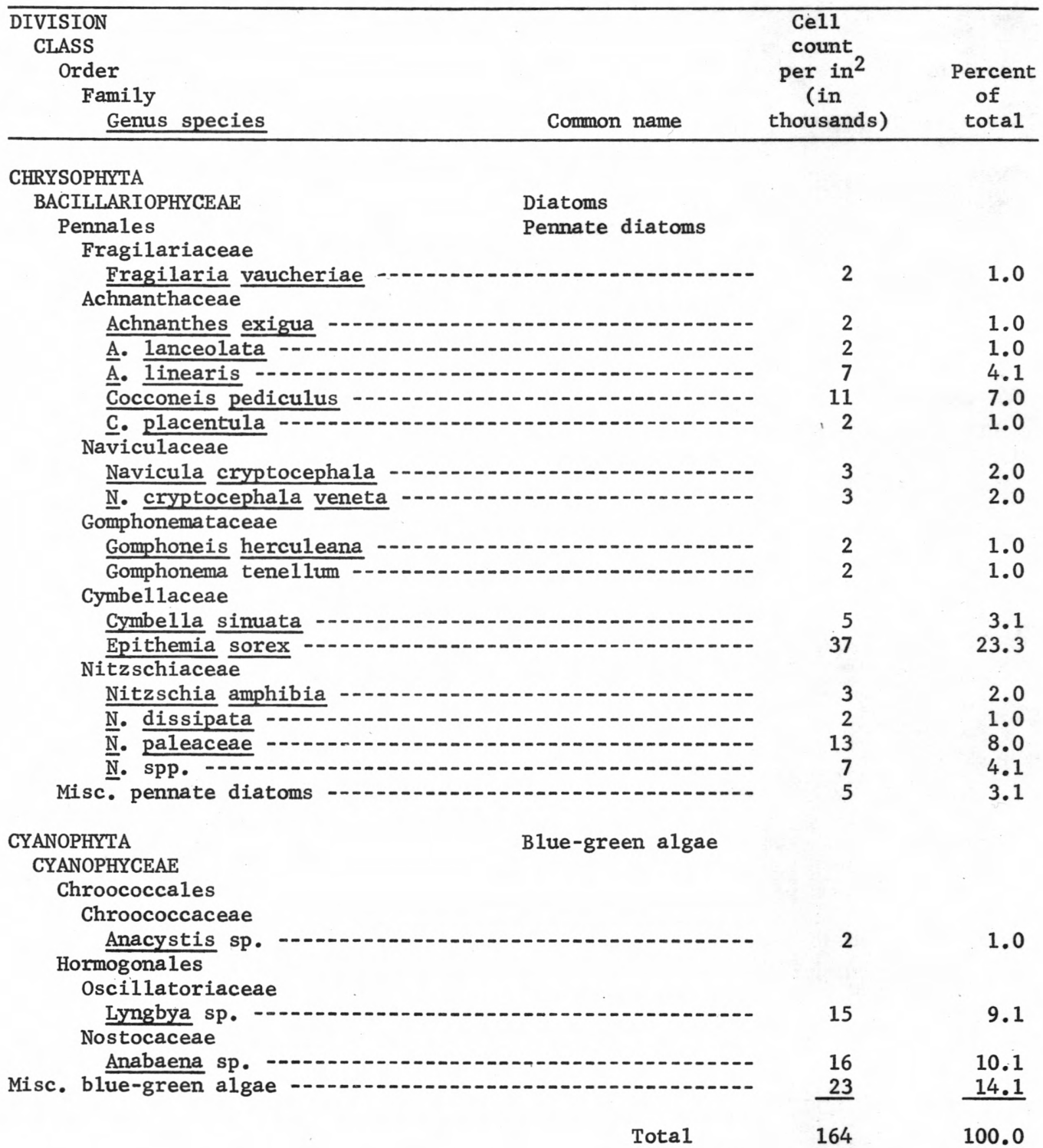


Site number and name: 3 - Evans Creek at Minthorne Road

Date:

September 7, 1977

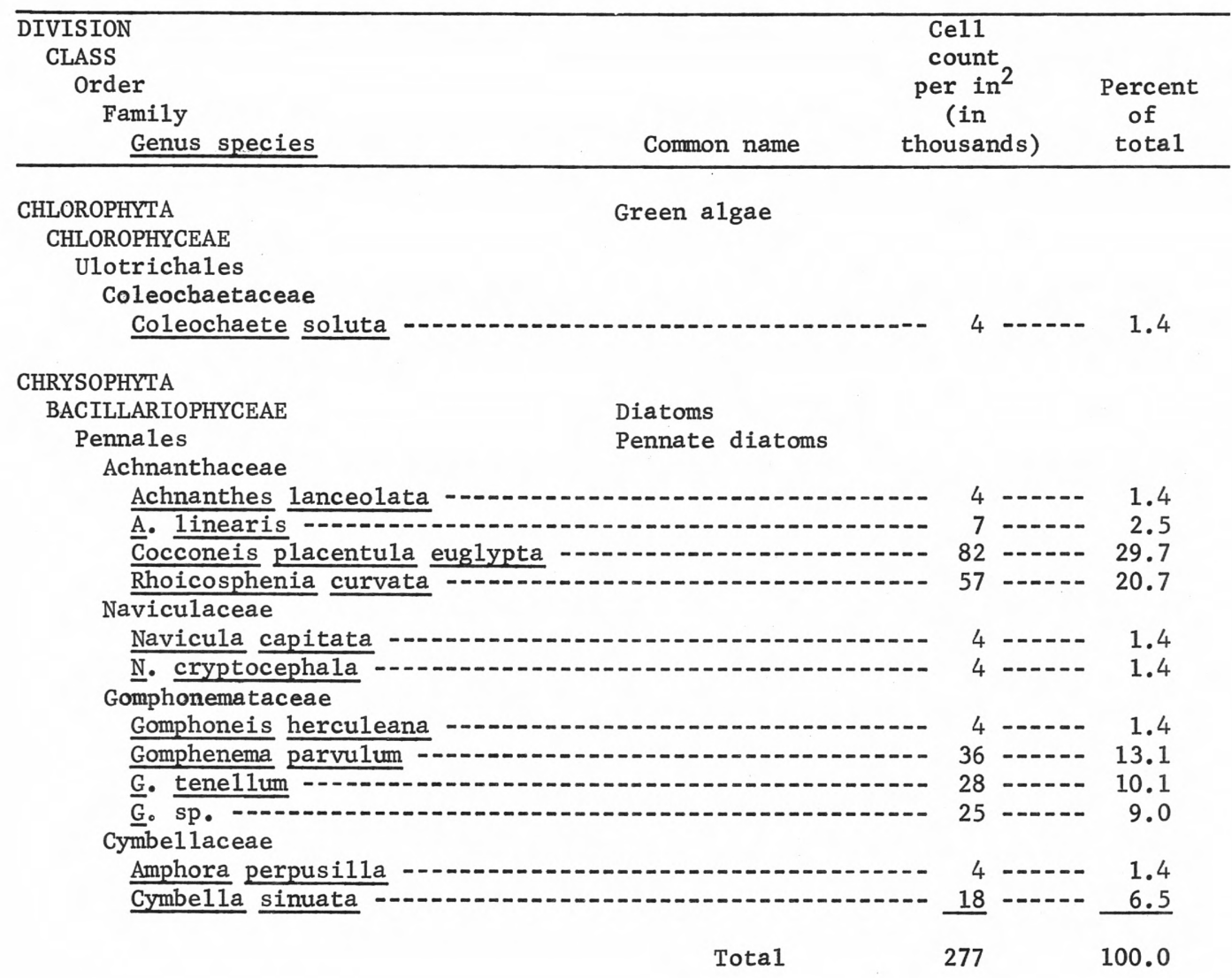


Site number and name: 3 - Evans Creek at Minthorne Road

Date: July 5, 1978

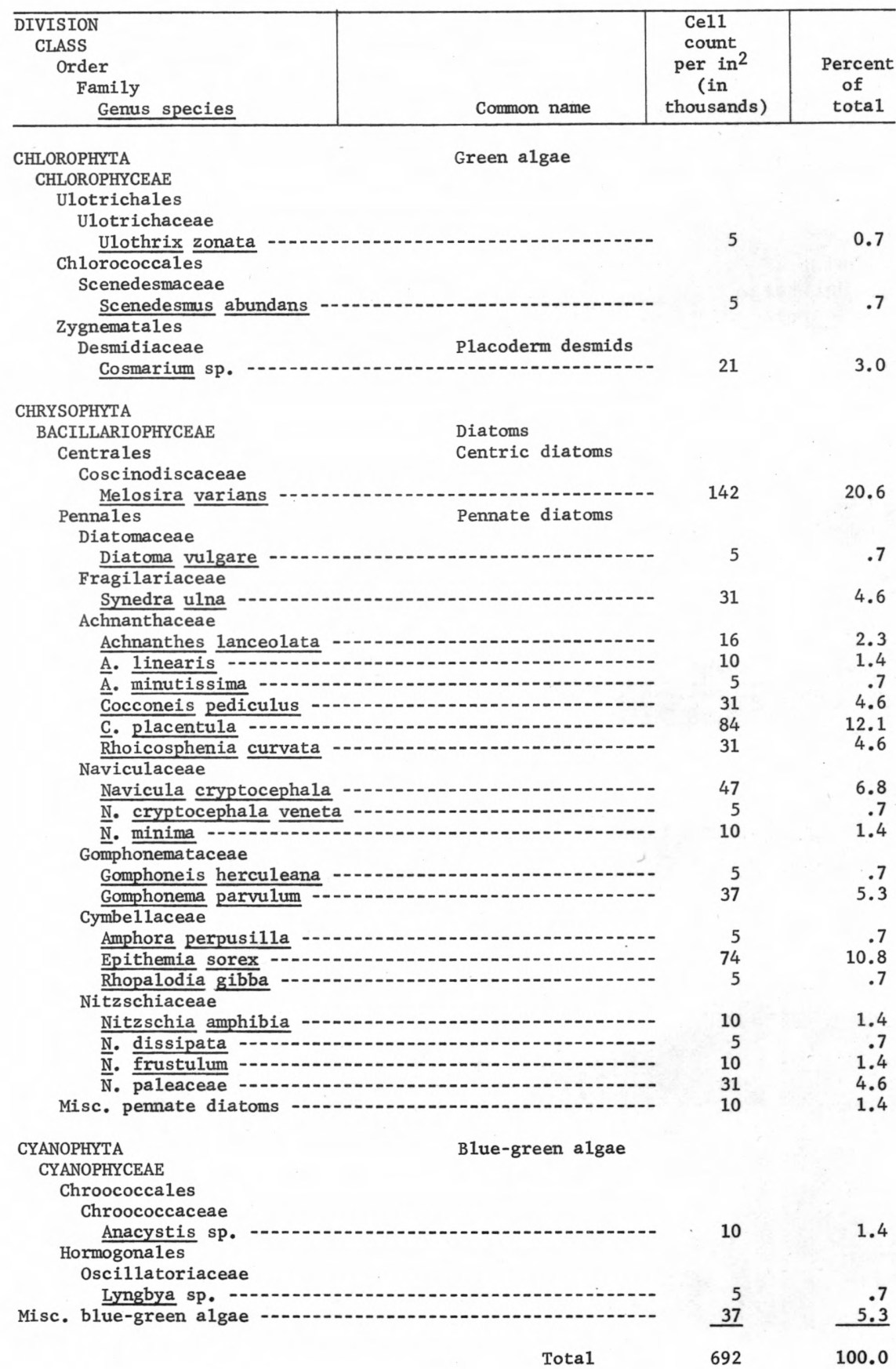


Site number and name: 4 - Lost Creek, Molalla River basin

Date: July 21, 1977

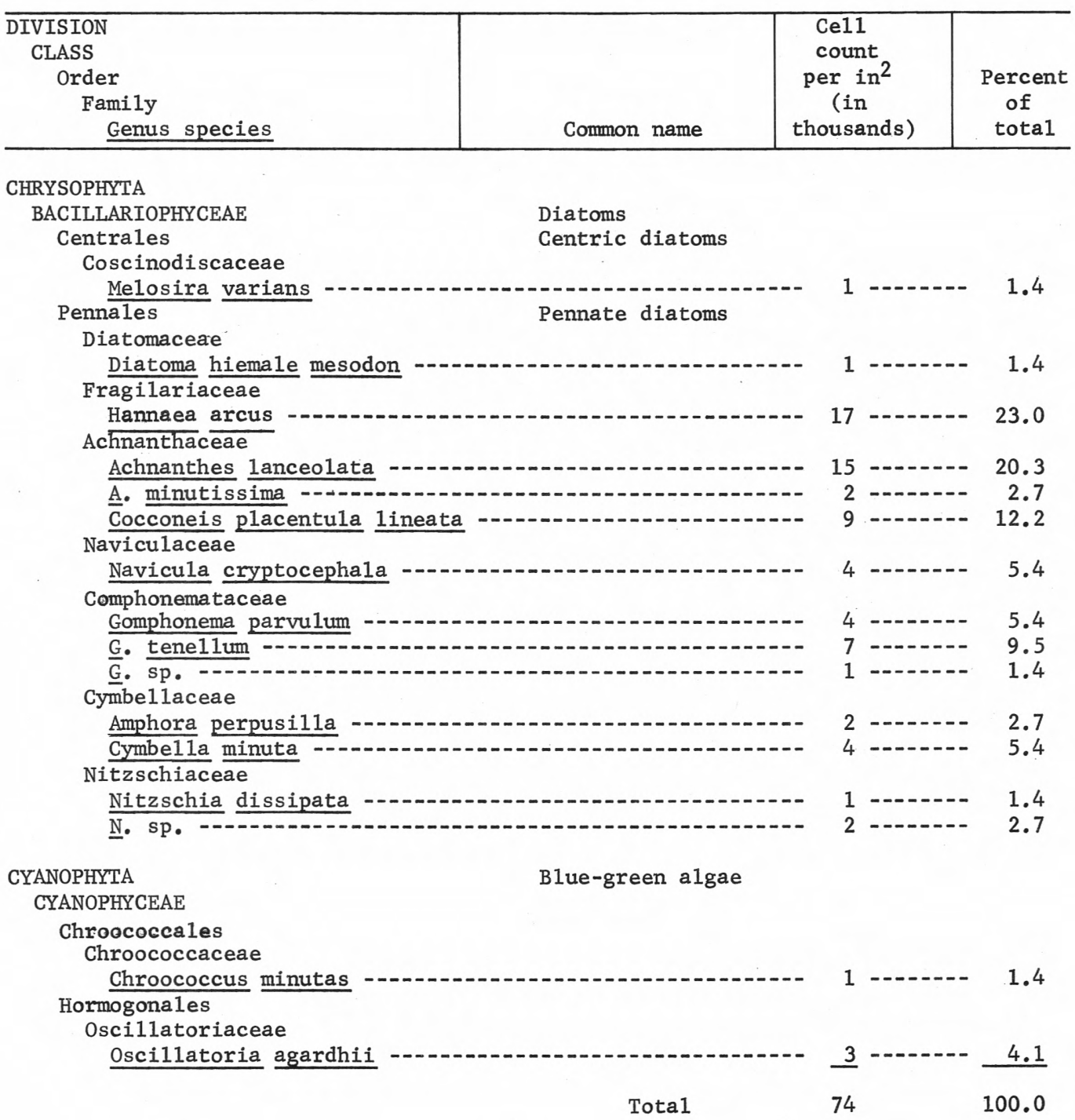


Site number and name: 5 - Dead Horse Canyon Creek, Mola1la River basin

Date: September 15, 1977

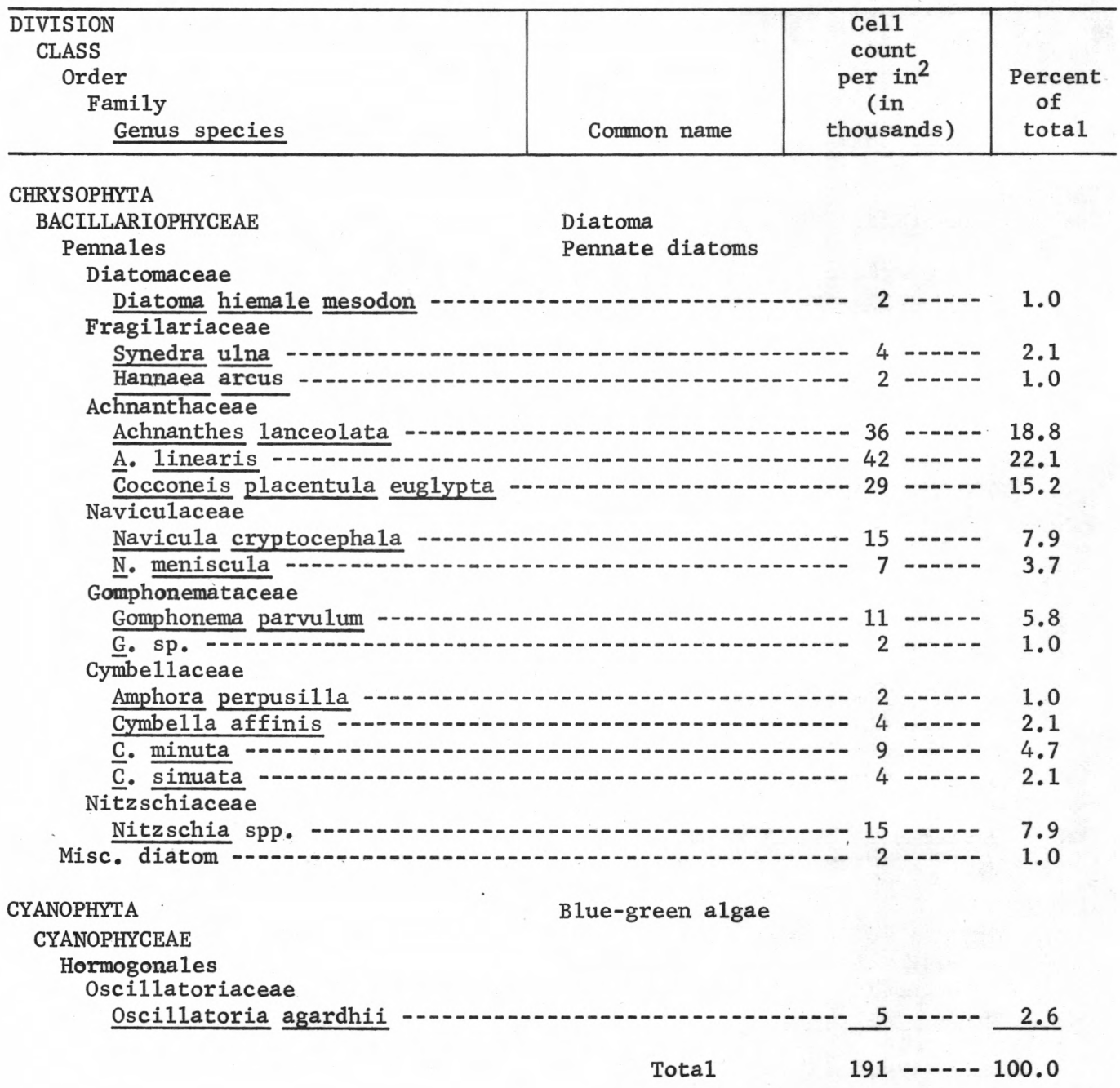


Table 5.--Taxa and numbers of periphyton--Continued

Site number and name: 6 - Dorn Creek, Mola1la River basin

Date: September 14, 1977

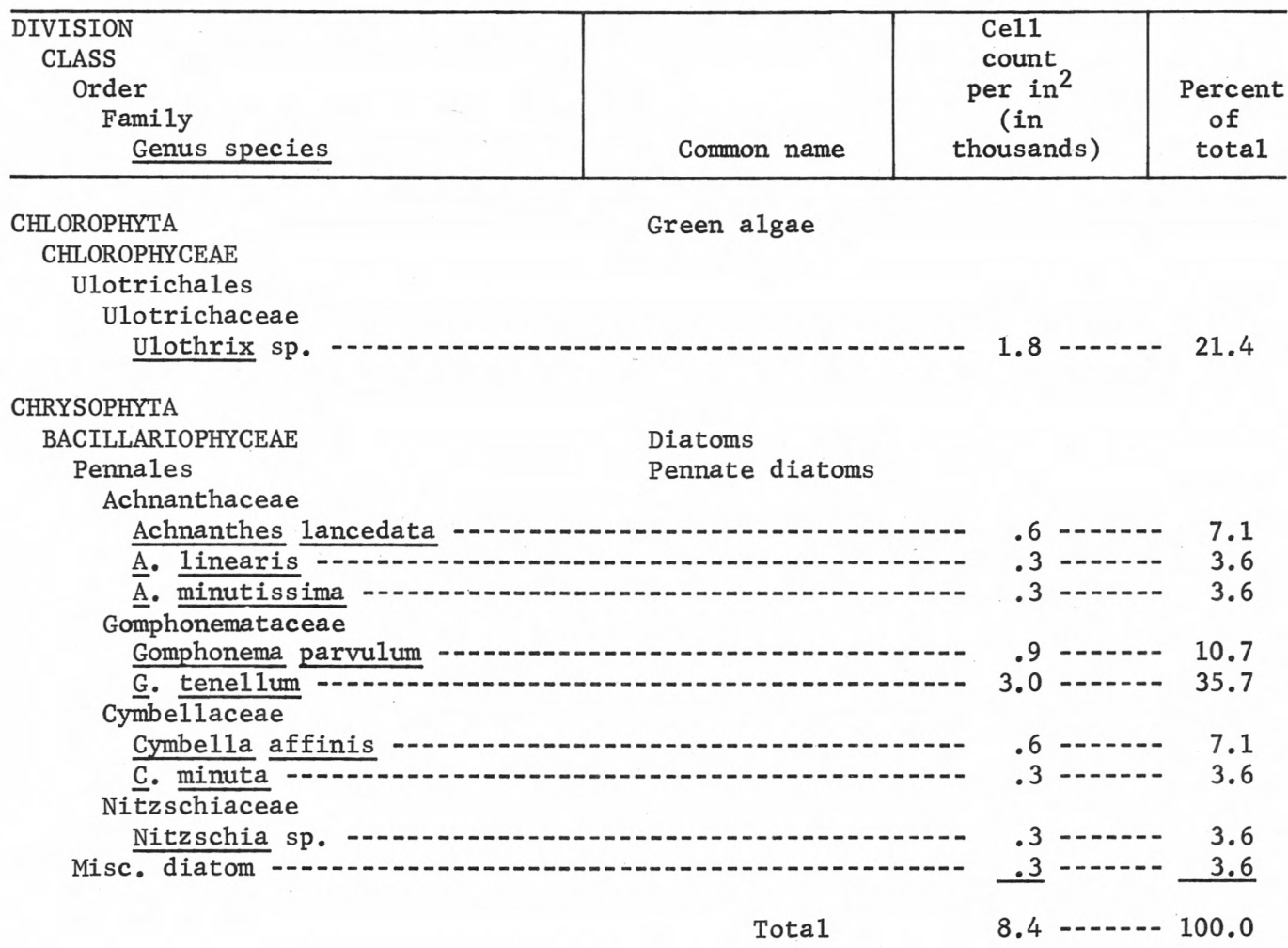


Site number and name: 7 - Milk Creek, Molalla River basin

Date: September 13, 1977

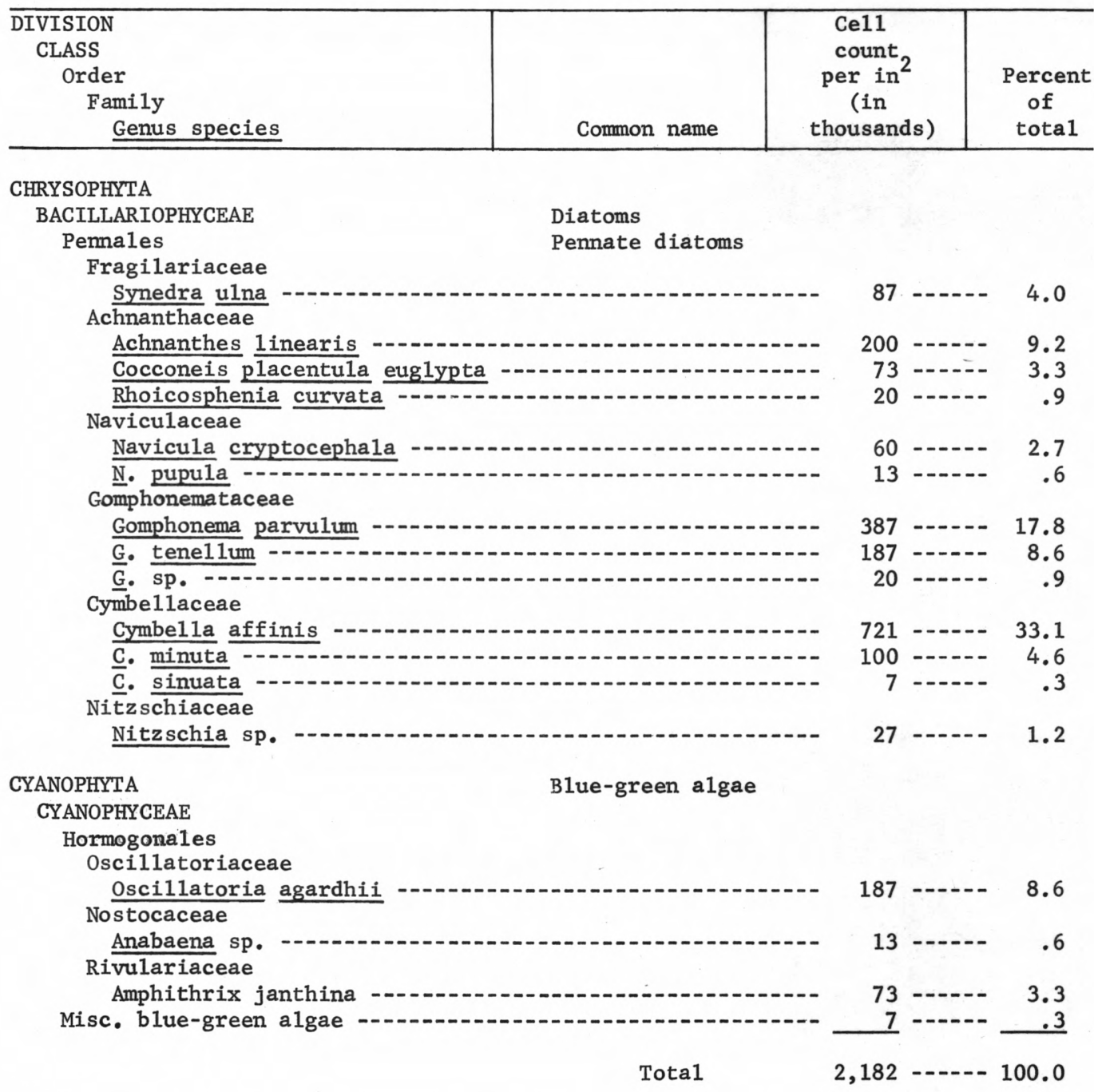


Site number and name: 8 - Wolf Creek, Siuslaw River basin

Date: October 4, 1977

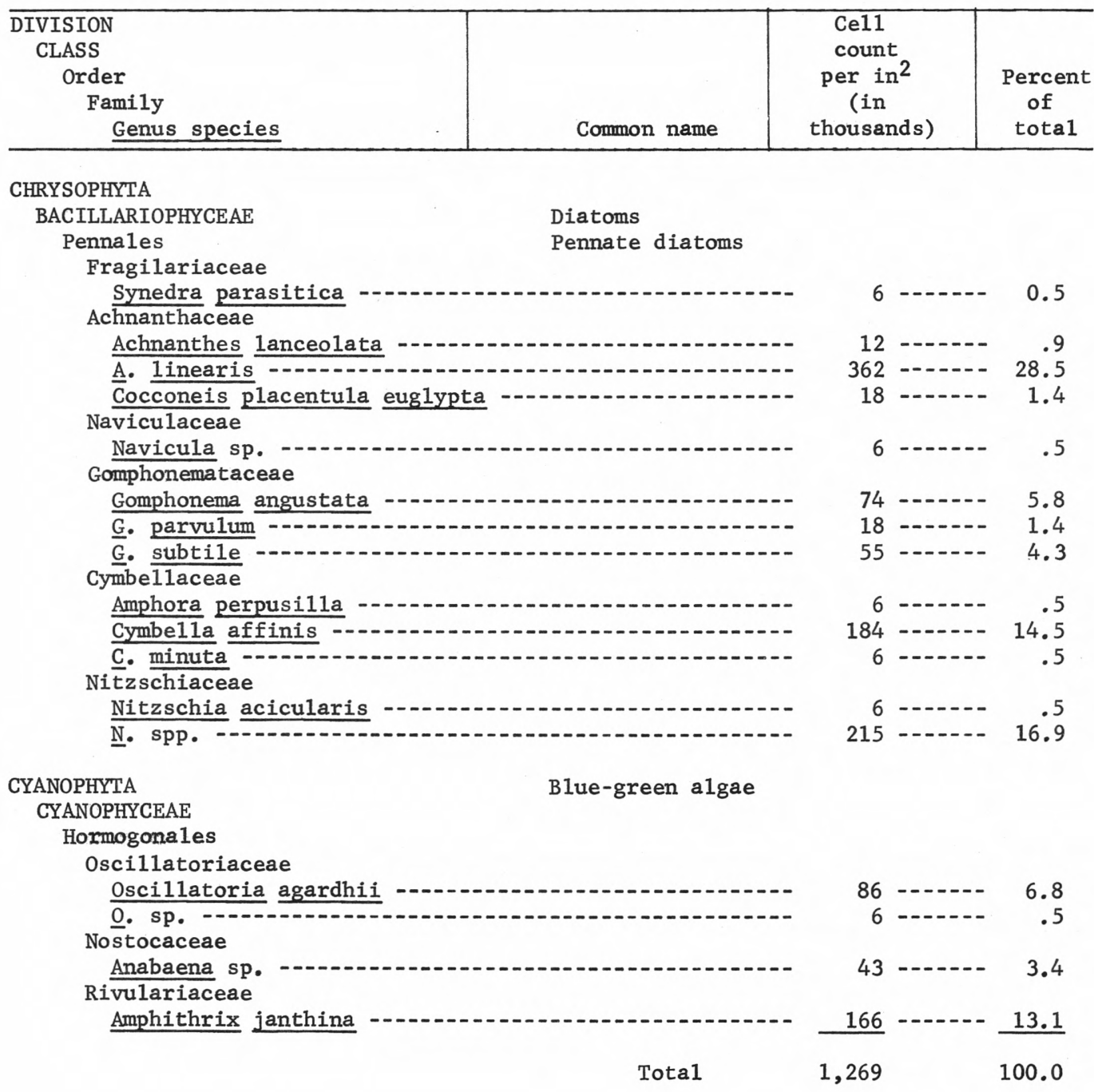


Site number and name: 9 - Knowles Creek, Siuslaw River basin

Date: October 4, 1977

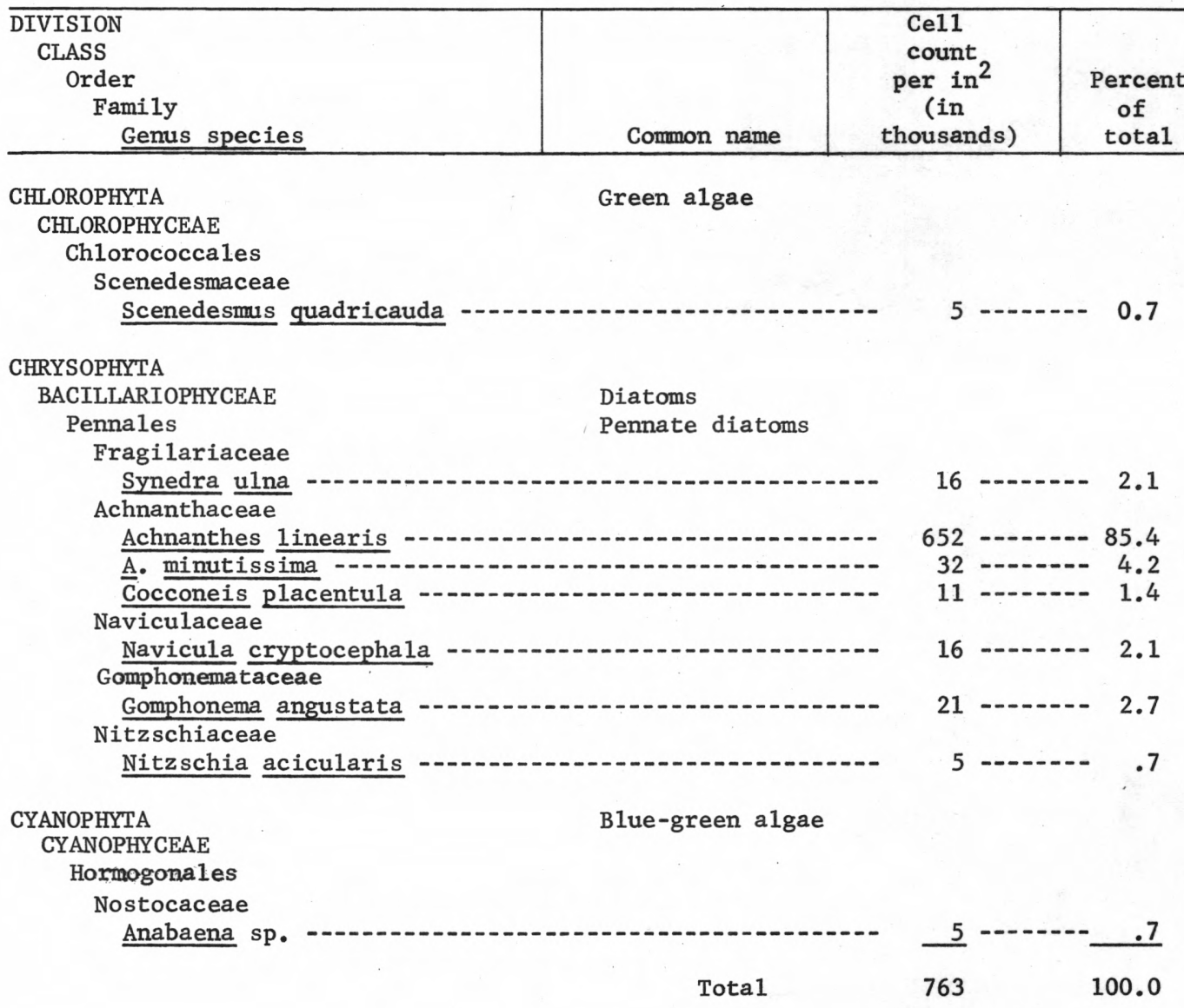


Table 5.--Taxa and numbers of periphyton--Continued

Site number and name: 10-Cedar Creek, Siuslaw River basin

Date: August 10, 1977

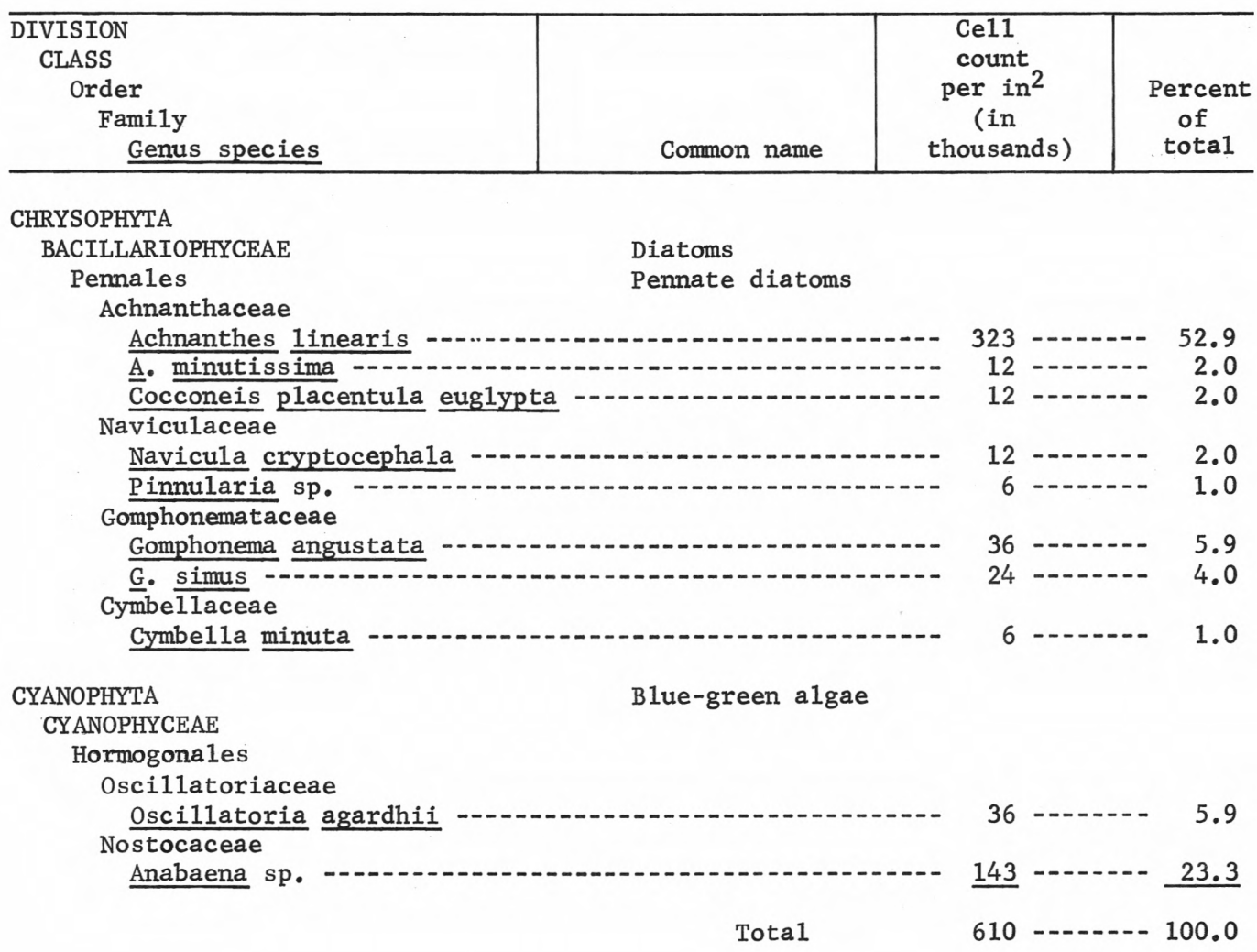


Table 5.--Taxa and numbers of periphyton--Continued

Site number and name: 10 -Cedar Creek, Siuslaw River basin

Date: October 5, 1977

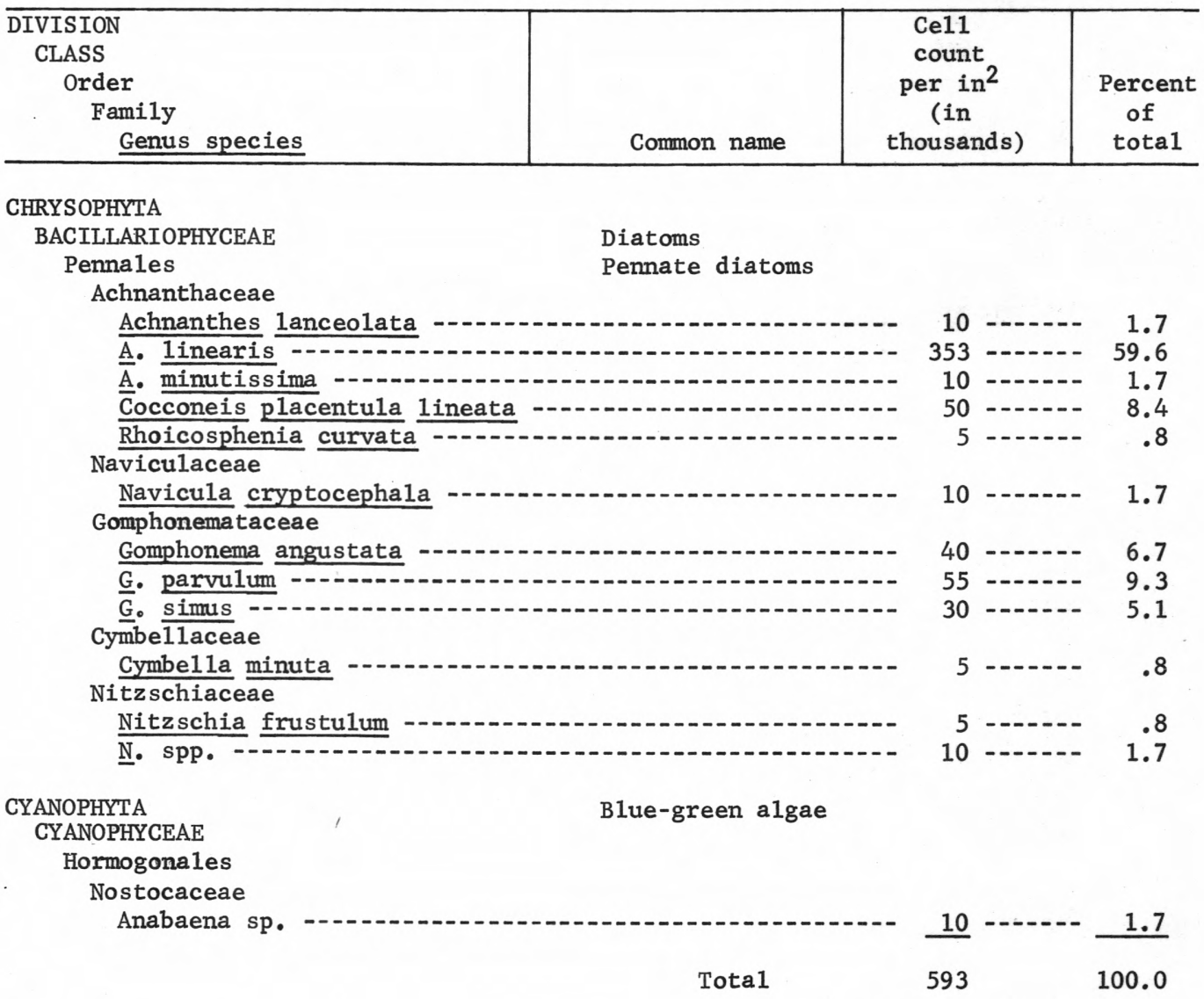


Site number and name: 11-Bob Creek, Siuslaw Basin

Date: August 9, 1977

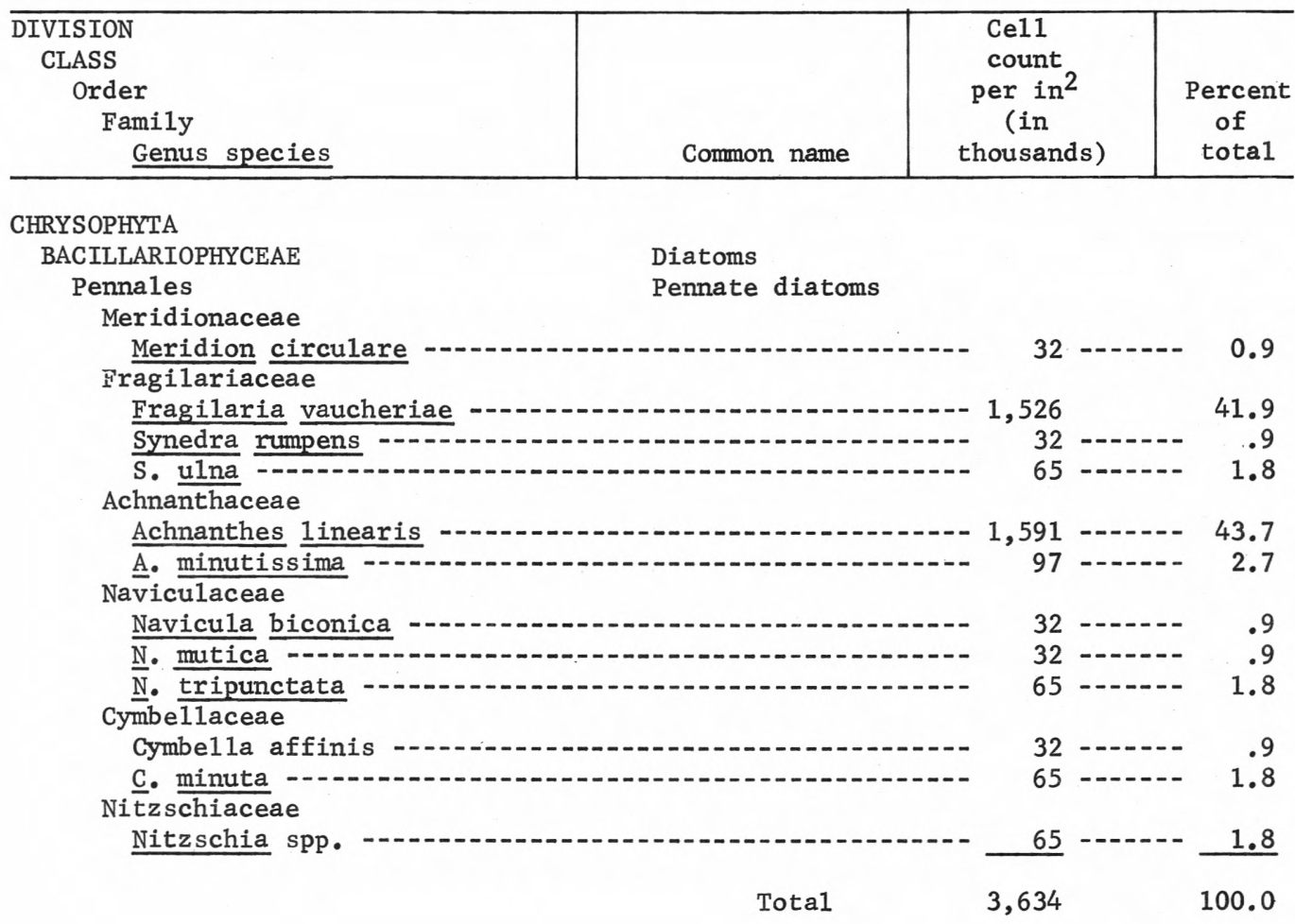


Site number and name: 11 - Bob Creek, Siuslaw Basin

Date: October 5, 1977

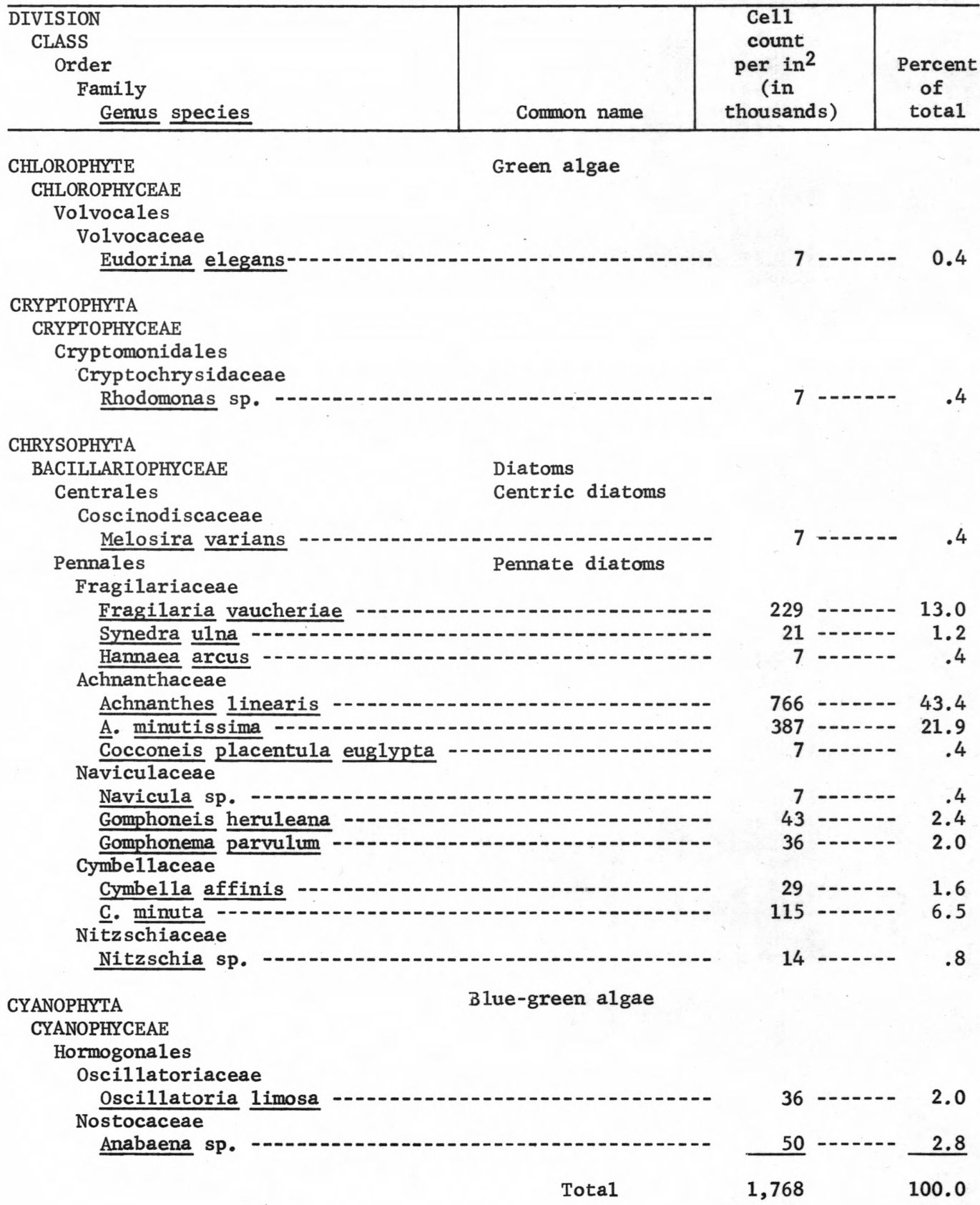


Site number and name: 12 - South Fork John Day River above Forest Service boundary

Date: September 15,1978

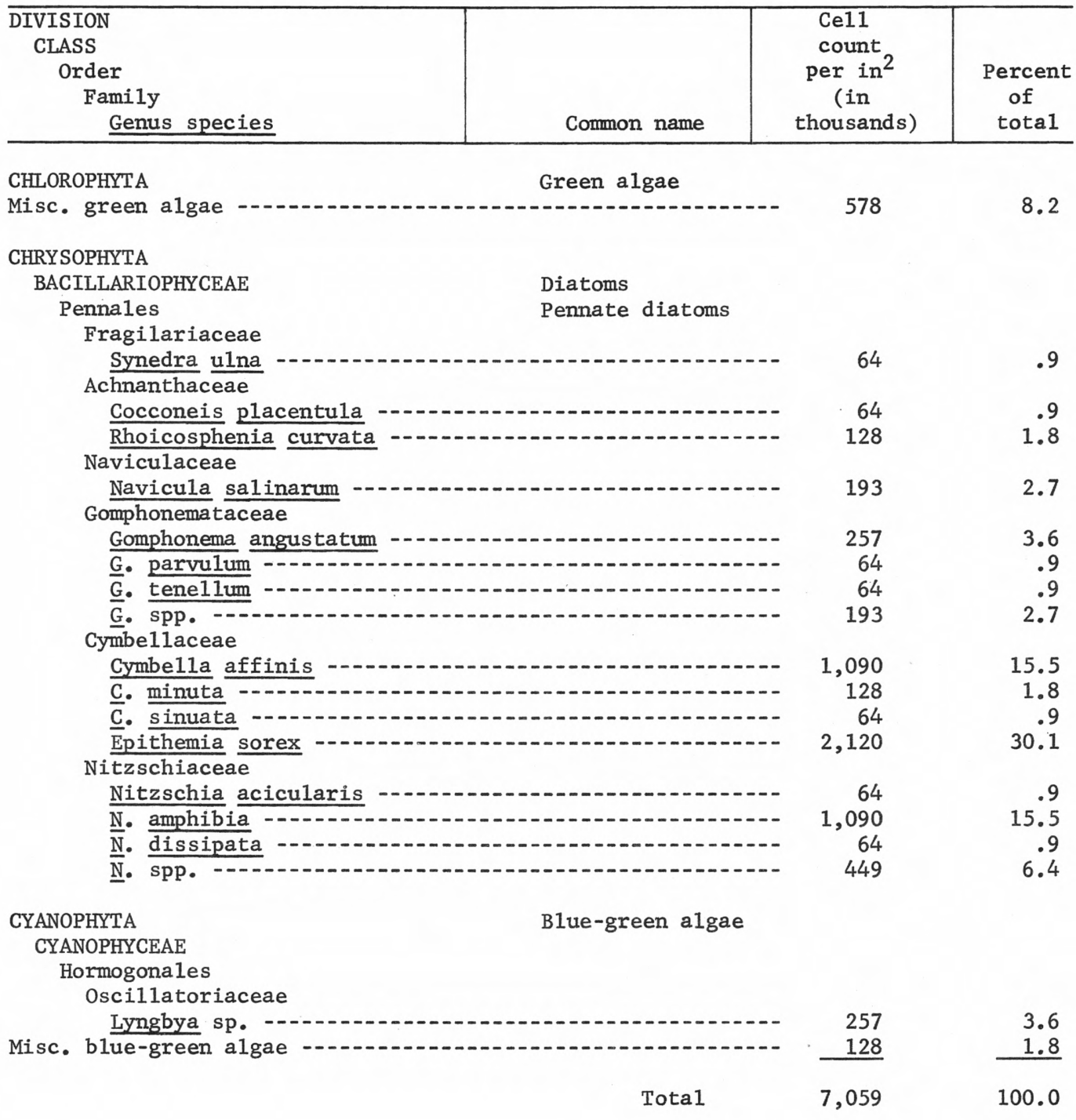


Site number and name: 13 - South Fork John Day River above Little Pine Creek

Date: September 15,1978

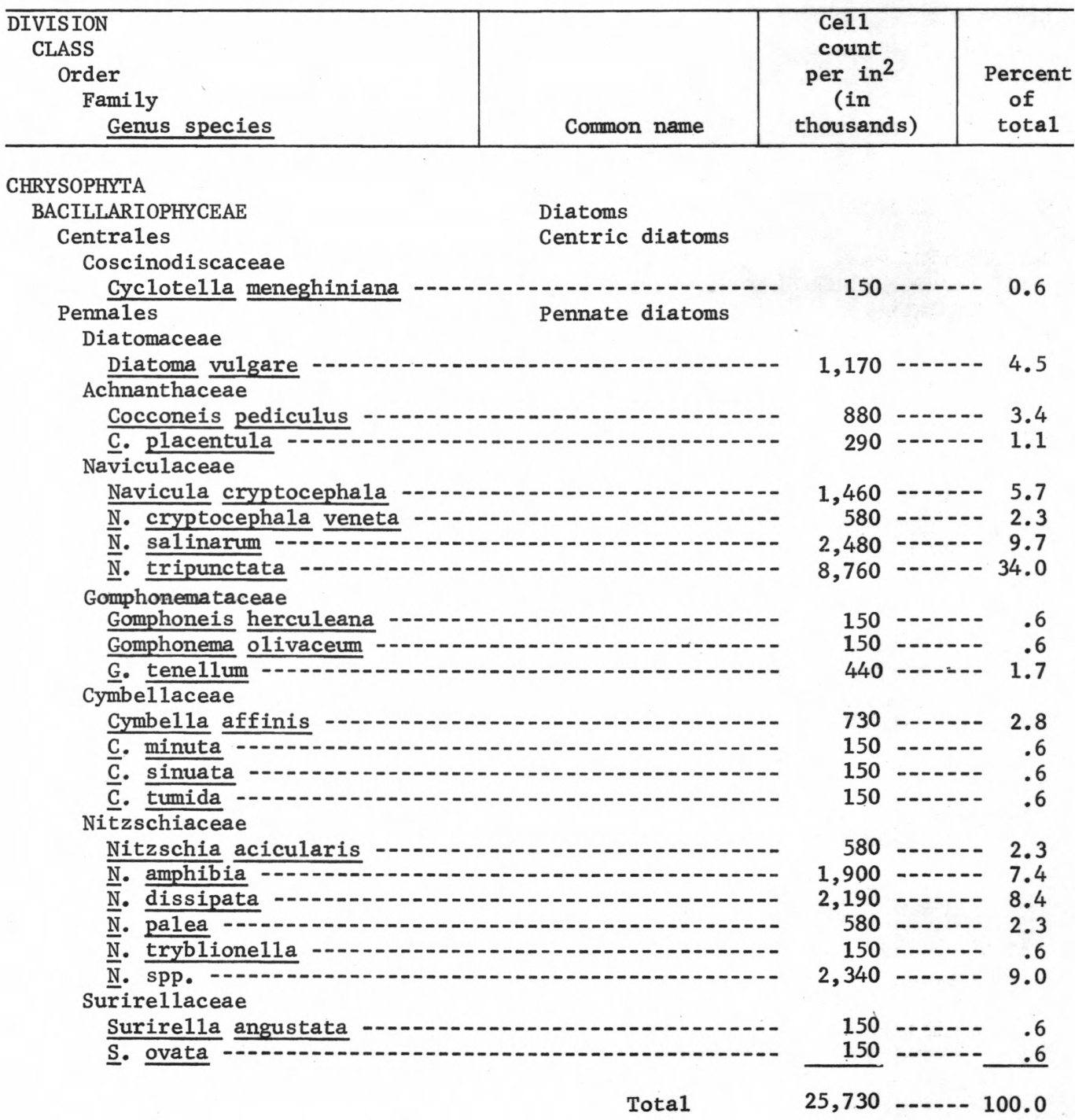


Site number and name: 14 - Black Canyon Creek, South Fork John Day River Basin

Date: September 14,1978

\begin{tabular}{l|c|c|c}
\hline DIVISION & & $\begin{array}{c}\text { Cel1 } \\
\text { count } \\
\text { pLASS }\end{array}$ & \\
$\begin{array}{l}\text { Order } \\
\text { Family } \\
\text { Genus species }\end{array}$ & (in & $\begin{array}{c}\text { Percent } \\
\text { of } \\
\text { tot }\end{array}$ \\
\hline
\end{tabular}

CHLOROPHYTA

CHLOROPHYCEAE

Zygnematales

Desmidiaceae Placoderm desmids

Cosmarium sp.

Green algae

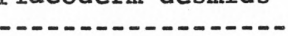

CHRYSOPHYTA

BACILLARIOPHYCEAE Diatoms

Pennales Pennate diatoms

Fragilariaceae

Synedra mazamaensis -

S. rumpens -1.-1-1.-1

Achnanthaceae

Achnanthes $\frac{\text { exigua }}{\text { Anceolata }}$ 1ance

A. 1inearis -

A. minutissima -

Cocconeis placentula -

Rhoicosphenia curvata

Naviculaceae

Navicula cryptocephala veneta.

N. tripunctata

Gomphonemataceae

Gomphoneis herculeana

Gomphonema subclavatum -...

G. tenellum -

Cymbe11aceae

Cymbella sinuata

C. tumida

Epithemia sorex

Nitzschiaceae

Nitzschia amphibia

N. dissipata -

N. recta -

$\overline{\mathrm{N}}$. spp.

Misc. pennate diatoms

CYANOPHYTA

CYANOPHYCEAE

Hormogonales

Oscillatoriaceae

Lyngbya sp.

Oscillatoria sp. Nostocaceae

Anabaena circinalis

Blue-green algae

\begin{tabular}{rr}
145 & 7.9 \\
81 & 4.5 \\
32 & 1.8 \\
113 & 6.3 \\
\hline 1,806 & 100.0
\end{tabular}


Site number and name: 15 - South Fork John Day River at Dayville

Date:

August 12, 1977

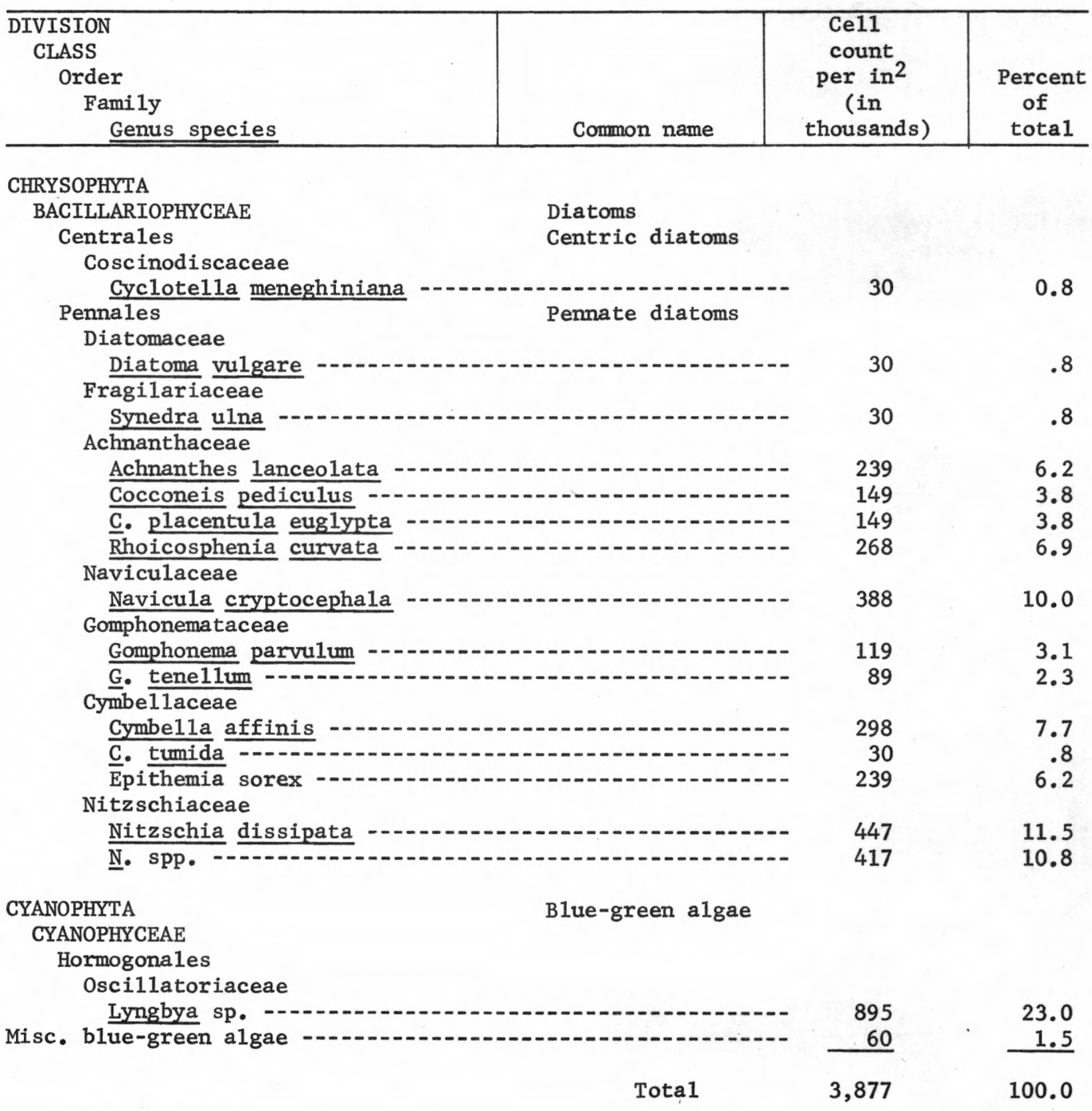


Table 5.--Taxa and numbers of periphyton--Continued

Site number and name: 15 - South Fork John Day River at Dayville

Date: September 7, 1977

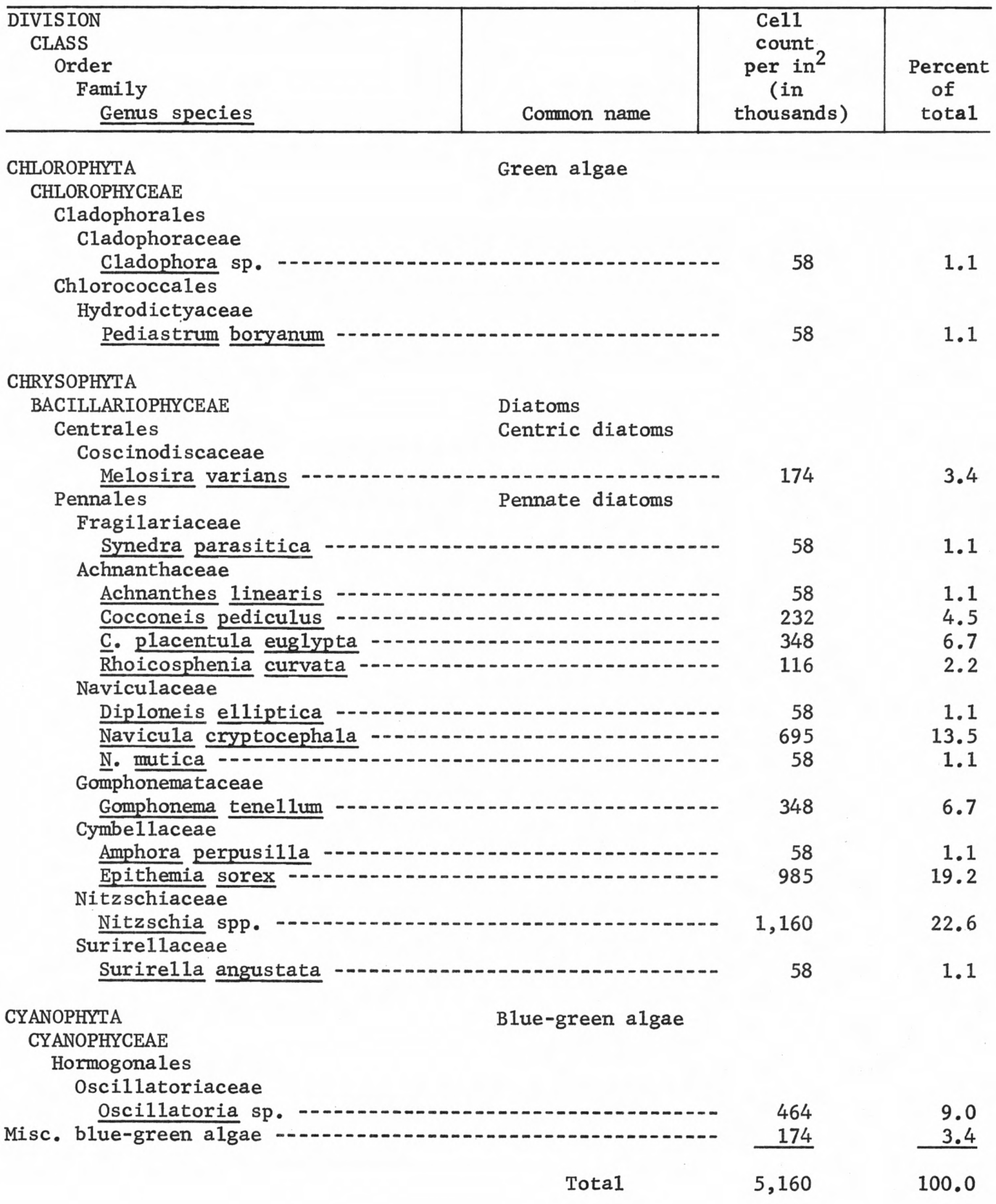


Site number and name: 15 - South Fork John Day River at Dayville

Date: September 13,1978

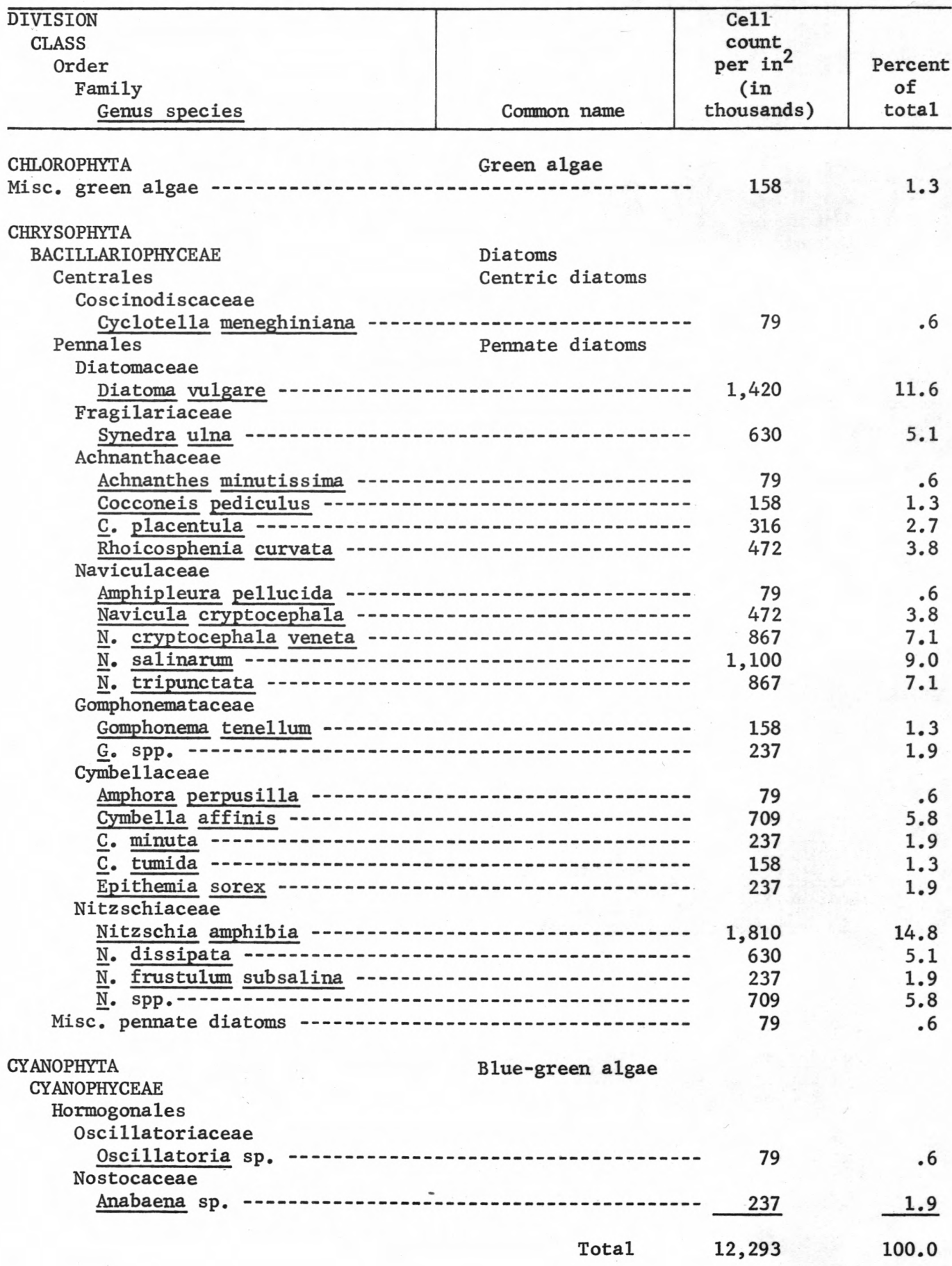


Site number and name: 16 - Pearson Creek, Umatilla River basin

Date: September 20, 1978

\begin{tabular}{|c|c|c|c|}
\hline $\begin{array}{l}\text { DIVISION } \\
\text { CLASS } \\
\text { Order } \\
\text { Family } \\
\text { Genus species }\end{array}$ & Common name & $\begin{array}{l}\text { Ce11 } \\
\text { count } \\
\text { per in } 2 \\
\text { (in } \\
\text { thousands) }\end{array}$ & $\begin{array}{l}\text { Percent } \\
\text { of } \\
\text { total }\end{array}$ \\
\hline
\end{tabular}

CHLOROPHYTA

Green algae

CHLOROPHYCEAE

Ulotrichales

Ulotrichaceae

Ulothrix zonata

Zygnematales

Desmidiaceae

Placoderm desmids

Closterium sp.

Placoderm desmids

CHRYSOPHYTA

BACILLARIOPHYCEAE

Diatoms

Centrales

Centric diatoms

Coscinodiscaceae

Melosira varians

Pennales

Pennate diatoms

Meridionaceae

Meridion circulare

Fragilariaceae

Synedra mazamaensis --0-1-0.

S. ulna =-1..-

Achnanthaceae

Achnanthes 1inearis -

Cocconeis placentula

Rhoicosphenia curvata

Naviculaceae

Amphipleua pellucida -

Navicula cryptocephala -

N. cryptocephala veneta

$\overline{\mathrm{N}}$. Salinarum -

N. tripunctata

Gomphonemataceae

Gomphoneis herculeana -

Gomphonema spp. -

Cymbellaceae

Cymbe1la affinis -

C. minuta -1

C. sinuata -

Epithemia turgida -.

Nitzschiaceae

Nitzschia amphibia -

N. dissipata -

Misc。 pennate diatoms

Blue-green algae

CYANOPHYTA

CYANOPHYCEAE

Hormogonales

Nostocaceae

Anabaena sp.

Misc. blue-green algae -

Tota1

43 
Site number and name: 17. - East Fork Birch Creek, Umatilla River basin

Date: September 21,1978

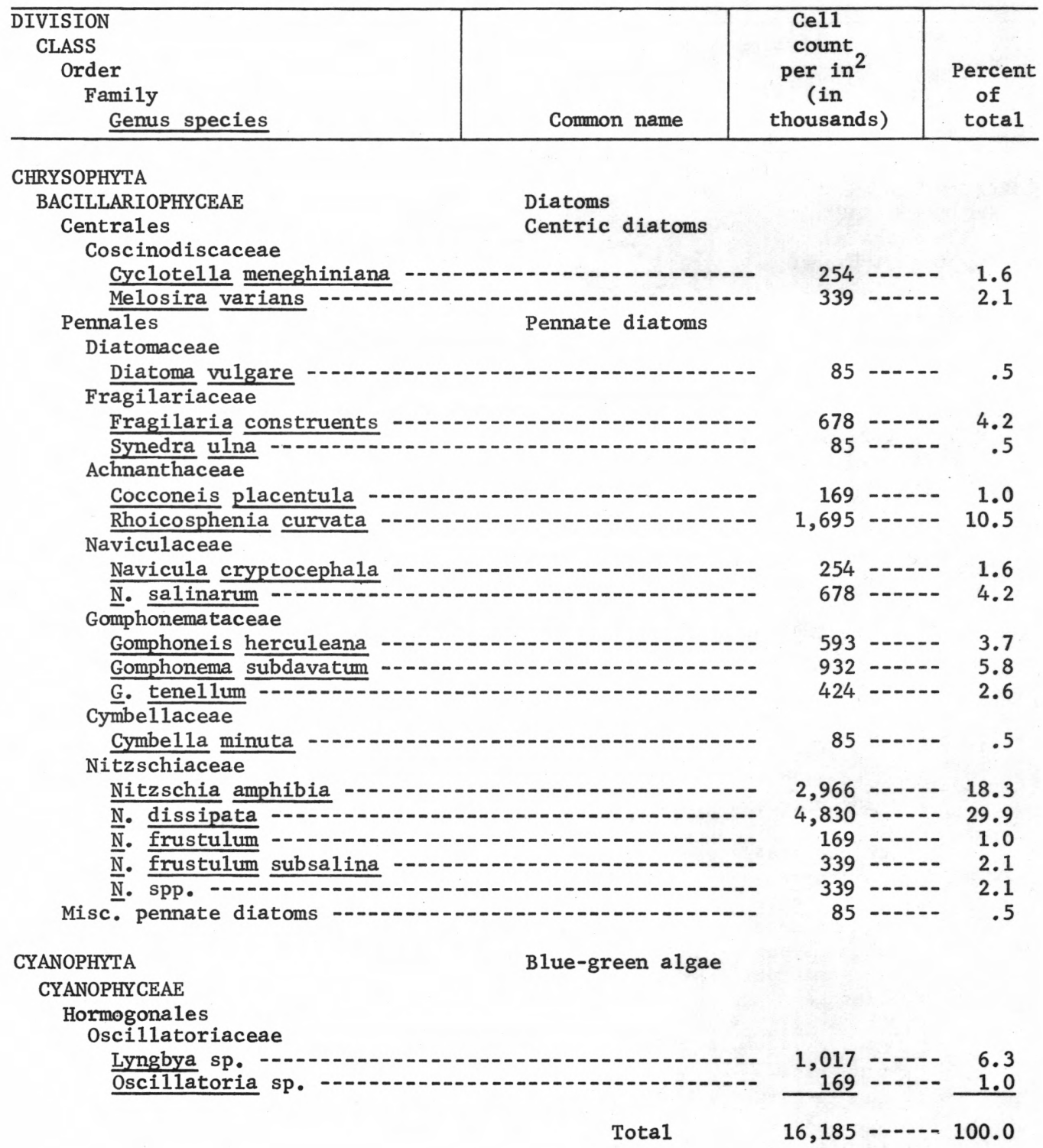


Site number and name: 18 -Wildhorse Creek, Umatilla River basin

Date: September 22, 1978

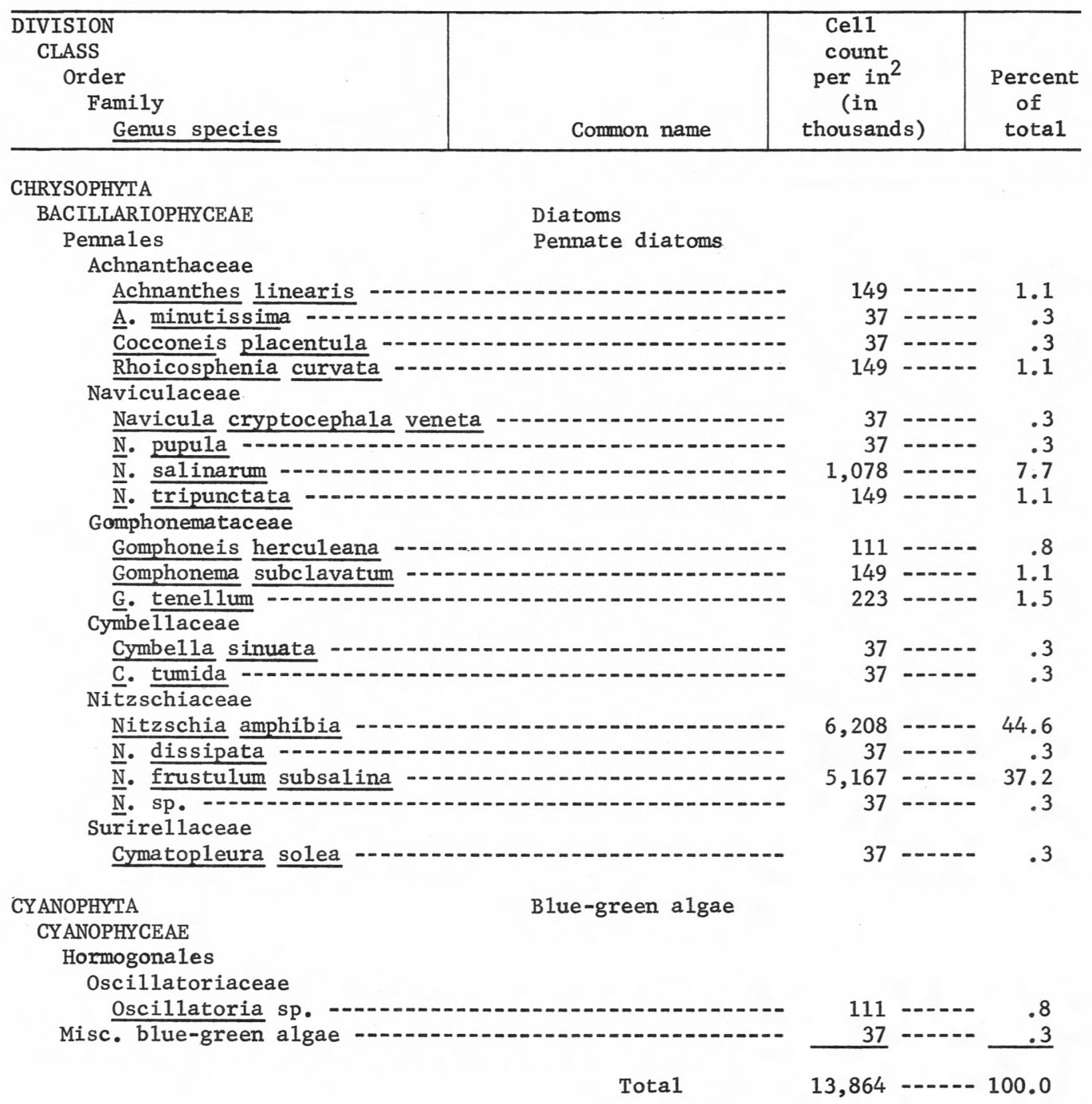


Site number and name: $1 \mathrm{~A}$ - Evans Creek above West Fork

Date: August 12, 1977

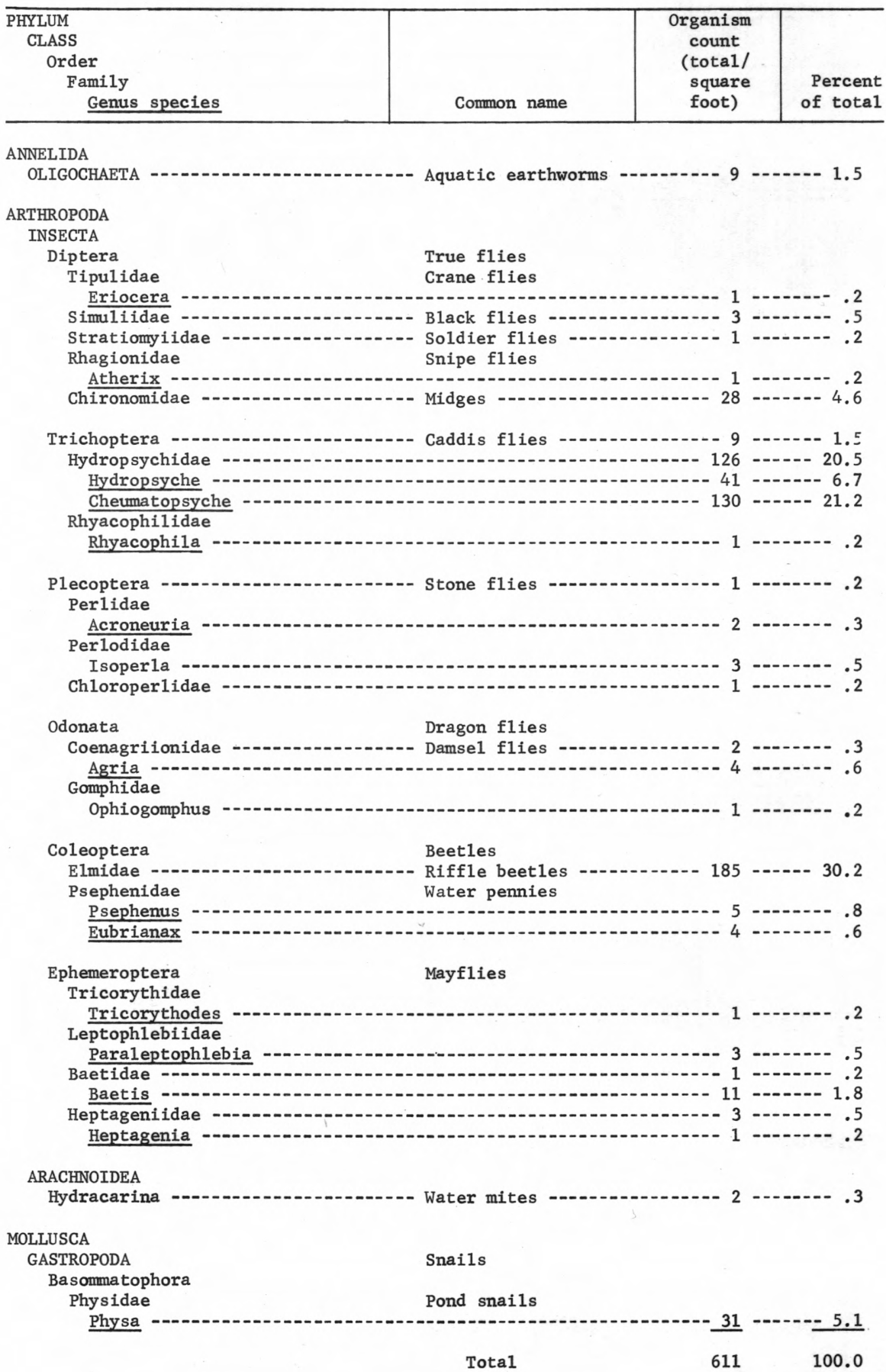


Site number and name: 1B - Evans Creek above West Fork

Date: July 7, 1978

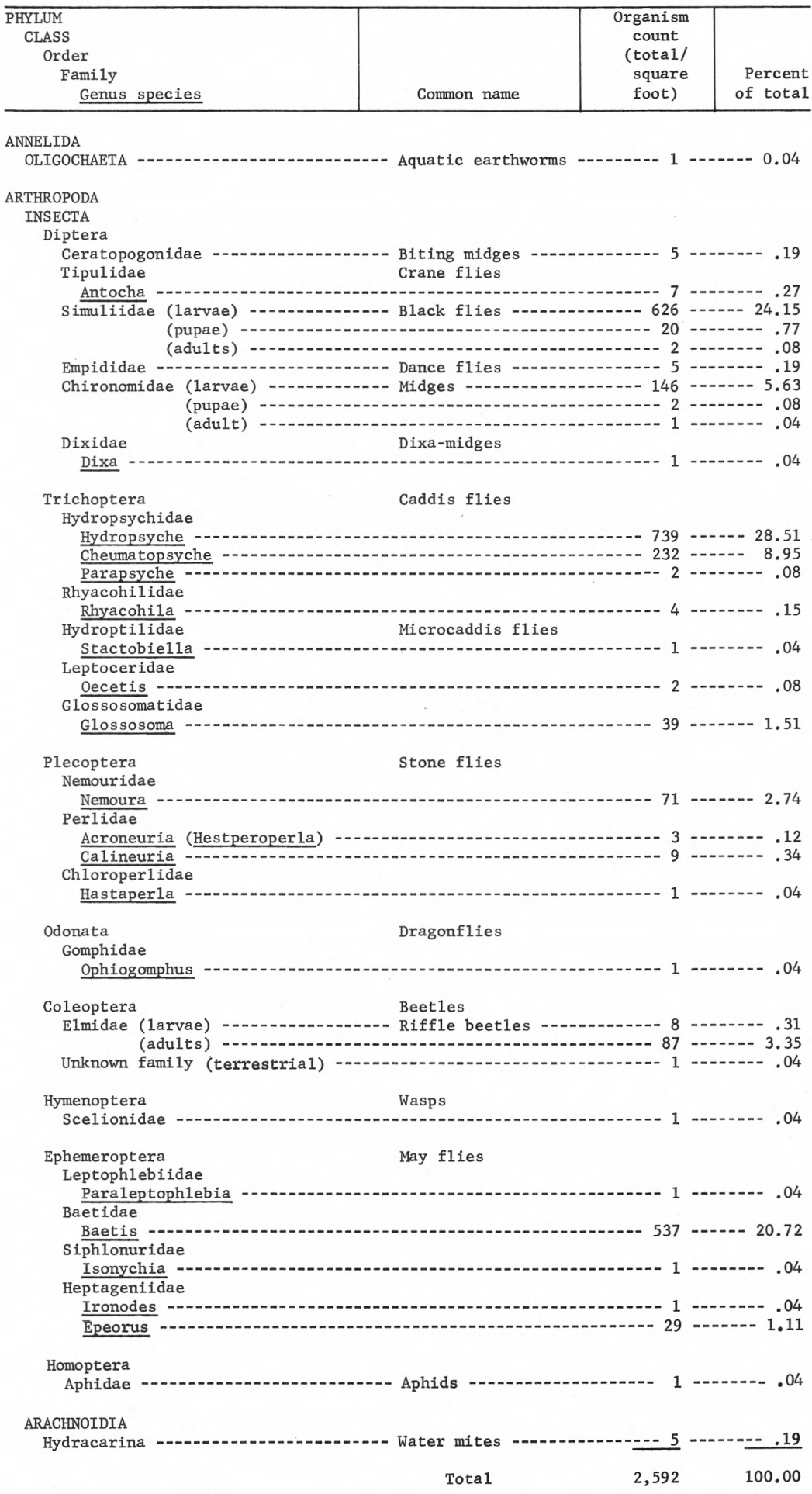


Table 6.--Taxa and numbers of benthic invertebrates--Continued

Site number and name: 2 - West Fork Evans Creek

Date: August 11, 1977

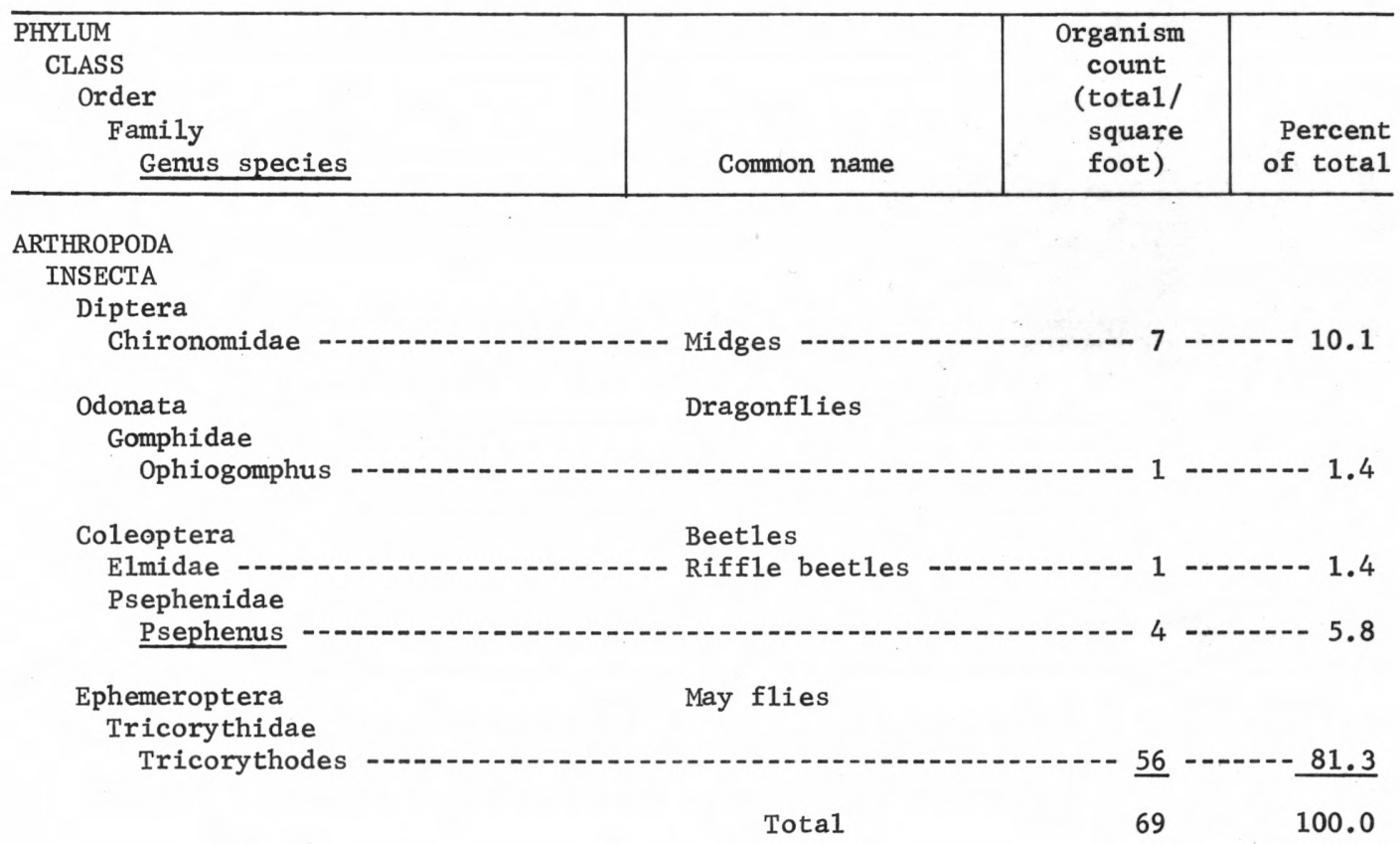


Site number and name 2 - West Fork Evans Creek

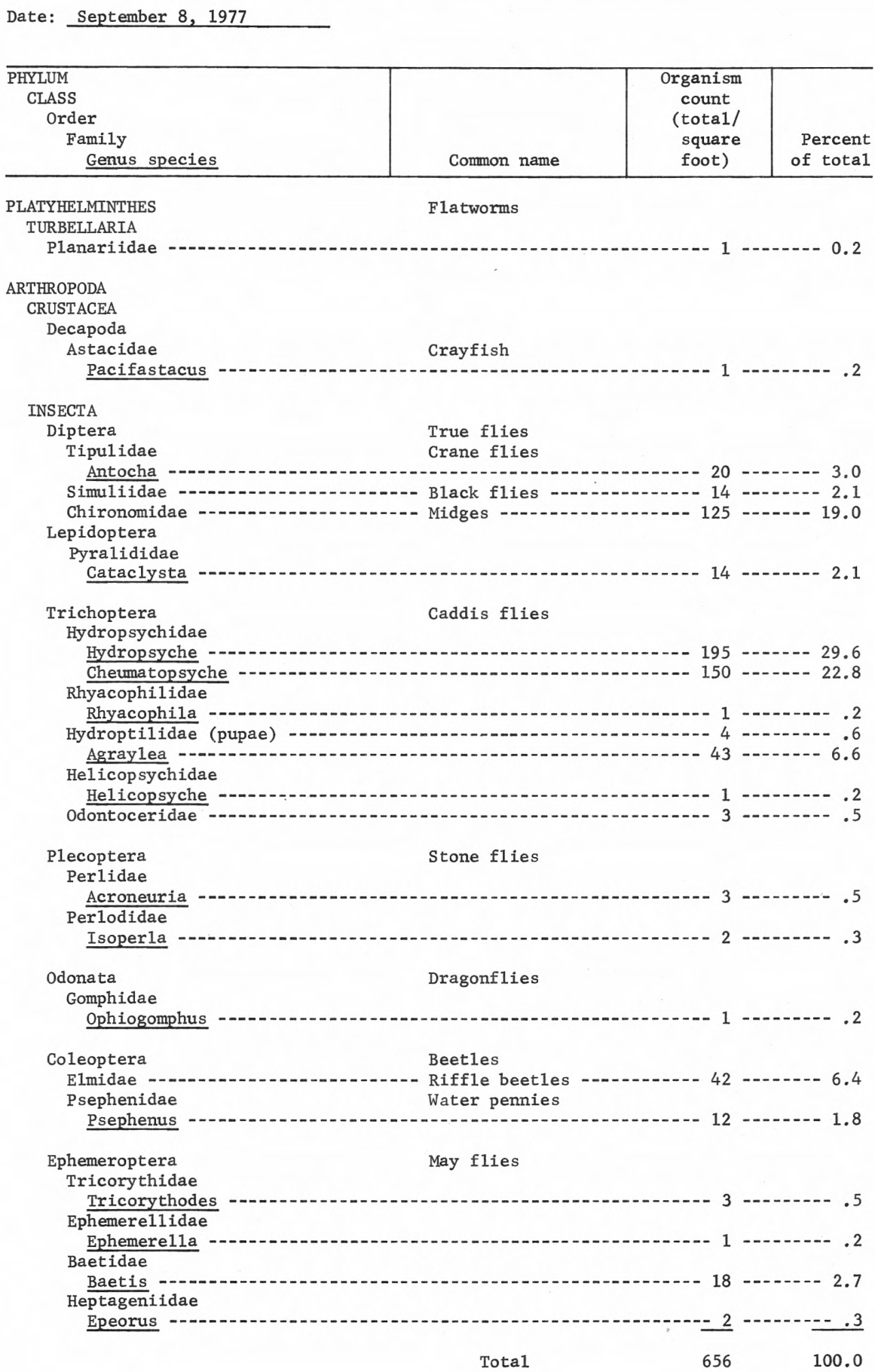




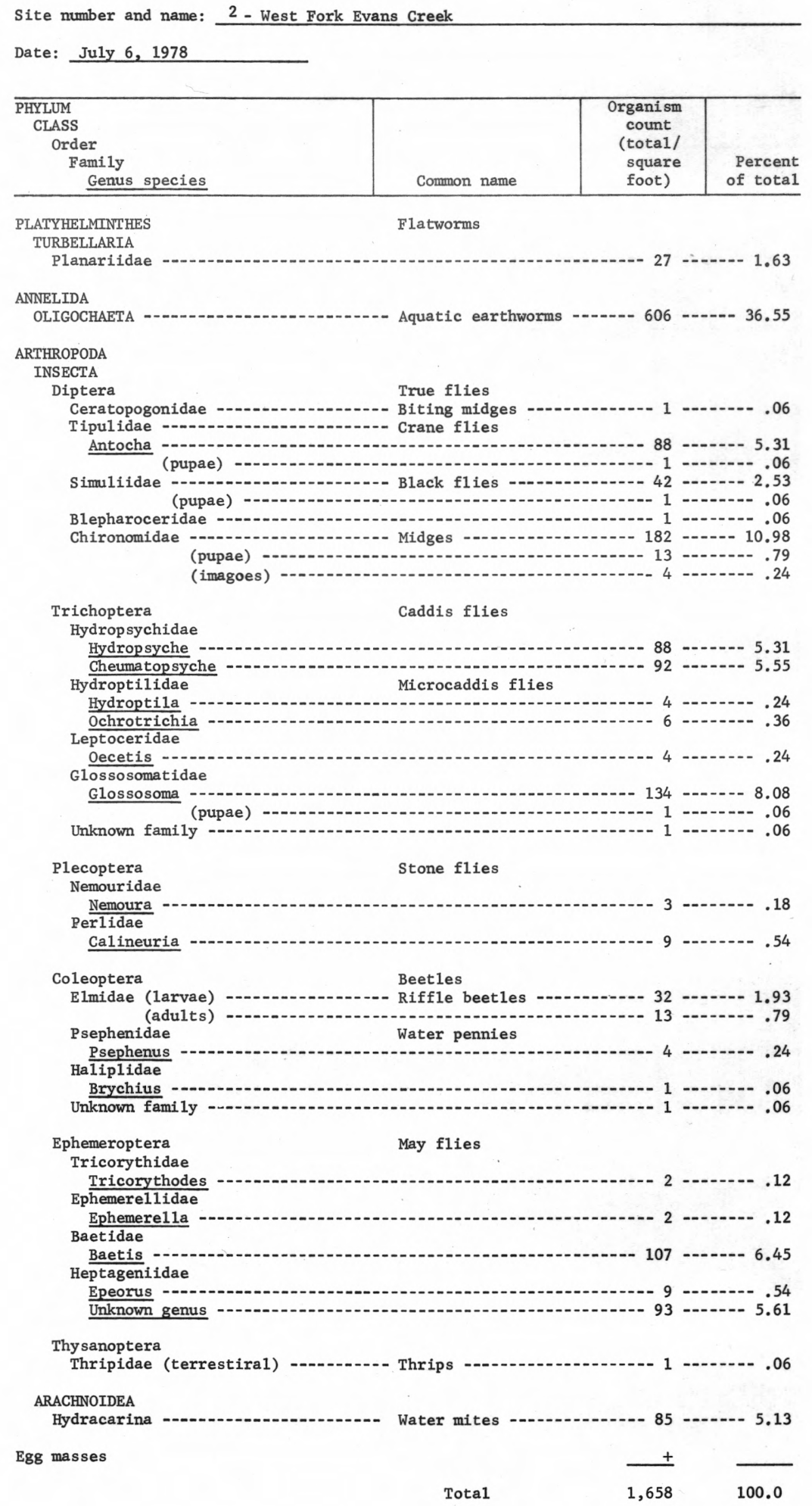


Site number and name: 3 - Evans Creek at Minthorne Road

Date: September 7, 1977

\begin{tabular}{l|c|c|c}
\hline PHYLUM & & Organism & count \\
CLASS & & (tota1/ & square \\
Order & & Percent \\
Family & Common name & foot & of total \\
Genus species & Comon
\end{tabular}

PLATYHELMINTHES Flatworms

TURBELLARIA

Planariidae

ARTHROPODA

INSECTA

Diptera

True flies

Ceratopogonidae - 1 - 1 -

Simuliidae - 3 - 3 -

Chironomidae -....... Midges -

Odonata Dragonflies

Coenagrionidae Damse1 flies

Agria - 7 - 7 - 1.9

Gomphidae -

Hemiptera

True bugs

Corixidae

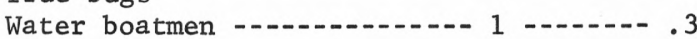

Coleoptera Beetles

Elmidae -

Psephenidae Water pennies

Psephenus - 5 - 5

Ephemeroptera Mayflies

Ephemerellidae

Ephemere11a

Baetidae

Baetis - 4 - 4

MOLLUSCA

GASTROPODA

Mesogastropeda

Pleuroceridae

Goniobasis

Ba sommatophora
Physidae
Pond snails
Physa

Tota1

$\underline{128}-\cdots$

$370 \quad 100.0$ 
Site number and name: 3 - Evans Creek at Minthorne Road

Date: July 6, 1978

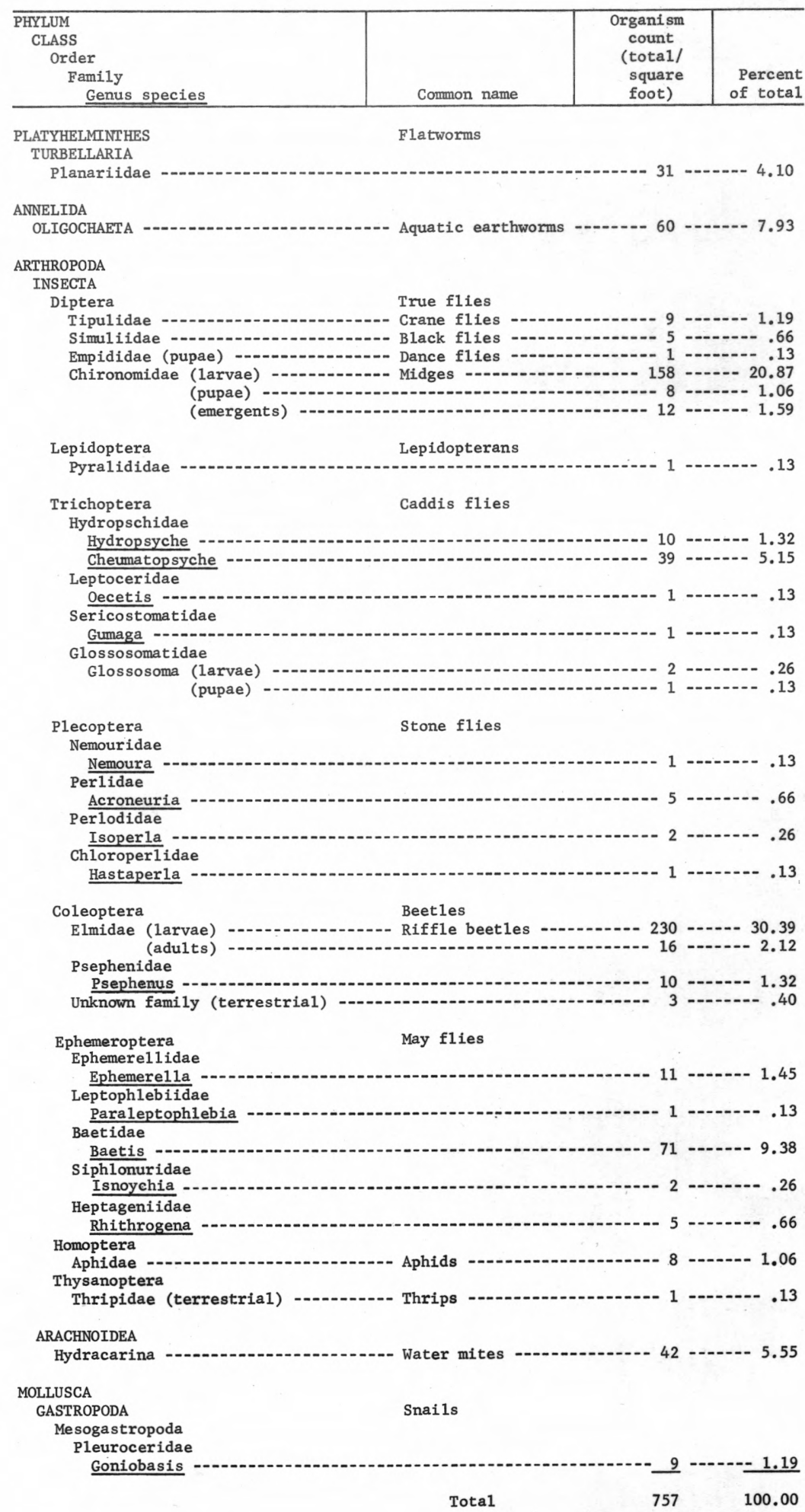


Site number and name: 4 - Lost Creek, Molalla River basin

Date: July 21, 1977

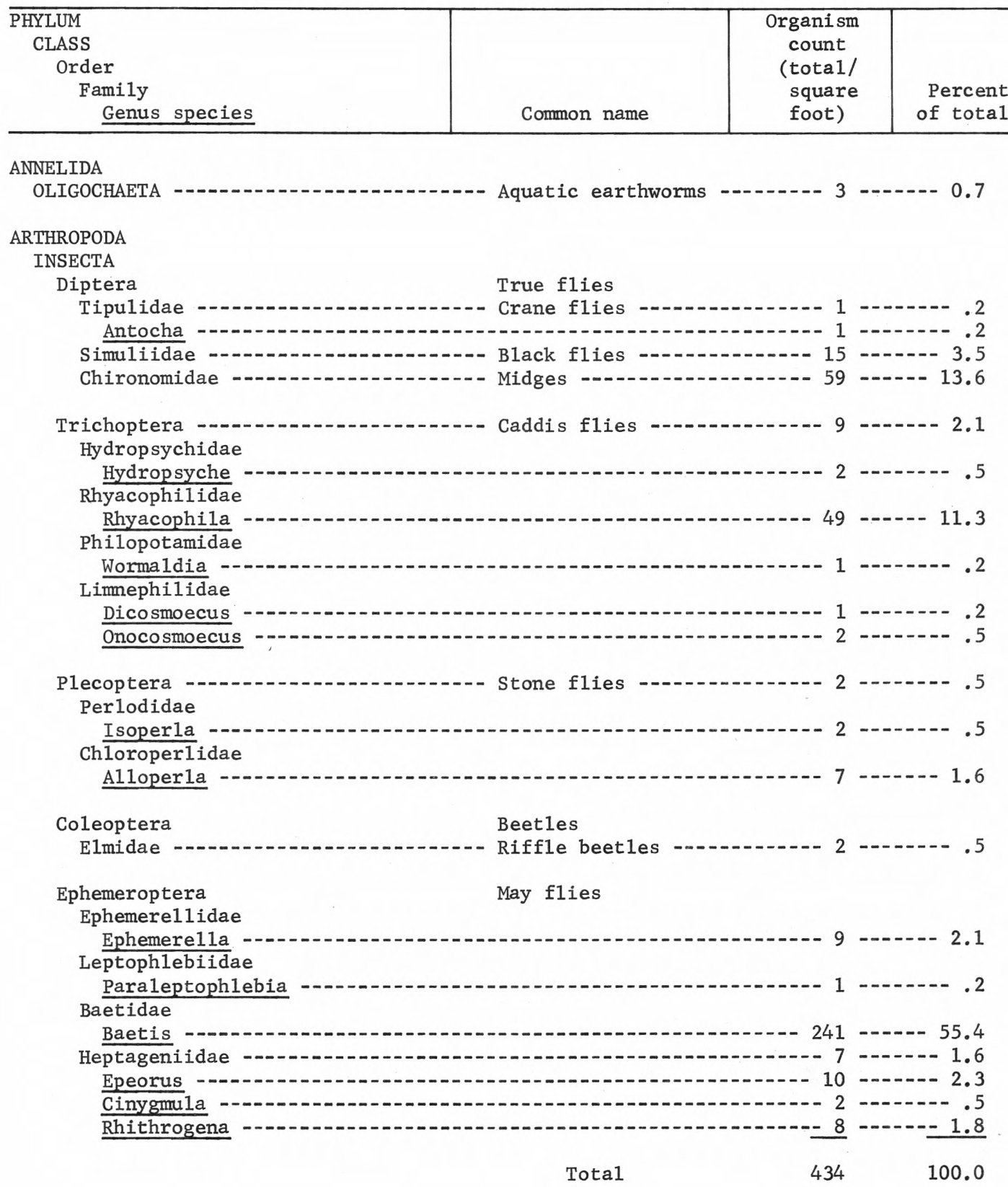


Site number and name: 5 - Dead Horse Canyon Creek, Mola1la River basin

Date: September 6, 1977

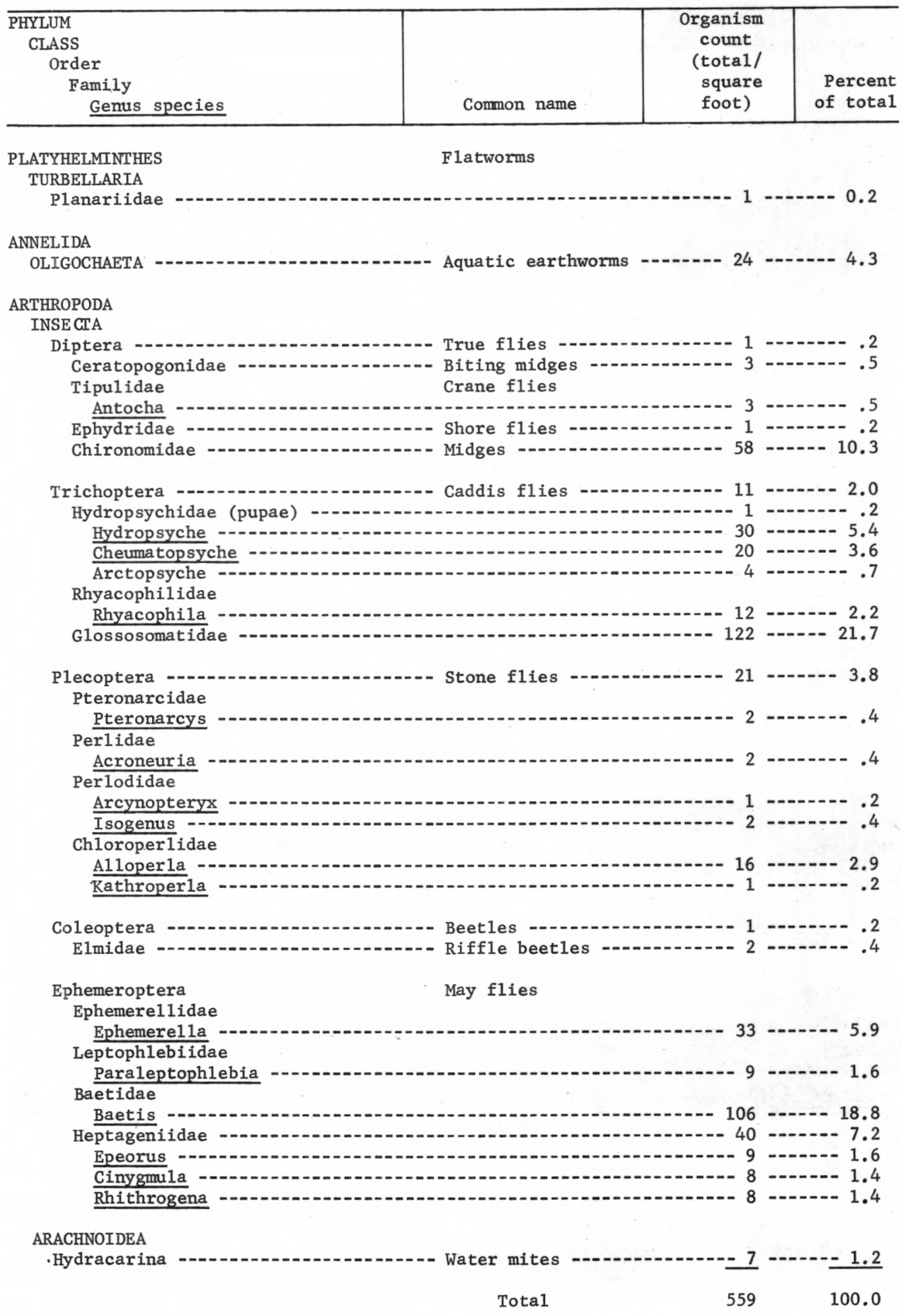


Site number and name: 6 - Dorn Creek, Molalla River basin

Date:

September 14,1977

\begin{tabular}{l|c|c|c}
\hline PHYLUM & & $\begin{array}{c}\text { Organism } \\
\text { count } \\
\text { CLASS }\end{array}$ & $\begin{array}{c}\text { (tota1/ } \\
\text { square }\end{array}$ \\
$\begin{array}{l}\text { Order } \\
\text { Family } \\
\text { Genus species }\end{array}$ & Common name & Percent \\
of total \\
\hline
\end{tabular}

ANNELIDA

OLIGOCHAETA

ARTHROPODA

INSECTA

Diptera - 4 - 4 -

Culicidae - 25 - 25

Simuliidae -

Chironomidae - 213

Trichoptera -

Hydrop sychidae

Hydropsyche - 41 - 3.6

Cheumatopsyche

Rhyacophilidae
Rhyacophila

Philopotamidae

Wormaldia - 124 - 10.7

Glossosomatidae

Plecoptera - 23

Pteronarcidae

Pteronarcella

Peltoperlidae

Peltoperla - 4

Nemouridae

Nemoura - 297 --25.8

Perlodida

Isoper1a - 2

Isogenus - 2 - 2

Chloroperlidae

Alloperla - 29 - 2.5

Hemiptera - 1

Coleoptera -

Elmidae -.....

Ephemeroptera

May f1ies

Leptophlebiidae

Paraleptophlebia - 250

Baetida

Baetis - 30 - 30

Heptageniidae -

Epeorus - 8 - 8

Rhithrogena - 2 - 2

Cinygma -0.-.

MOLLUSCA

GASTROPODA

Snails

Mesogastropoda

P1euroceridae

Goniobasis

$-14---1.2$

Total $1,148 \quad 100$. 
Site number and name: 7 - Milk Creek, Molalla River basin

Date: September 13,1977

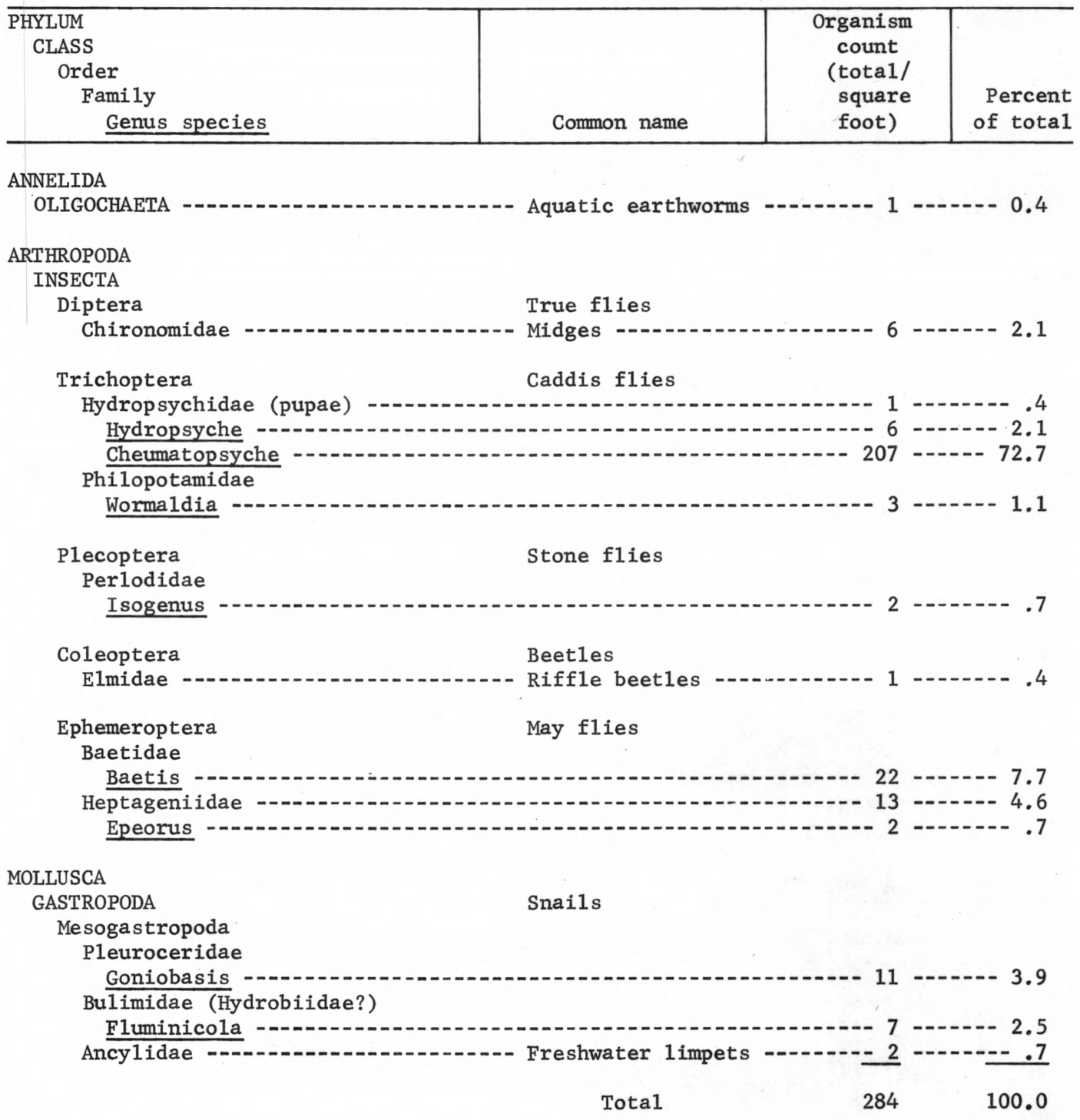


Site number and name: 8 - Wolf Creek, Siuslaw River basin

Date: October 4, 1977

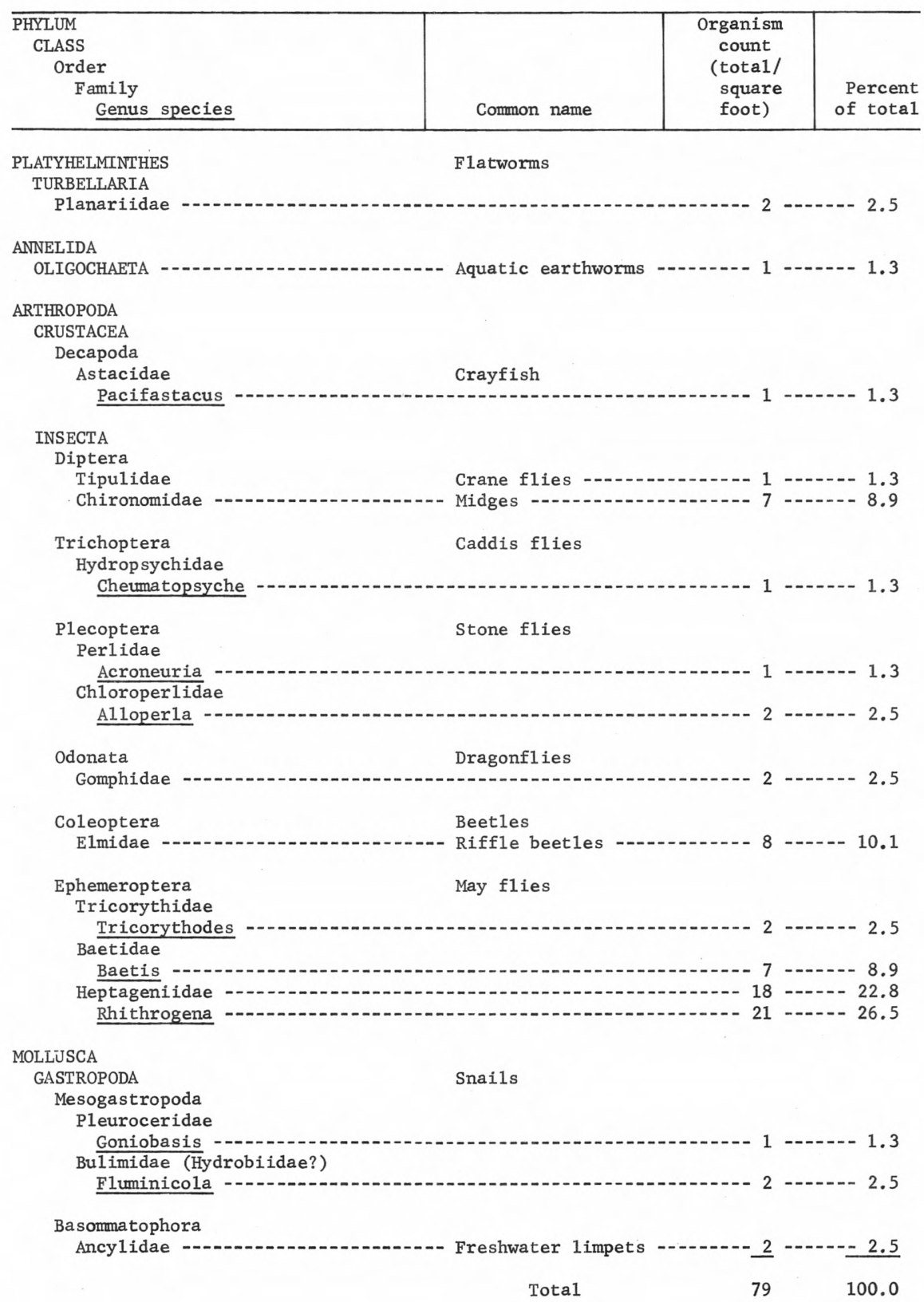


Site number and name: 9 - Knowles Creek, Siuslaw River basin

Date: October 4, 1977

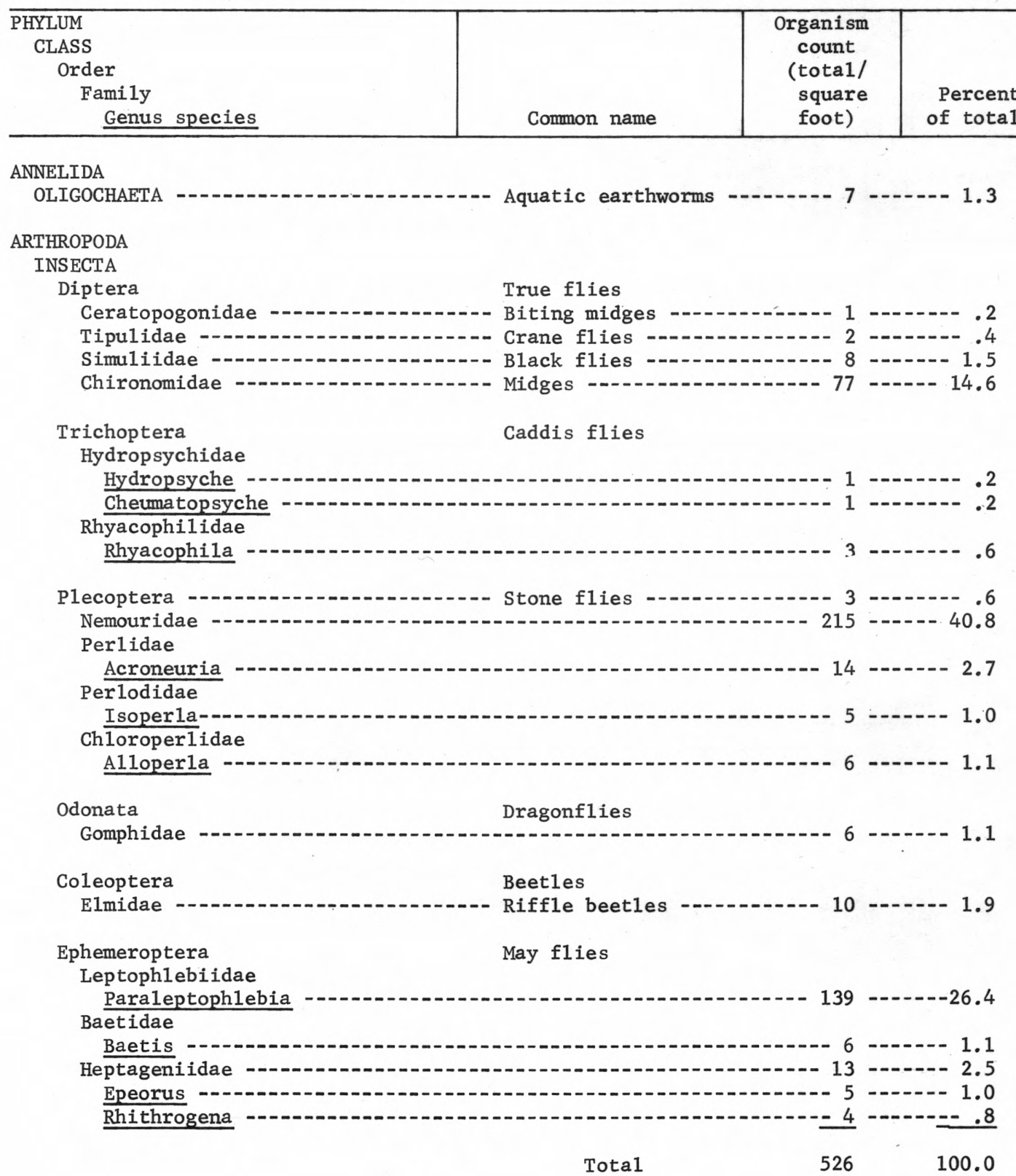


Table 6.--Taxa and numbers of benthic invertebrates--Continued

Site number and name: 10 - Cedar Creek, Siuslaw River basin

Date: August 10, 1977

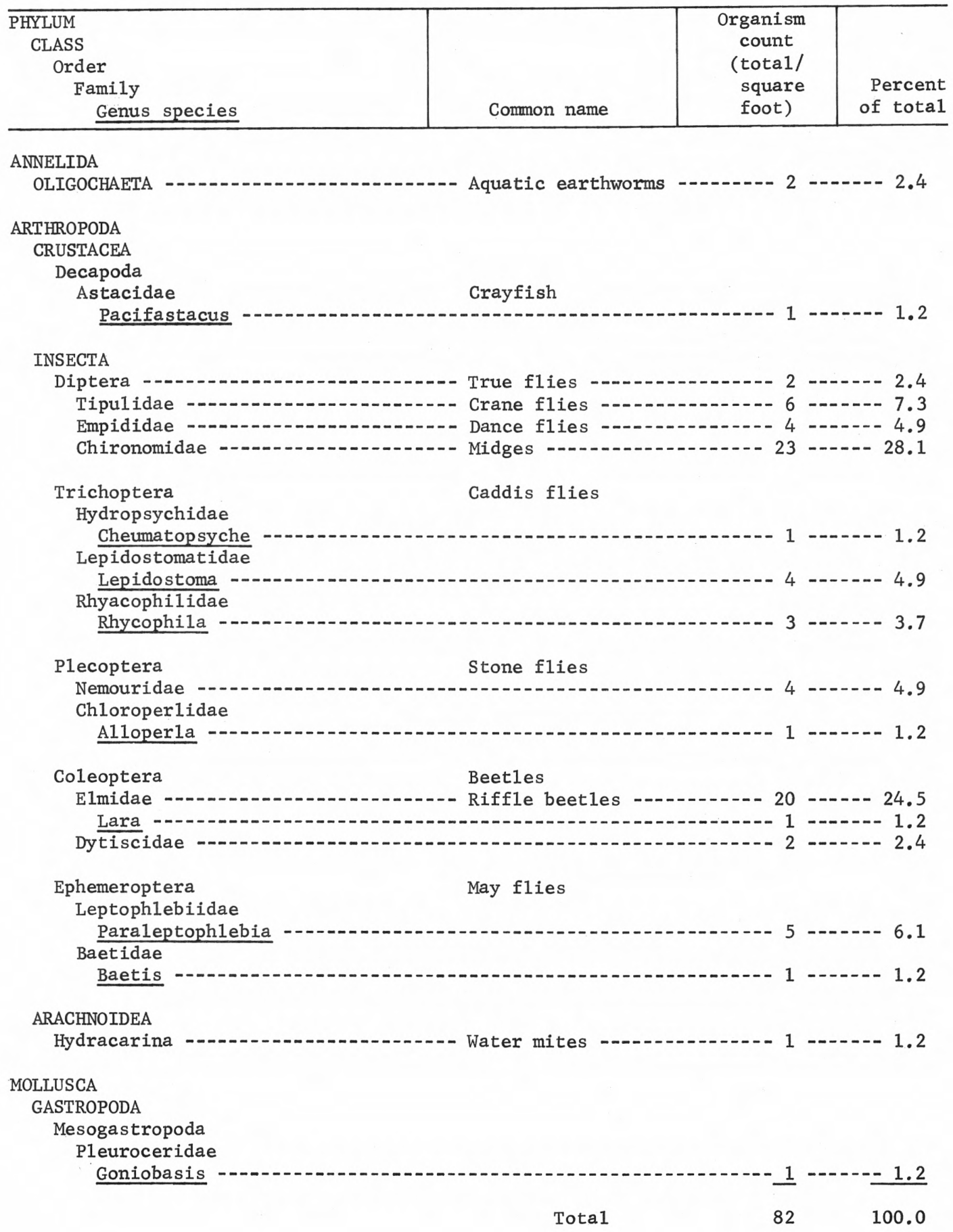


Site number and name: 10-Cedar Creek, Siuslaw River basin

Date: October 5, 1977

\begin{tabular}{|c|c|c|c|}
\hline $\begin{array}{l}\text { PHYLUM } \\
\text { CLASS } \\
\text { Order } \\
\text { Family } \\
\quad \text { Genus species }\end{array}$ & Common name & $\begin{array}{l}\text { Organism } \\
\text { count } \\
\text { (tota1/ } \\
\text { square } \\
\text { foot) }\end{array}$ & $\begin{array}{l}\text { Percent } \\
\text { of tota } 1\end{array}$ \\
\hline \multicolumn{4}{|l|}{ ARTHROPODA } \\
\hline \multicolumn{4}{|l|}{ INSECTA } \\
\hline Diptera & True flies & & \\
\hline Chironomidae & Midges & ---5 & --11.9 \\
\hline $\begin{array}{l}\text { Trichoptera } \\
\text { Hydropsychidae }\end{array}$ & \multicolumn{2}{|c|}{ Hydropsychidae } & \\
\hline \multicolumn{4}{|l|}{ Hydropsyche - } \\
\hline & May flies & Leptophlebiidae & \\
\hline \multicolumn{4}{|l|}{ Baetidae } \\
\hline Baetis & $-2-1-2-1-2$ & $-\ldots-1$ & ---2.4 \\
\hline \multicolumn{4}{|l|}{ Heptageniidae } \\
\hline \multicolumn{4}{|l|}{ MOLLUSCA } \\
\hline $\begin{array}{l}\text { GASTROPODA } \\
\text { Mesogastropoda } \\
\text { Pleuroceridae }\end{array}$ & Snails & & \\
\hline Goniobasis -..- & ----------1 & $\ldots 16$ & -38.1 \\
\hline & Tota1 & 42 & 100.0 \\
\hline
\end{tabular}


Table 6.--Taxa and numbers of benthic invertebrates--Continued

Site number and name: 11 - Bob Creek, Siuslaw Basin

Date: August 9, 1977

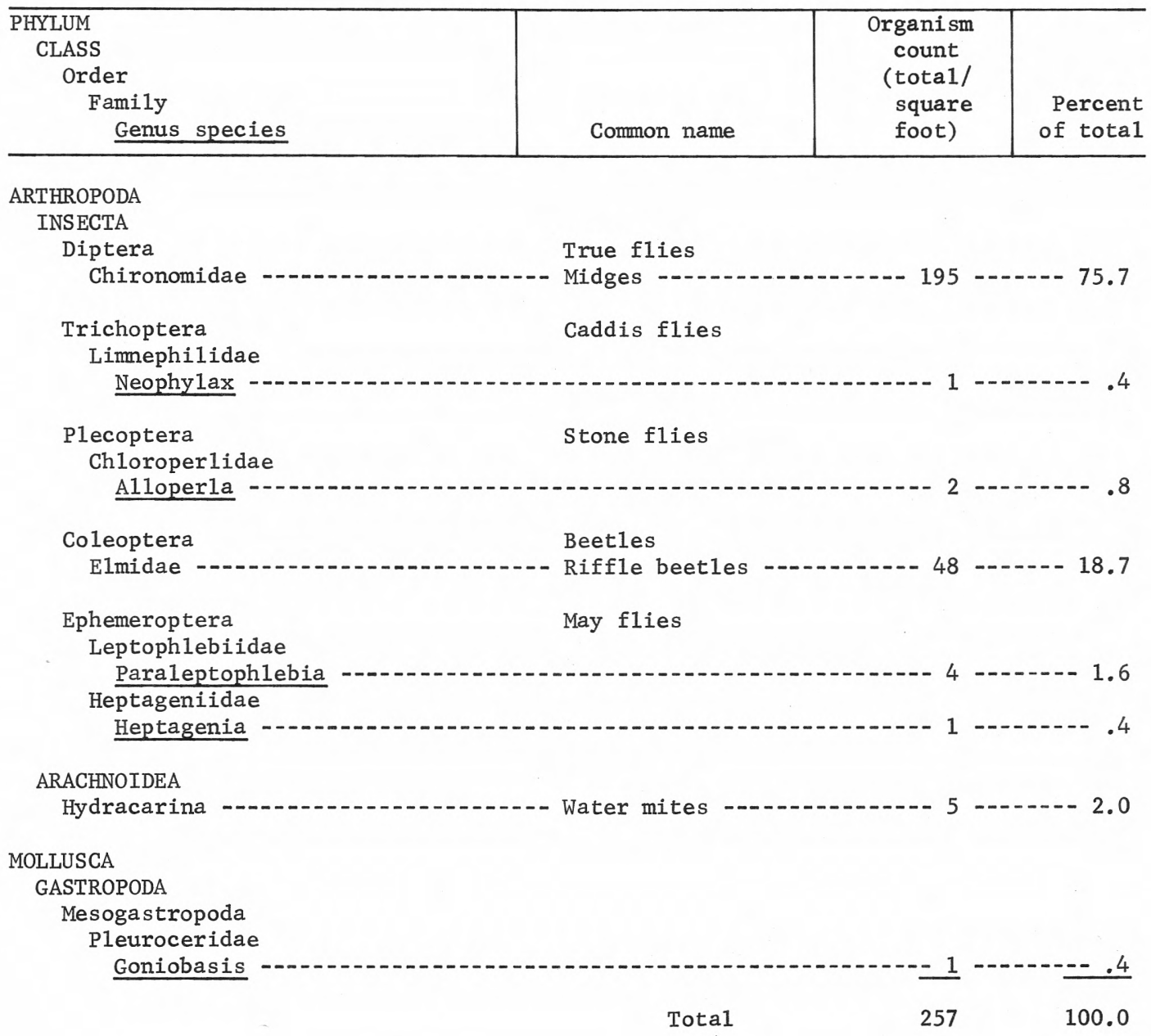


Site number and name: $11-$ Bob Creek, Siuslaw Basin

Date: October 5, 1977

\begin{tabular}{|c|c|c|c|}
\hline $\begin{array}{l}\text { PHYLUM } \\
\text { CLASS } \\
\text { Order } \\
\text { Family } \\
\quad \text { Genus species }\end{array}$ & Common name & $\begin{array}{l}\text { Organism } \\
\text { count } \\
\text { (total/ } \\
\text { square } \\
\text { foot) }\end{array}$ & $\begin{array}{l}\text { Percent } \\
\text { of total }\end{array}$ \\
\hline
\end{tabular}

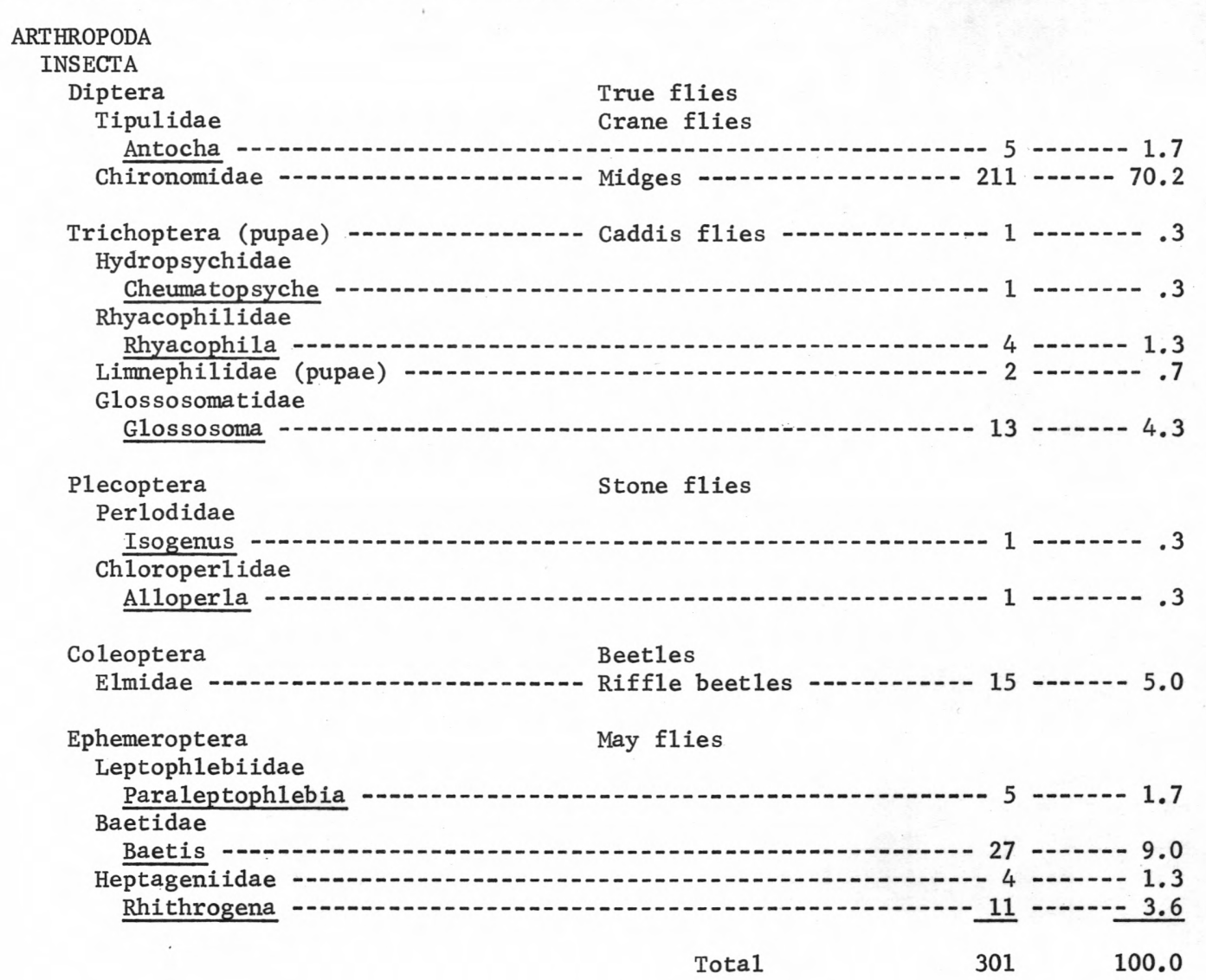




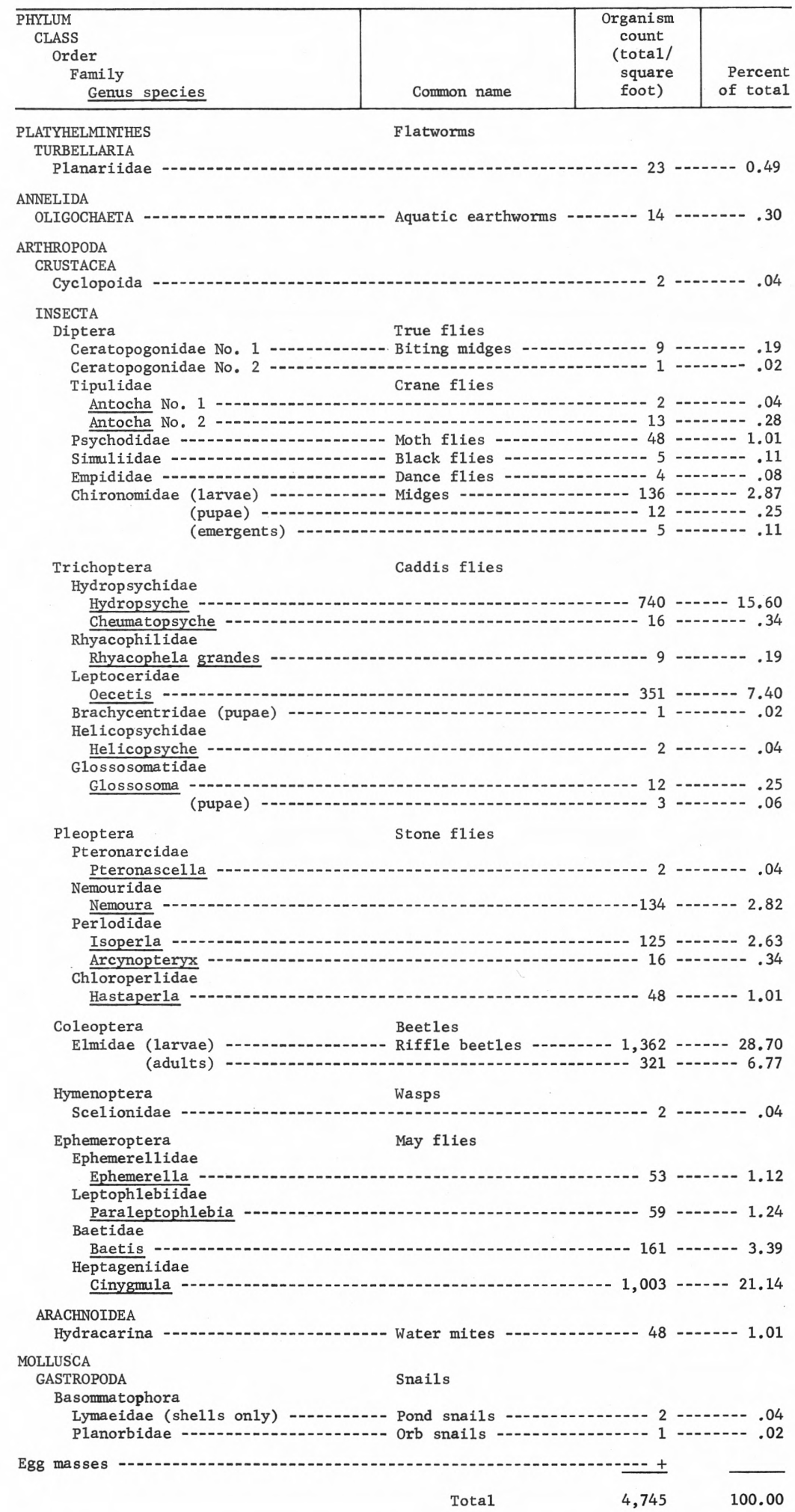


Site number and name: 13 - South Fork John Day River above Little Pine Creek

Date: September 15, 1978

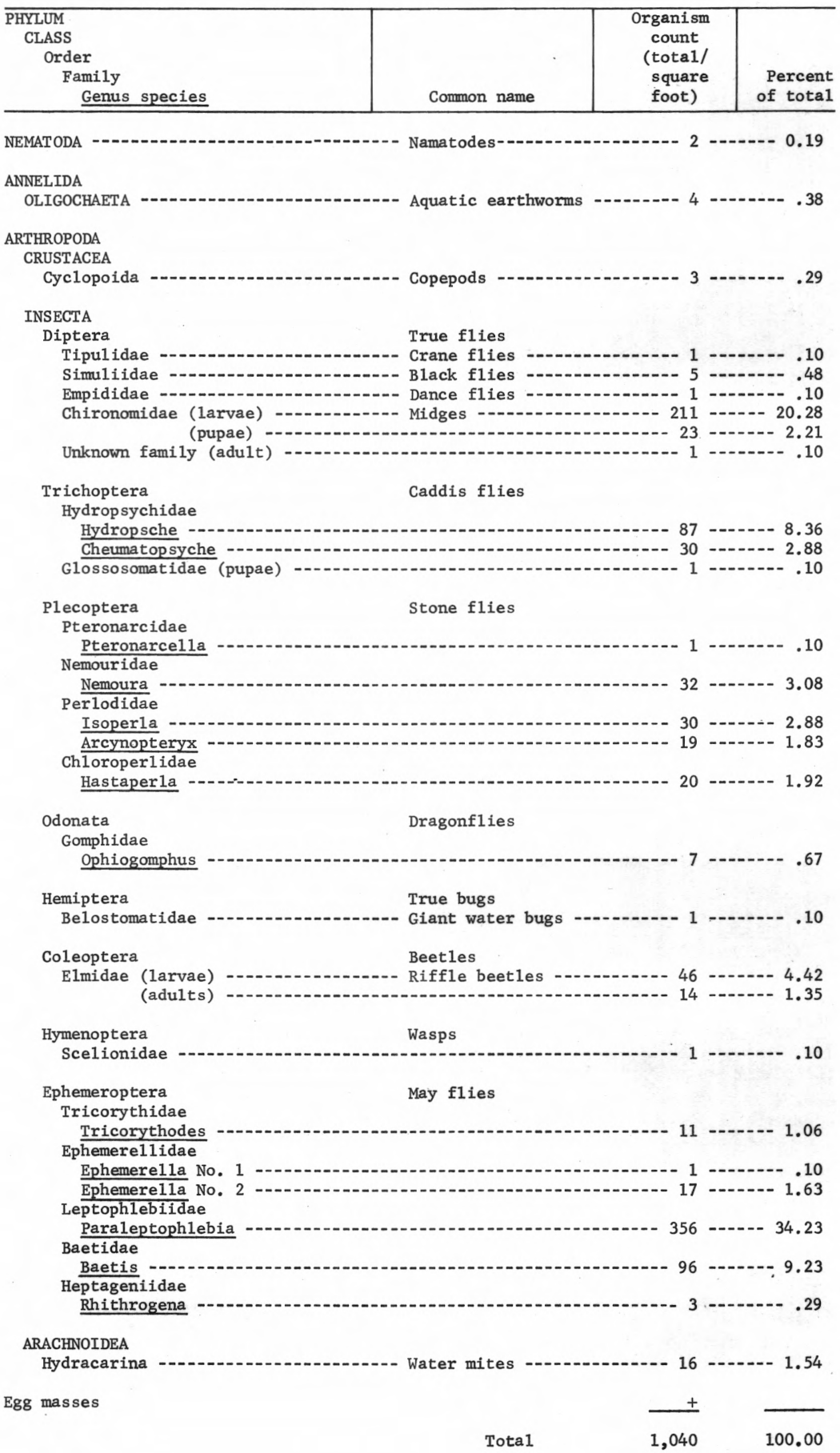


Site number and name: 14 - Black Canyon Creek, South Fork John Day River basin

Date: September 14, 1978

\begin{tabular}{|c|c|c|c|}
\hline $\begin{array}{l}\text { PHYLUM } \\
\text { CLASS } \\
\text { Order } \\
\text { Family } \\
\text { Genus species }\end{array}$ & Common name & $\begin{array}{l}\text { Organism } \\
\text { count } \\
\text { (total/ } \\
\text { square } \\
\text { foot) }\end{array}$ & $\begin{array}{l}\text { Percent } \\
\text { of total }\end{array}$ \\
\hline
\end{tabular}

ANNELIDA

OLIGOCHAETA

ARTHROPODA

CRUSTACE

Decapoda

Astacidae

Pacifactacus - 04

INSECTA

Diptera

Ceratopogonidae -

Tipulidae

Antocha - Crane flies

Tipulidae (pupae) - No

Psychodidae - .

Simuliidae -... Black flies -...... 49 -... 2.01

Tabanidae -.- Horse flies -

Empididae - -

Chironomidae (larvae) (pupae)

Trichoptera Caddis flies

Hydropsychidae

Hydropsyche - 321

Rhyacophilida

Rhyacophia -

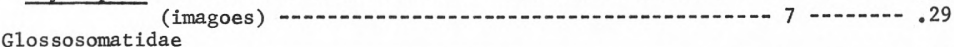

Glossosoma - 64 - 62

Plecoptera

Stone flies

Pteronaryidae

Pteronarella -

Nemouridae

Nemoura -

Perlidae

Acroneura (Hesperoperla)

Perlodidae

Isoperla -

Arcynopteryx -...... 29

Chloroperlidae

Hastaper1a

Megalopterans (pupae) - 1 - 1 - 04

Odonata

Dragonf1ies

Gomphidae

Ophiogomphus

Coleoptera

Elmidae (larvae)

(adu1ts) -

Ephemeroptera

Tricorythida

May flies

Tricorythodes

Ephemerella

Baetidae

Baetis - 293 - 12.00

Leptoph1ebiidae

Paraleptoph1ebia - 2.83

Heptogeniidae

Epeorus -

Rithrogena -

ARACHNOIDEA

Hydracarina - 34

MOLLUSCA

BIVALVIA

Nuculoidea

Sphaeriidae

Fingernail clams

Pisidium -

Total $2,441 \quad 100.00$ 
Site number and name 15 - South Fork John Day River at Dayville

Date: September 7, 1977

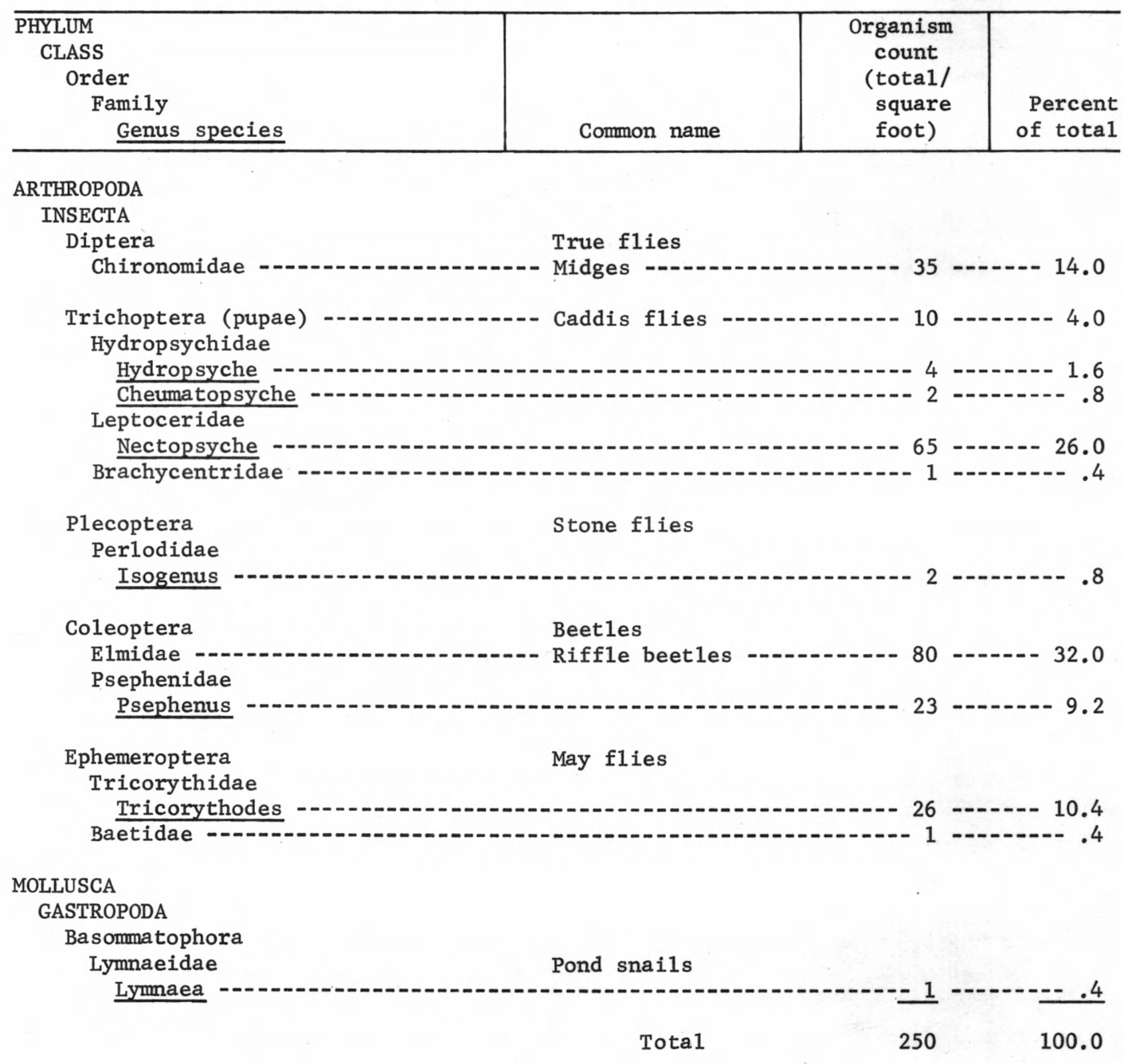


Site number and name: 15 - South Fork John Day River at Dayville

Date: September 13,1978

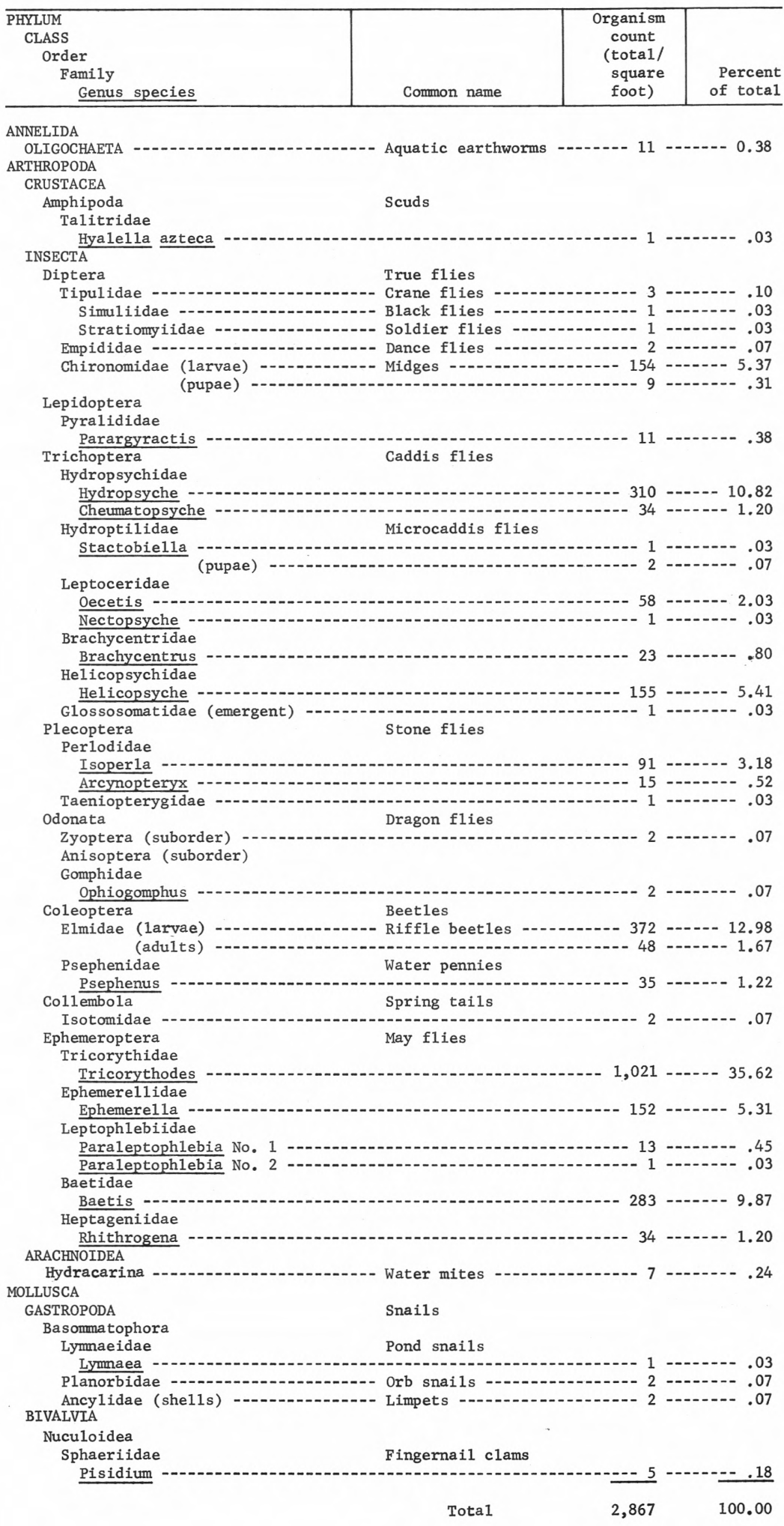


Site number and name: 16 - Pearson Creek, Umatilla River basin

Date: September 20, 1978

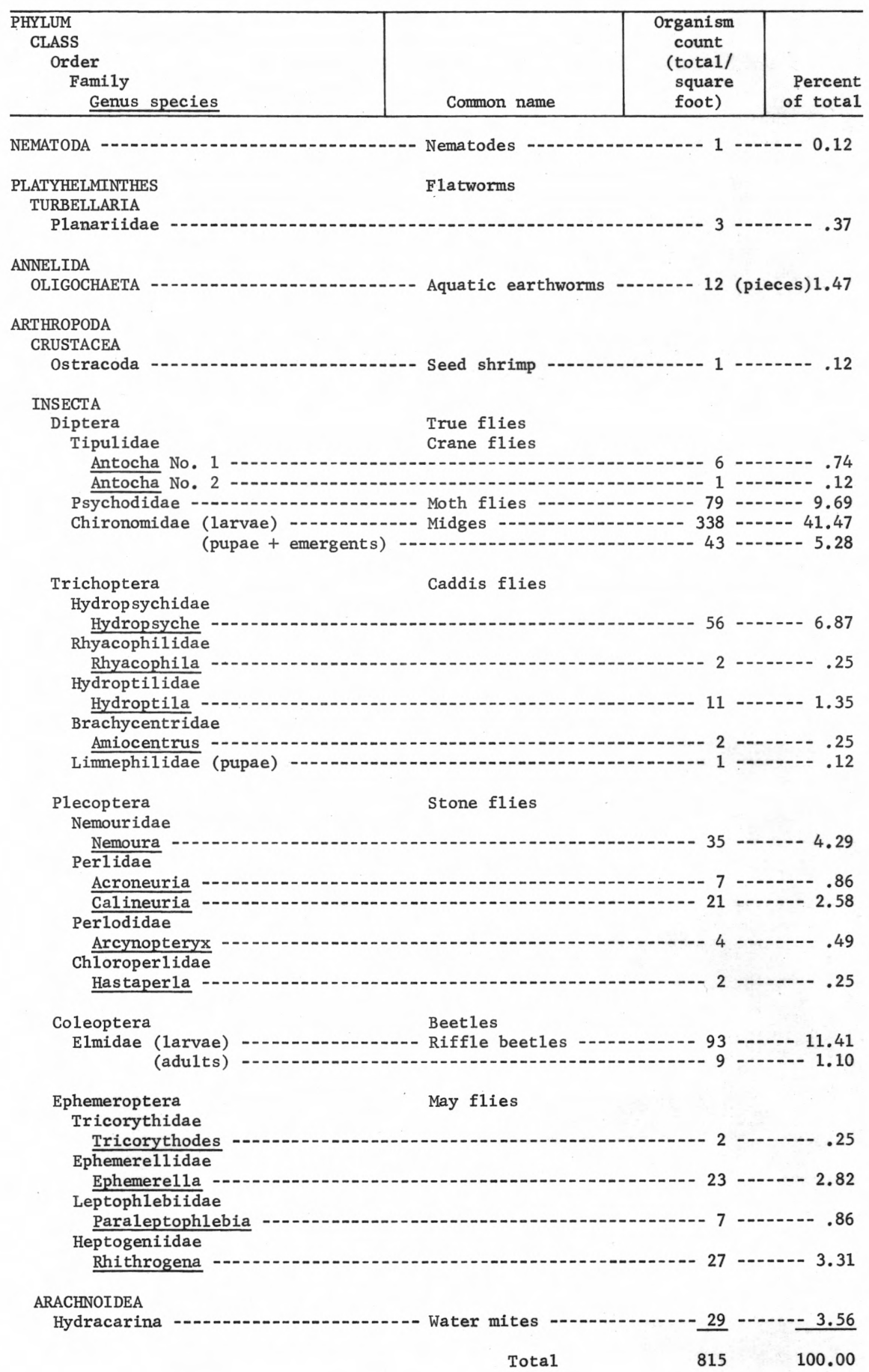


Site number and name: 17 - East Fork Birch Creek, Umatilla River basin

Date: September 22, 1978

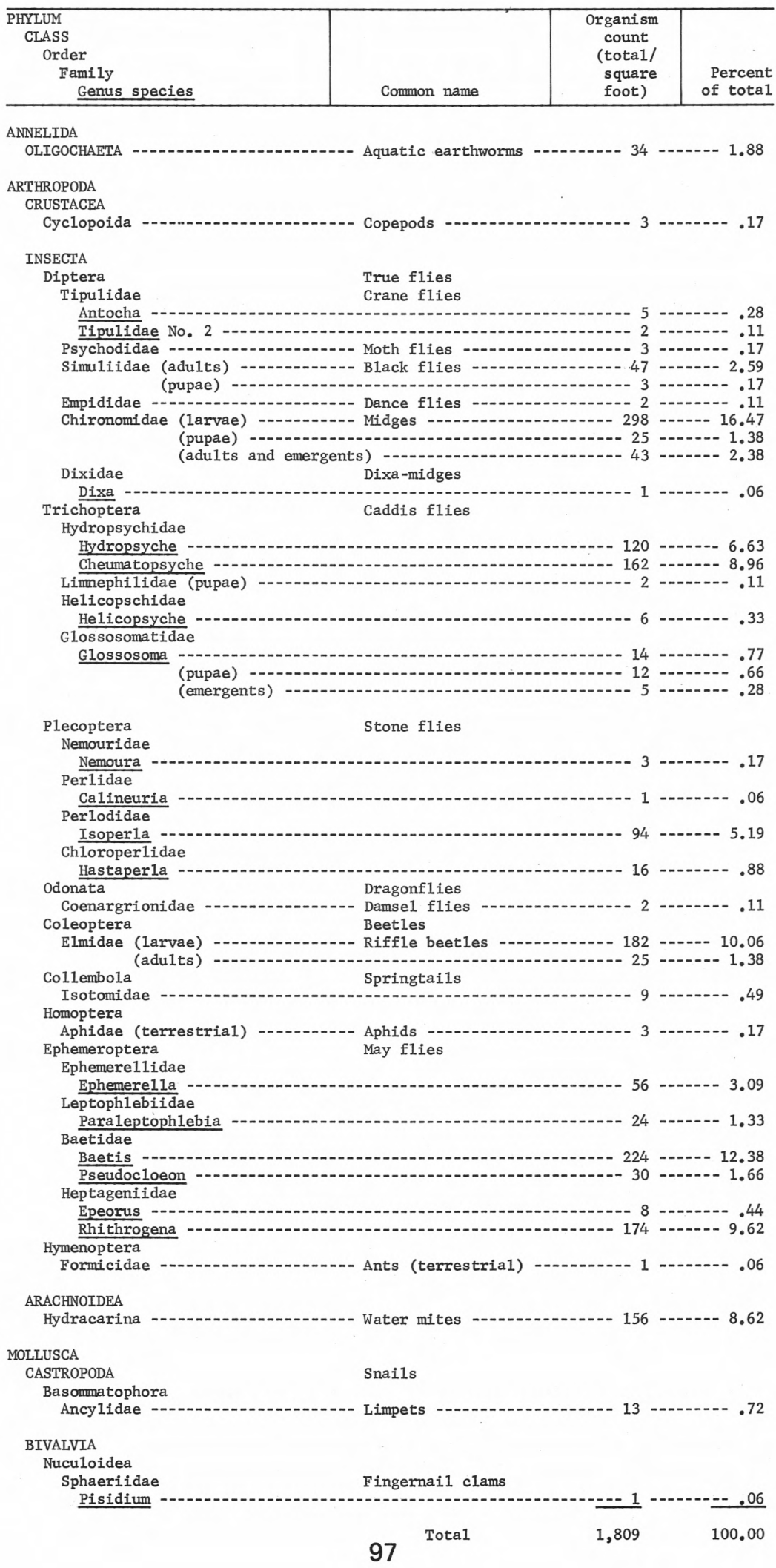


Site number and name: 18 - Wildhorse Creek, Umatilla River basin

Date: September 22, 1978

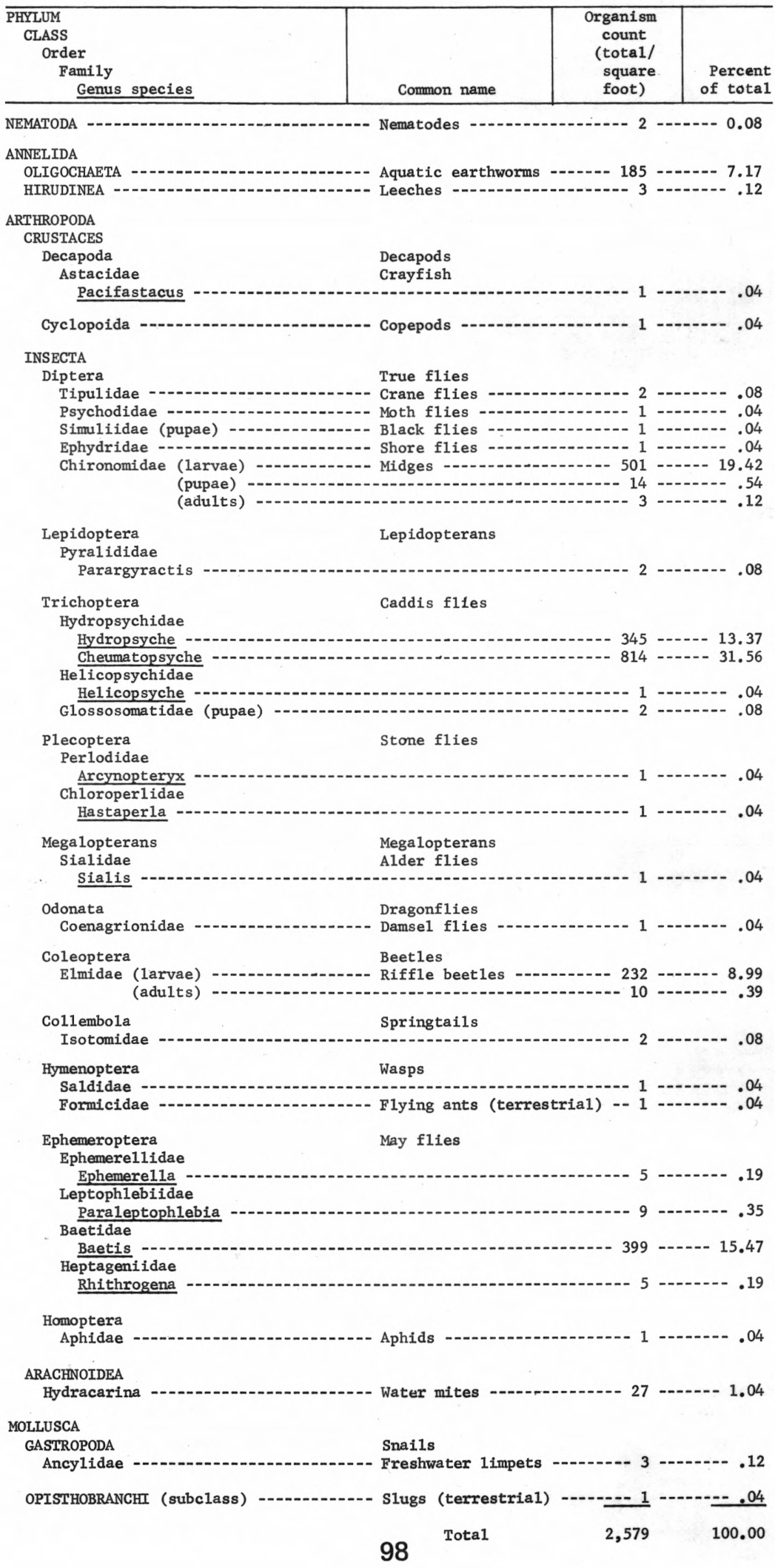


